\title{
Mathematical Modeling of Transpiration Cooling in Cylindrical Domain
}

\section{Mehdi Moghadasi Faridani}

\author{
Submitted to the \\ Institute of Graduate Studies and Research \\ in partial fulfilment of the requirements for the Degree of
}

\author{
Doctor of Philosophy \\ in \\ Mechanical Engineering
}

Eastern Mediterranean University

January 2015

Gazimağusa, North Cyprus 
Approval of the Institute of Graduate Studies and Research

Prof. Dr. Elvan Yilmaz

Director

I certify that this thesis satisfies the requirements as a thesis for the degree of Doctor of Philosophy in Mechanical Engineering.

Prof. Dr. Uğur Atikol

Chair, Department of Mechanical Engineering

We certify that we have read this thesis and that in our opinion it is fully adequate in scope and quality as a thesis for the degree of Doctor of Philosophy in Mechanical Engineering.

Prof. Dr. Hikmet Ş. Aybar

Supervisor

Examining Committee

1. Prof. Dr. Tahir Yavuz

2. Prof. Dr. Hikmet Ş. Aybar

3. Prof. Dr. Şenol Başkaya

4. Prof. Dr. İbrahim Sezai

5. Assoc. Prof. Dr. Hasan Hacışevki 


\begin{abstract}
This research is conducted to develop numerical schemes modeling one-dimensional steady-state non-equilibrium transpiration cooling in Si-C porous materials. The transpiration cooling process is assumed to be used to protect the thrust chamber of a liquid rocket engine by blocking the enormous heat transfer towards its walls. The flow modeling within the porous media is performed through applying numerical schemes to the compressible flow of the coolant in cylindrical coordinates. The results of this modeling are validated by the experimental results obtained from a study in the University of Stuttgart. Also, a numerical method is developed to solve the equations of hot gases which are flowing through the nozzle in order to provide the hot boundary condition of the porous segment. The flow equations within the porous medium are solved separately, to analyze the important parameters affecting the transpiration cooling process. The effect of coolant mass flow rate, hot face boundary condition type, gas type, and sample length are observed as well. Moreover, the internal convective heat transfer between solid structure and the coolant flow is studied. The porous solver code is then coupled with the gas solver code so that the cooling process can be studied in a more realistic area. The results show the variation of gas properties through the nozzle the effect of transpiration cooling on them. Also, a sample method of controlling the nozzle wall temperature is demonstrated.
\end{abstract}

Keywords: Transpiration cooling, cylindrical, porous media, thrust chamber 


\section{ÖZ}

$\mathrm{Bu}$ araştırma Si-C gözenekli malzemelerde tek boyutlu, kararlı durumda, denge dışı terleme soğutmanın sayısal şema modellemesinin geliştirilmesi için yapılmıştır. Terleme soğutma işlemi sıvı roket motorunun itme odasını korumak için odanın duvarlarına karşı muazzam 1sı transferini engeller. Gözenekli ortam içinde akış modelleme, soğutucunun sıkıştırılabilir akışı silindirik eşgüdüm içinde sayısal düzenler uygulanarak gerçekleştirilir. $\mathrm{Bu}$ modellemenin sonuçları Stuttgart Üniversitesi'nde yürütülen bir çalışmadan elde edilen deneysel sonuçlar tarafından doğrulanmaktadır. Ayrıca, gözenekli parçanın sıcak sınır koşulunu sağlamak için ağızlık boyunca akan sıcak gazların denklemlerini çözmek için sayısal yöntem geliştirilmiştir. Terleme soğutma sürecini etkileyen önemli parametreleri analiz etmek için gözenekli ortamda akış denklemleri ayrı ayrı çözülmüştür. Soğutucu kütle akış oranının etkisi olarak sıcak yüz sınır koşulu türü, gaz türü ve numune uzunluğu gözlenmektedir. Ayrıca, katı yapı ve soğutucu akış arasındaki iç konvektif ısı transferi incelenmiştir. Gözenekli çözücü kod daha sonra gaz çözücü kod ile birleştirilir, böylece soğutma işlemi daha gerçekçi bir alanda incelenebilir. Terleme soğutma etkisini ağızlık boyunca gaz özelliklerini degiştirerek gösterir. Ayrıca, ağızlık duvar sıcaklığının kontrol edilmesi için örnek yöntem gösterilmiştir.

AnahtarKelimeler: terleme soğutma, silindirik, gözenekli malzemeler, itme odası. 
To My Family 


\section{ACKNOWLEDGMENT}

First, I would like to thank Prof. Dr. Hikmet Ş. Aybar and Prof. Dr. Mehmet Sozen who have supported me through my thesis and for their excellent guidance and knowledge. Without their invaluable supervision, it would have been impossible for me to complete this work. Also I would like to thank Prof. Dr. Ugur Atikol for his helps and supports during my $\mathrm{PhD}$ program.

Finally, I thank my parents, family, and my wife for their unconditional support, both financially and emotionally through my degree. 


\section{TABLE OF CONTENT}

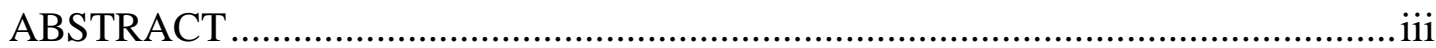

ÖZ

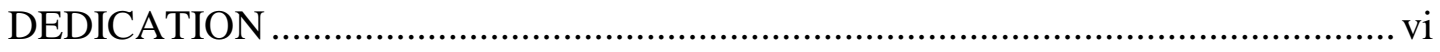

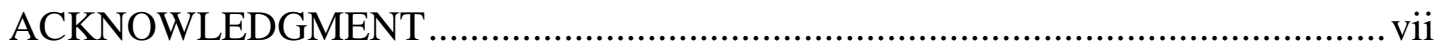

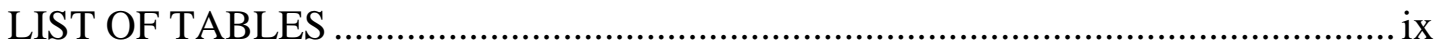

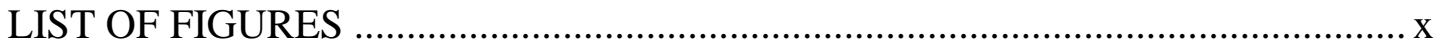

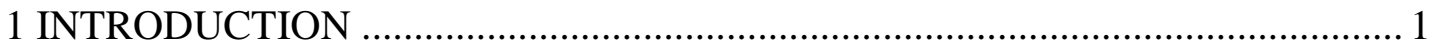

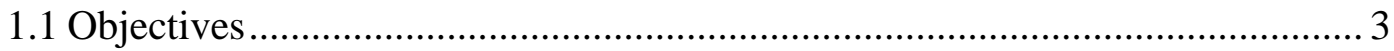

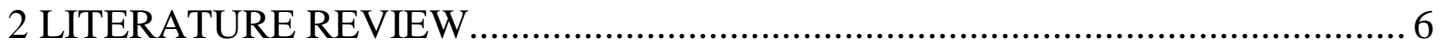

3 POROUS MEDIA: SYSTEM DESCRIPTION \& EQUATIONS ......................... 12

3.1 Mathematical Modeling in Cylindrical System ............................................ 14

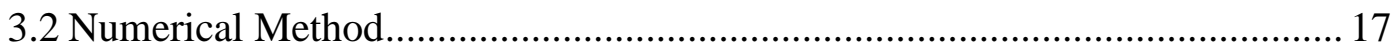

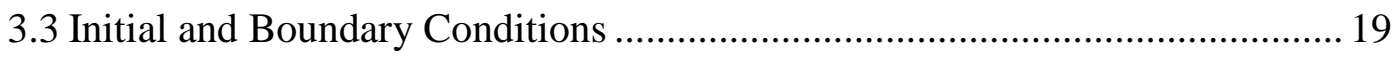

3.4 Mathematical Modeling in Cartesian System and Benchmarking .................. 22

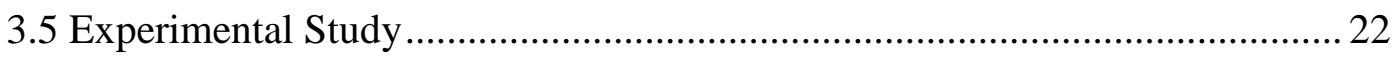

3.6 Comparison of Experimental and Numerical Results .................................. 24

4 THRUST CHAMBER: SYSTEM DESCRIPTION \& EQUATIONS..................... 27

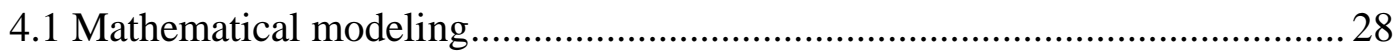

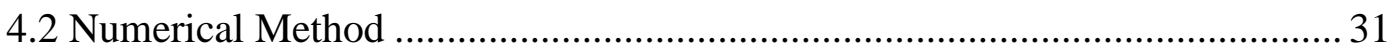


4.3 Transonic Region

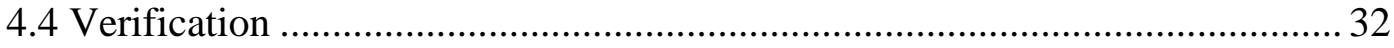

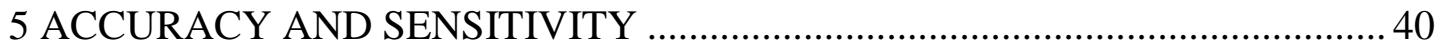

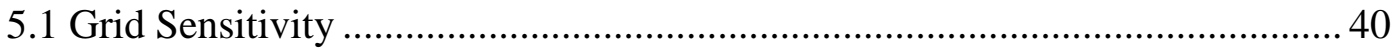

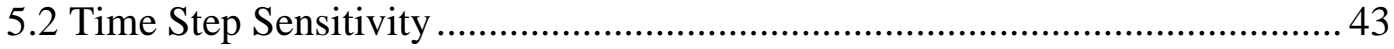

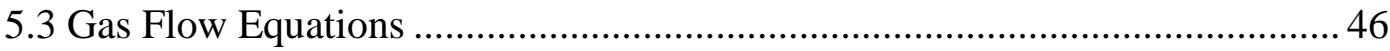

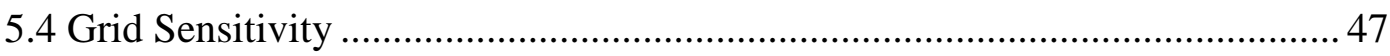

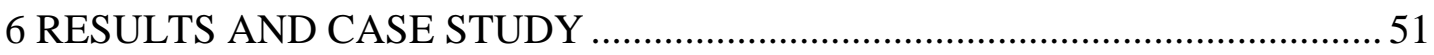

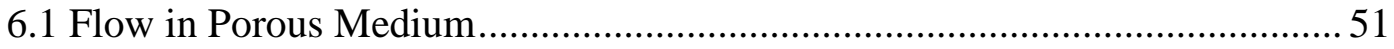

6.2 Effect of Coolant Mass Flow Rate................................................................ 52

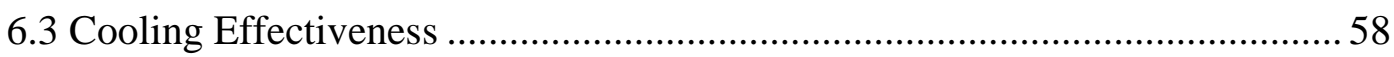

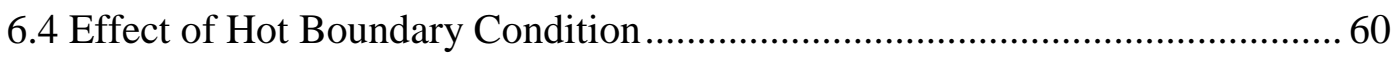

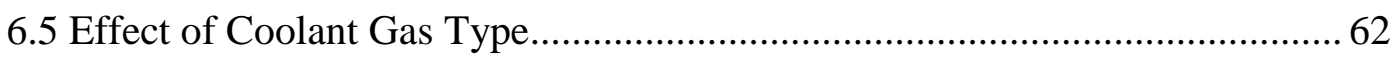

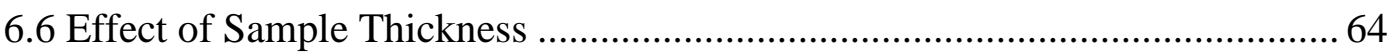

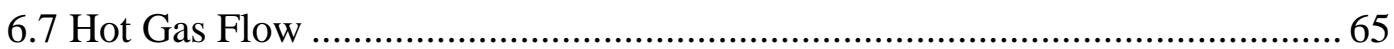

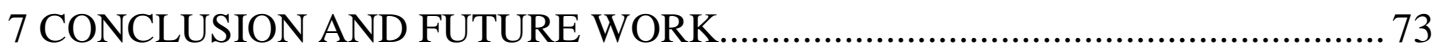

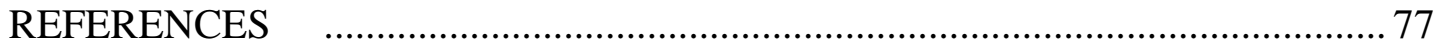




\section{LIST OF TABLES}

Table 1: Investigated Sample PH1606-1 [15]

Table 2: Different mass flow rates and corresponding boundary temperatures [15]. 24

Table 3: Relative error analysis of hot face temperature for explicit method for grid sensitivity error $=\mathbf{T}-\mathbf{T} 31 \mathrm{~T} 31 \times 100$

Table 4: Relative error analysis of hot face temperature for Implicit Method for grid sensitivity error $=\mathbf{T}-\mathbf{T} 31 \mathrm{~T} 31 \times \mathbf{1 0 0}$

Table 5: Running time for each model (in seconds) for different time steps using 21 nodes. 46

Table 6: Mach number values at the throat and the relative error error $=\mathbf{M t h}-$ Mth, 201Mth, $201 \times 100$ 50

Table 7: Properties of Si-C porous material 52 


\section{LIST OF FIGURES}

Figure 1: Schematic of transpiration cooling process.

Figure 2: Schematic illustration of transpiration cooled thrust chamber of a rocket. 13

Figure 3: Schematic of porous layer and its inner/outer surfaces. 14

Figure 4: Temperature profile within the domain. 16

Figure 5: Grid points within the porous medium, internal and interpolated nodes. .. 18

Figure 6: Experimental Setup. 23

Figure 7: Temperature profile of the numerical solution of compressible flow in the Cartesian system (solid lines) compared with the experimental results (symbols) considering different Air mass flow rates. 25

Figure 8: Profile of the converging-diverging nozzle. 28

Figure 9: An infinitesimal control volume within the nozzle. 29

Figure 10: Non-dimensional values of Mach number, temperature, and pressure for isentropic flow along the nozzle.

Figure 11: Mach number distribution of a flow with friction in an adiabatic constant area duct. 37

Figure 12: Mach number distribution of a flow with heat addition in a frictionless constant area duct. 39

Figure 13: Temperature profile and grid sensitivity of explicit scheme for different nodes of $6,11,21$, and 31 and $\mathrm{dt}=0.1$.

Figure 14: Temperature profile and grid sensitivity of implicit scheme for different nodes of $6,11,21$, and 31 and $\mathrm{dt}=0.1$. 42 Figure 15: Temperature profile and time step sensitivity of explicit scheme for different time steps of 5.0, 1.0, 0.2, 0.04, 0.008 and 0.0016 and 21 nodes. 44 
Figure 16: Temperature profile and time step sensitivity of implicit scheme model for different time steps of 5.0, 1.0, 0.2, 0.04, 0.008 and 0.0016 and 21 nodes 45

Figure 17: Mach number profile and grid sensitivity for different nodes of 31, 41, 51, 81, 121, and 201.

Figure 18: Mach number profile at the throat (i.e. $\mathrm{x}=0.5 \mathrm{~m}$ ) for different nodes of 31 , 41, 51, 81, 121, and 201. 49

Figure 19: Temperature profile for different mass flow rates within the sample. ..... 53 Figure 20: Temperature profile at the hot and cold faces for different mass flow rates.

Figure 21: Radial velocity profile the coolant flow towards the hot face for different coolant flow rates within the sample. 55

Figure 22: Density profile of the coolant flow for different coolant flow rates within the sample 57

Figure 23: Heat flux distribution within the porous sample for different coolant mass flow rates. 58

Figure 24: Effectiveness Factor for different mass flow rates. 60

Figure 25: Effect of hot face heat transfer coefficient on temperature profile within the sample

Figure 26: Effect of hot gas temperature on temperature profile within the sample. 62 Figure 27: Temperature profile within the sample for different gases: Air, Helium, and Hydrogen.

Figure 28: Temperature profile within the sample for different sample lengths of 6,8 , 12, 16, and $20 \mathrm{~cm}$. 64

Figure 29: Porous one-d segments covering the converging part of nozzle. 66

Figure 30: Mach number profile within the nozzle for initial and final solutions. .... 68 
Figure 31: Gas temperature profile within the nozzle for initial and final solutions. 69 Figure 32: Coefficient of convective heat transfer at the wall for the final solution. 70 Figure 33: Convective heat transfer profile between the hot gases and the porous wall within the nozzle. 71

Figure 34: Wall temperature distribution within the nozzle for the initial and final solutions. 72 


\section{LIST OF NOMENCLATURE}

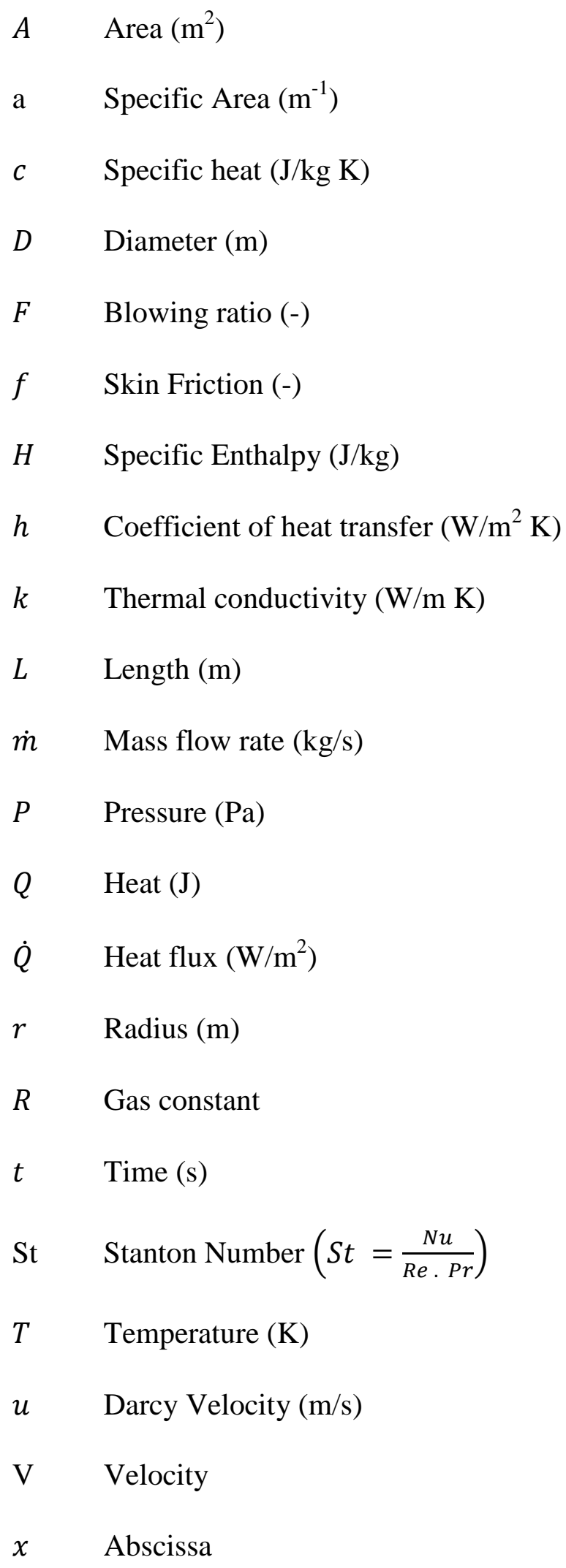




$\begin{array}{ll}\alpha & \text { Permeability coefficient } \\ \alpha^{\prime} & \text { Thermal diffusivity } \\ \beta & \text { Permeability coefficient } \\ \varepsilon & \text { Porosity } \\ \epsilon & \text { Effectiveness } \\ \lambda & \text { Eigen value } \\ \mu & \text { Viscosity }\left(\mathrm{N} \cdot \mathrm{s} / \mathrm{m}^{2}\right) \\ \rho & \text { Density }\left(\mathrm{kg} / \mathrm{m}^{3}\right)\end{array}$

\section{Subscripts}

$\begin{array}{ll}A & \text { Average } \\ c & \text { Coolant } \\ \text { d } & \text { Difference } \\ f & \text { Fluid } \\ h & \text { Hot } \\ i & \text { Inner } \\ o & \text { Outer } \\ s & \text { Solid } \\ t & \text { Stagnation (total) } \\ w & \text { Wall } \\ h g & \text { Hot Gas } \\ e f f & \text { Effective } \\ \text { init } & \text { Initial }\end{array}$

Abbreviations

Si $/ C$ Silicon - Carbon 
C $C$ Carbon - Carbon 


\section{Chapter 1}

\section{INTRODUCTION}

One of the most important issues of designing and running space vehicles is thermal protection system (TPS), specifically, cooling technologies. Major cooling processes are necessary to be applied to re-entry vehicles and rocket propulsion systems including thrust chamber and nozzle. The cooling technologies become influential as erosion and ablation may occur to their walls. Ablation can be described as a surface recession process taking place on the wall of a nozzle because of high velocity and high temperature gases passing over the surface. For instance, the nozzle throat in a rocket engine is designed such that it produces a certain thrust force and its cross sectional area has to be maintained at a standard range. Thus, it needs to be cooled and protected against ablation, specially, in case of reusing the rocket in next missions. In addition, since in a liquid rocket engine, the combustion temperature is almost twice the melting point of steel, this thermal load may cause the rocket wall temperature exceed its allowable limit at which it could not carry its loads. Transpiration cooling is the process of injecting a coolant fluid into a porous layered material in order to block enormous incoming heat flux towards the main structure. It is mostly used when the regenerative and film cooling are not sufficient. The main purpose of this process is to absorb a significant amount of heat and by coolant and transport it to the thrust chamber. 
The schematic of transpiration cooling process is illustrated in Fig.1. Exhaust gases of the rocket engine are flowing through the thrust chamber and has the Mach number, temperature, and pressure of $\mathrm{M}_{\mathrm{g}}, \mathrm{T}_{\mathrm{g}}$, and $\mathrm{P}_{\mathrm{g}}$, respectively. The major heat transfer mechanisms towards the chamber wall are convection and radiation, where the convective term dominates the radiative term. The convective heat flux to the wall can be expressed in terms of Stanton number $\left(S t=\frac{N u}{R e . P r}\right)$ which is a function of Nusselt, Reynolds, and Prandtl numbers. The main gas flow creates a boundary layer over the wall within the thrust chamber. Subsequently, the coolant at a mass flow rate of $\dot{m}_{c}$ is injected through the porous medium flowing towards the thrust chamber boundary layer. The coolant, in other words, blocks the heat flux incoming from the boundary layer by absorbing and flowing towards it. This process is similar to what takes place in a counter flow heat exchanger. The heat exchange between porous structure and the coolant occurs within the tiny pores of the porous material. Thus, it can be a function of some parameters of both solid and fluid, such as the coolant mass flow rate and the porosity of the segment. For higher ranges of this heat exchange, a thermal equilibrium between the local wall temperature and the local coolant temperature can be an appropriate assumption. As shown in Fig1.1, the coolant affects the boundary layer as it penetrates the main flow and due to that, the boundary layer thickness increases. More precisely, the effect of transpiration cooling on the main flow can be expressed as reducing the temperature and velocity gradients at the cooled wall. This, means the lower heat transfer and friction to the thrust chamber wall from the main flow. 


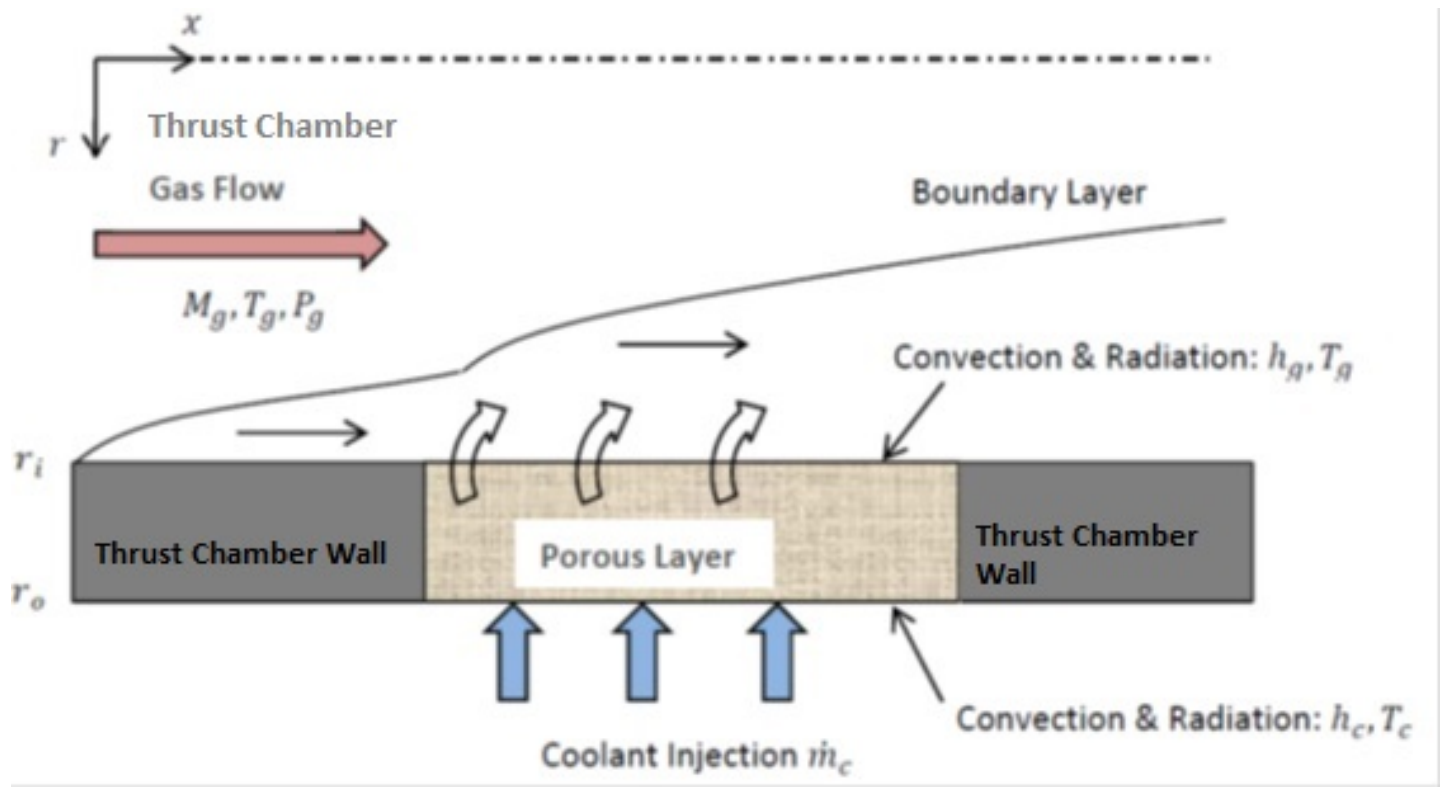

Figure 1: Schematic of transpiration cooling process.

\subsection{Objectives}

As mentioned earlier, further detailed and parametric studies are necessary to demonstrate the functionality and applicability of transpiration cooling in a rocket engine. A comprehensive one-dimensional mathematical modeling is proposed to simulate this cooling technique and its corresponding details. This model should be capable of covering the lacking points of the previous studies besides yielding reliable results. The presented model contains a numerical method of flow simulation in porous media in cylindrical coordinates which is coupled with a one-dimensional numerical model for main flow in a rocket nozzle. The model consists of solving continuity, momentum, and two energy equations for solid and fluid to obtain the fields of Darcy velocity, density, pressure, and temperatures of solid and fluid in the porous media. The numerical scheme used in the porous media solution is a finite difference method which couples the continuity and momentum equations to get the velocity-pressure field. Afterwards, it applies explicit and implicit methods to the transient form of the energy equation in solid phase. Having the solid temperature 
field calculated, it solves the transient energy equation of the fluid phase and finally, it updates the density field according to the equation of state. To validate the results of this model, the porous medium equations were considered and resolved in the Cartesian coordinates regarding the results obtained from the experimental study on transpiration cooling which was conducted in university of Stuttgart. On the other hand, assuming real conditions of a converging-diverging nozzle rocket nozzle, the main flow gas dynamic equations are solved along the nozzle considering a quasione-dimensional model. This model considers area variation, skin friction, heat transfer, and mass addition as the interaction with the porous media. It employs a high order numerical integration to obtain the Mach number distribution in the nozzle which leads the solution procedure to calculate the distribution of the other properties. At the interface of the main flow and the porous media (i.e. hot face), only convective heat transfer is assumed. The coefficient of convective heat transfer is obtained from some mathematical correlations in the literature [1]. The modeling is carried out by alternating the solution of hot-gas flow and porous medium flow while specifying the nozzle flow results as boundary conditions of the hot face of the porous layer.

\subsection{Thesis Organization}

Another part of this research, as an engineering analysis, concerns the study and analysis of the parameters affecting transpiration cooling process. These parametric studies make the results of the mathematical modeling valuable since they can examine a wide range of values for each parameter which can be infeasible or too expensive for experimental tests. For instance, parametric studies are developed to observe the heat flux distribution in the porous layer, examining the effect of coolant gas type, and sample thickness on transpiration cooling process. Also, numerical 
temperature results of thrust chamber wall and gas flow are used to calculate the coefficient of heat transfer through energy balance at the wall. 


\section{Chapter 2}

\section{LITERATURE REVIEW}

The beginning of studies on transpiration cooling for aerospace applications was in 1947. Rannie [2] developed an approach to analyze the temperature of the porous wall for laminar flow and compared his theory to some experimental results. The results were good only for porous substrates of low thermal conductivity due to lateral heat conduction in the samples. Eckert and Livingood [3] carried out studies and showed the superiority of transpiration cooling to the other methods such as film cooling. The Reynolds number range for turbulent flows was considered in their research as $\operatorname{Re}=10^{5}$ to $10^{9}$. Also, a Stanton number relationship was proposed for the hot side heat transfer. In their study, thermal equilibrium between the porous wall and the coolant was assumed and they neglected the radiation from the surroundings to the wall. Glass et al. [4] have conducted a numerical simulation of transpiration cooling with $\mathrm{C} / \mathrm{C}$ materials at scramjet combustor conditions using hydrogen as a coolant. Firstly, they calculated the heat flux reduction at the thrust chamber wall because of the blocking provided by the injection using a boundary layer code. The flow through the porous material is then simulated with the aid of a one-dimensional finite difference code for the temperatures of solid and coolant and the pressure of the coolant.

Meinert et al. [5] performed some researches on transpiration cooling with foreign gas injection. They specified the influence of foreign gas transpiration on skin friction and heat transfer. They created simple empirical correlations describing skin friction and heat transfer reduction depending on the blowing ratio, the temperature 
difference and especially the blowing gas properties. These studies were specifically focused on the heat transfer and friction of the main flow which can be affected by the transpired coolant injected to it. Numerical investigations of transpiration cooling of a cylindrical porous medium in a subsonic turbulent flow has been carried out by Mathelin [6]. In his research, a model of two-dimensional RANS applying Reynolds Stress Model of the hot gas flow is coupled with a one-dimensional porous media solver. They specified the flow rate and solved two temperature equations in the porous medium. Their results are compared with experimental results. As a conclusion thermal non-equilibrium between the coolant and the porous material is far from being negligible at least for lower blowing ratios. Tully [7] investigated transpiration cooling of an injector plate of liquid propellant rocket engines using a monolithic approach. Numerical simulations of both porous media and open channel flow are based on a semi heuristic model for the porous media flow which reduces to the Navier - Stokes equations for incompressible flow in the case of an open channel flow. In this research only air has been used as injected coolant and propellant.

More recent investigations took the porous media flow analysis and equations further into consideration. German Aerospace Center (DLR), for example, contributed to a variety of research projects about this cooling technique. Lezuo and Haidn [8] studied transpiration cooling experimentally and theoretically as a part of the Integrated High-Payoff Rocket Propulsion Technology Program (IHPRPT) at German Aerospace Center (DLR). They studied the performance of transpiration cooling under different operating conditions using a modular H2/O2-fired model combustor equipped with porous wall segments. Using $\mathrm{H}_{2}$ and $\mathrm{H}_{2} \mathrm{O}$ as the coolant, they also calculated Stanton numbers in order to obtain heat transfer coefficient 
based on their experiments. Beside of their experiments, they investigated numerical work to emphasize the development of the temperature transients inside the porous walls. Hannemann [9] presented a numerical approach for simulating the injection of different coolants into a laminar, hypersonic boundary layer. A simplified onedimensional model was used which assumed a given mass flux distribution and the temperature of the coolant being equal to the temperature of the surface of the porous wall. Results for different coolants were shown and the influence of the injection on the flow field was described.

Wang and Wang [10] studied analytical solution of transpiration cooling problems as a local thermal nonequilibrium (LTNE) one-dimensional model. They neglected the thermal conduction of the coolant within porous media. In their analytical solution process, two problems were investigated. At first, the effects of influential parameters of transpiration cooling were analyzed, which indicated that the cooling process were deeply affected by coolant mass flow rate, the Biot number at the hot surface of porous plate, and the Biot number in the pores. Second, the error caused by the assumption of the local thermal equilibrium (LTE) model was discussed. Based on the analytical solution and the error analysis, a quantitative criterion to choose the LTNE or LTE model was suggested, and the corresponding expression was also given in their paper.

Greuel and Herbertz [11] in German Aerospace Center, carried out an investigation on analyzing the performance of transpiration cooled thrust chamber for $\mathrm{C} / \mathrm{C}$ liners with hydrogen as the coolant. They proved the applicability of transpiration cooling to $\mathrm{C} / \mathrm{C}$ liners used in liquid rocket engines. They also studied the performance of a transpired cooled thrust chamber due to variation of coolant mass flow rate. Their 
numerical study was developing a two-dimensional CFD tool based on the commercial ANSYS software. They investigated simulation of compressible transpiration cooling besides experimental studies in order to obtain the coolant mass flow along a C/C liner. In a project by Cheuret [12], transpiration cooling has been investigated for Ramjet and Scramjet engines using a supersonic hot gas channel with a porous material integrated in the channel wall. In this research, a onedimensional numerical approach using transport equations for a turbulent flow adapted to a porous media flow was described. This procedure was monolithic with different coefficients in laminar and turbulent regimes. The results of this approach were compared with experimental results and found to be in a reasonable agreement concerning the cooling efficiency.

Sözen \& Davis [13], who have investigated transpiration cooling in a liquid rocket thrust chamber wall using numerical method, focused on incompressible flow model to which they applied implicit and explicit approximation methods in Cylindrical coordinates. They have also attempted to solve compressible flow model in a simple one-dimensional cylindrical domain, though they have not obtained acceptable results due to long time of running their computer code. Langener [14-16] also performed an experimental study of transpiration cooling using $\mathrm{C} / \mathrm{C}$ porous structure in Cartesian coordinates at the institute of Aerospace Thermodynamics at the University of Stuttgart. The major part of their research was embedded within the framework of the ATLLAS project and supported by experiments embedded within the SFB-TRR40 project. The C/C samples were first characterized with respect to their outflow and through-flow behavior in separate test setups. Then, these samples were exposed to heated supersonic and subsonic flows generating different heat 
loads. The surface temperature of the porous wall segments were determined using thermocouple measurements and calibrated infrared thermography. One of the significant parts of their research was studying one-dimensional temperature distribution of samples using different coolants such as Air and Helium. Gascoin et al. $[17,18]$ developed an approach to investigate the application of hydrocarbon fuel as a coolant fluid for transpiration cooling, including the effects of chemical reactions due to fuel pyrolysis. They used a one-dimensional numerical model and validated their model with experiments.

Dahmen et al. [19, 20] simulated transpiration cooling using ceramic matrix composite (CMC) materials for cooling rocket thrust chambers. Air as the coolant was injected through the porous material by a pressure difference between the coolant reservoir and thrust chamber. They demonstrated that the effectiveness of the cooling process depends on an appropriate choice of the parameters such as injection pressure, blowing ratios, and material structure parameters. They formulated a twodimensional model for the porous medium flow, and developed a finite element solver for the porous media flow. They coupled the finite element solver with a finite volume solver for the compressible Reynolds averaged turbulent Navier-Stokes equations.

Although most of the mentioned literature studied a porous liner in model rocket combustion chamber, more detailed information of the surface temperature distribution and temperature profile within the porous material are lacking. The mentioned researches consider not enough parameters which play key roles in the transpirations cooling process. Optimizing these parameters can significantly enhance the cooling performance and thus increasing the overall rocket engine 
efficiency. On the other hand, more realistic conditions of the main flow, regarding the rocket engine conditions, are necessary to be simulated and studied. Therefore, to approach the essence of this cooling method as a necessary sub-system of a rocket engine, one has to take the whole interactions of this process with the hot gas flow within the thrust chamber. 


\section{Chapter 3}

\section{POROUS MEDIA: SYSTEM DESCRIPTION \& EQUATIONS}

The major part of transpiration cooling process occurs as the interaction between coolant fluid and solid structure within the porous media. Thus, it would be beneficial to observe the thermal situation and heat exchange which can appear within a porous wall. To describe the thermal behavior of a porous wall, two energy equations would be necessary; however, in this research it is assumed that the fluid and solid structure are in thermal equilibrium within the porous medium. The coolant injection pressure must be more than that of in the thrust chamber at each section in order to move the fluid through the porous material towards the combustion chamber. In some cases, two layers are embedded inside the thrust chamber wall to increase the thermal protection: A liner which is placed adjacent to the hot gases, and then, a foam layer. The schematic illustration of transpiration cooled thrust chamber of a rocket in Fig.2 shows a hot gas flowing in chamber in contact with a permeable liner which is attached on the surface of the inner porous layer. 


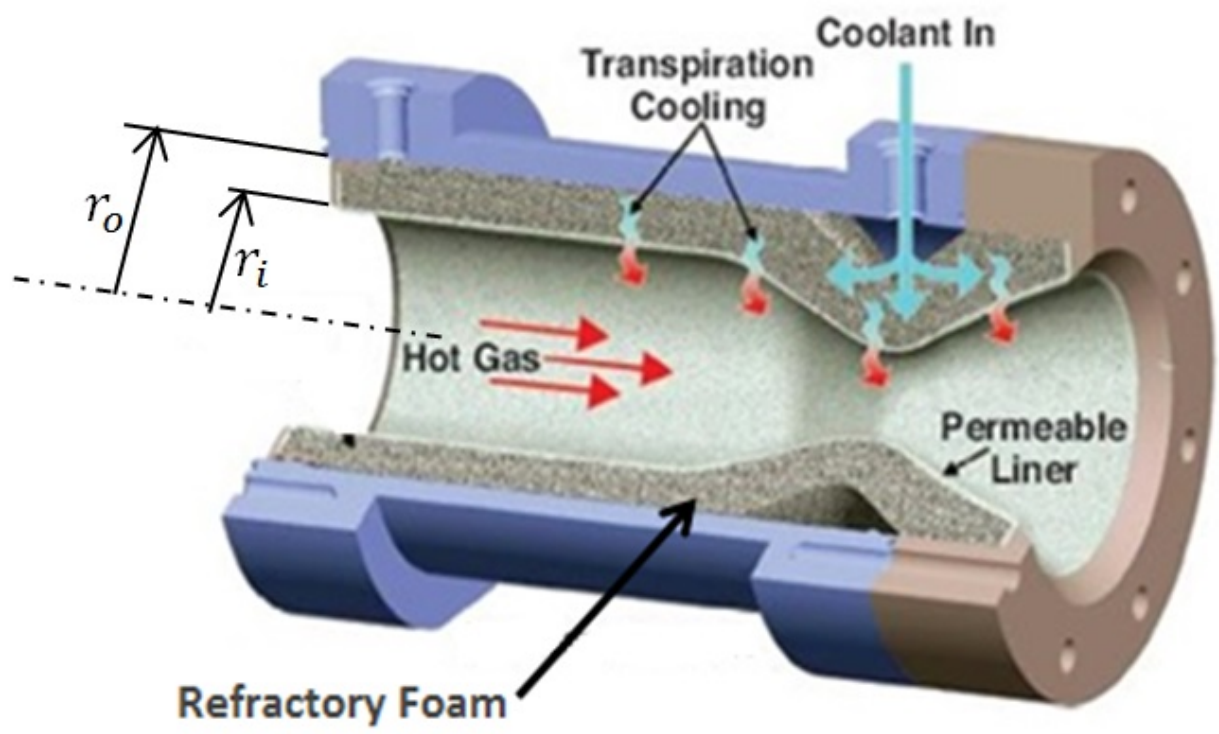

Figure 2: Schematic illustration of transpiration cooled thrust chamber of a rocket.

The second layer is refractory foam (i.e. porous layer) into which coolant is injected. As a matter of fact, the coolant flows within the foam towards the chamber in opposite direction of the heat flow (see Fig.2). This mechanism of cooling is fundamentally similar to a counter flow heat exchanger. The outer face, at which the coolant is injected, is called the cold face, and the inner face, which is adjacent the thrust chamber, is named the hot face. Unlike the pressure, the temperature is higher at the hot face because of high-temperature passing gases in the chamber and lower at the outer diameter. As the coolant flows through the porous sample, its velocity and temperature increase while its pressure reduces to the thrust chamber pressure. Figure.1 illustrates the problem geometry, especially the coordinates, direction of which the equations are to be solved, i.e. radial direction, and the boundary conditions. The coolant is injected to the domain from the outer (cold) boundary $\mathrm{r}_{\mathrm{o}}$ radially. The axial heat transfer and flow of the coolant are neglected. Also, convective boundary conditions at both coolant and hot-gas sides for the solid are shown. 


\subsection{Mathematical Modeling in Cylindrical System}

The equations describing the behaviors of the coolant and porous structure which involve conservation principles used in the analysis of transport phenomena in the porous media are presented. These equations are steady one-dimensional in cylindrical coordinates. In reality, the geometry is a hollow cylinder with the inner and outer radiuses of $r_{i}$ and $r_{0}$, respectively (see Fig.3).

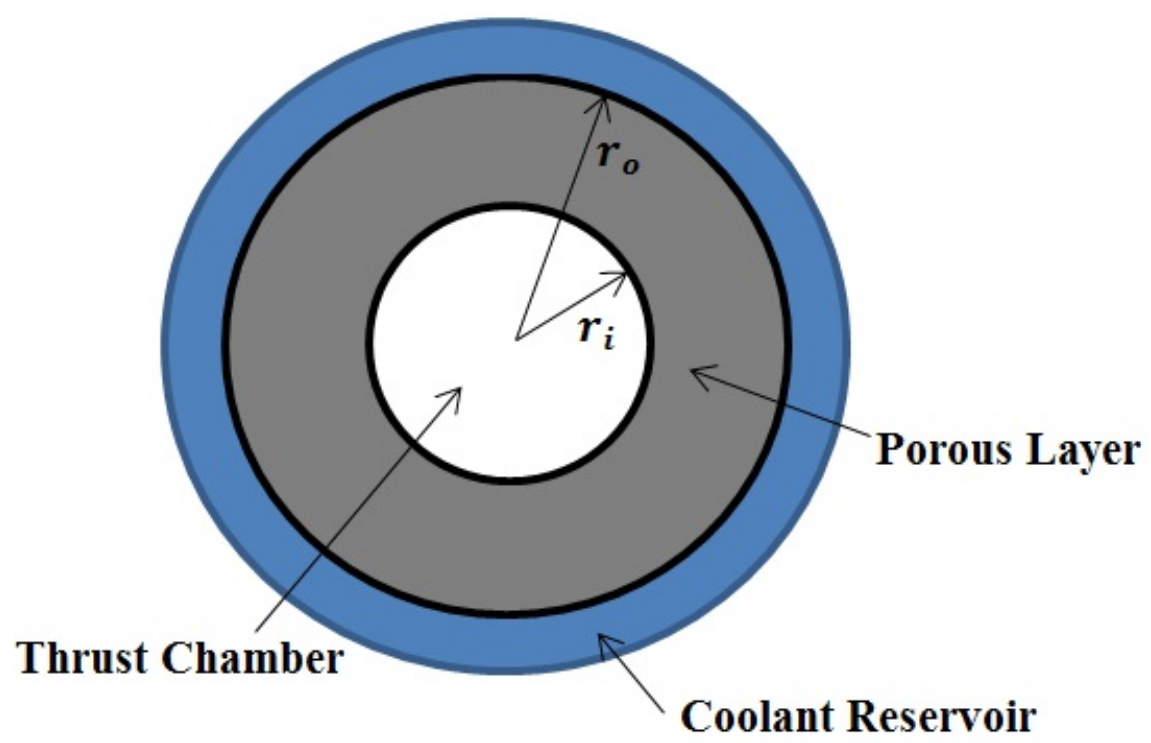

Figure 3: Schematic of porous layer and its inner/outer surfaces.

The governing equations are written in general form for compressible flow as follows.

Continuity Equation: The continuity equation can be written as the transient mass conservation equation in one-dimensional cylindrical domain

$\varepsilon_{A} \frac{\partial \rho_{f}}{\partial t}+\frac{1}{r} \frac{\partial}{\partial r}\left(r \rho_{f} u_{f}\right)=0$ 
In Eq. (1), $\varepsilon_{\mathrm{A}}$ is the average porosity and subscript $\mathrm{f}$ denotes fluid properties, respectively.

Momentum Equation: The conservation of momentum, which is also known as Darcy-Forchheimer equation, for a one-dimensional cylindrical domain through a porous material can be expressed as

$\frac{1}{\varepsilon_{A}} \frac{\partial}{\partial t}\left(\rho_{f} u_{f}\right)+\frac{\partial P_{f}}{\partial r}=-\left(\frac{\mu_{f}}{\alpha_{A}}+\frac{\rho_{f}}{\beta_{A}}\left|u_{f}\right|\right) u_{f}$

where, $\alpha_{A}$ and $\beta_{A}$ are the permeability coefficients and can be approximated by the following correlations [21]

$\alpha_{A}=\frac{D_{p}^{2} \varepsilon_{A}^{3}}{150\left(1-\varepsilon_{A}\right)^{2}}$

$\beta_{A}=\frac{D_{p} \varepsilon_{A}^{3}}{1.75\left(1-\varepsilon_{A}\right)}$

Normally, regarding the solution method of Navier-Stokes equations, due to the format of Eqs.1 and 2, they can be solved to yield the density and velocity fields, respectively. However, according to the previous studies and because of the specific conditions of transpiration cooling such as low range of mass flow rate and the porous sample thickness, the flow is strictly laminar. The low range of Reynolds number within the porous medium calculated in the later sections proves this fact. Therefore, due to density variation within the sample, it is a "weak compressible flow” and solving continuity and momentum equations to get density and velocity will not be helpful [22]. An appropriate method of solving this kind of flow is to solve the steady form of continuity equation for Darcian velocity, and afterwards, solving the steady form of momentum equation for pressure, and obtain density from 
the equation of state [22]. As a result, it would be more appropriate if Eqs.1 and 2, are stated in the steady form as

$\frac{\partial}{\partial r}\left(r \rho_{f} u_{f}\right)=0$

$\frac{\partial P_{f}}{\partial r}=-\left(\frac{\mu_{f}}{\alpha_{A}}+\frac{\rho_{f}}{\beta_{A}}\left|u_{f}\right|\right) u_{f}$

Energy Equation: It is assumed that, despite of minor difference between their temperatures, solid and fluid within the porous media are in thermal equilibrium (see Fig.3). Therefore, only one energy equation has to be solved for both the solid and the fluid parts [21].

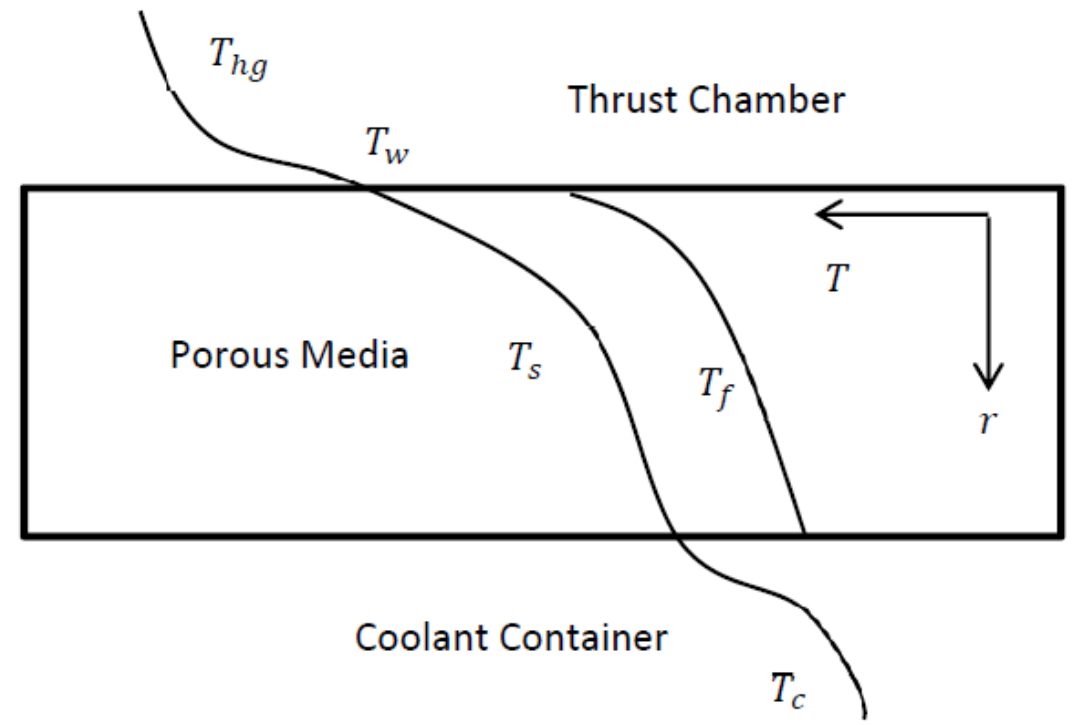

Figure 4: Temperature profile within the domain.

This energy equation for one-dimensional cylindrical coordinates assuming thermal equilibrium between the two phases can be expressed as

$\left(\rho c_{p}\right)_{A} \frac{\partial T}{\partial t}+\varepsilon_{A} \rho_{f} u_{f} c_{p f} \frac{\partial T}{\partial r}=\frac{1}{r} \frac{\partial}{\partial r}\left(k_{A} r \frac{\partial T}{\partial r}\right)$

and the steady form of Eq. (7) is demonstrated as 
$\varepsilon_{A} \rho_{f} u_{f} c_{p f} \frac{\partial T}{\partial r}=\frac{1}{r} \frac{\partial}{\partial r}\left(k_{A} r \frac{\partial T}{\partial r}\right)$

where, the parameters $k_{A}$ and $\left(\rho c_{p}\right)_{A}$ are expressed as

$$
\begin{aligned}
& k_{A}=\frac{k_{f}\left[\left(2 k_{f}+k_{S}\right)-2\left(1-\varepsilon_{A}\right)\left(k_{f}-k_{s}\right)\right]}{2 k_{f}+k_{S}+\left(1-\varepsilon_{A}\right)\left(k_{f}-k_{S}\right)} \\
& \left(\rho c_{p}\right)_{A}=\left(1-\varepsilon_{A}\right) \rho_{S} C p_{s}+\varepsilon_{A} \rho_{f} C p_{f}
\end{aligned}
$$

The parameter $k_{f}$ and $c p_{f}$ are assumed to be functions of temperature, these functions are obtained through thermos-physical tables of Hydrogen (the assumed coolant in this research) as [23, 24]

$$
\begin{aligned}
& C p_{f}(T)=\frac{1000}{2.016} \cdot\left(29.11-\left(0.1916 \times 10^{-2}\right) T+\left(0.4003 \times 10^{-5}\right) T^{2}-\right. \\
& \left.\left(0.8704 \times 10^{-9}\right) T^{3}\right) \\
& k_{f}(T)=419 \times 10^{-5} \cdot\left(20.37-\left(8.2 \times 10^{-2}\right) T+\left(3.56 \times 10^{-6}\right) T^{2}\right)
\end{aligned}
$$

In Eq. (8), the LHS includes the convective term of fluid and the RHS contains the conductive term.

Equation of State: The fluid density is a function of its pressure and temperature. Treating the coolant fluid as a perfect gas, the equation of state can be applied to determine the density regarding the pressure and temperature.

$$
P_{f}=\rho_{f} R T_{f} \rightarrow \rho_{f}=\frac{P_{f}}{R T_{f}}
$$

\subsection{Numerical Method}

As mentioned earlier, the conservation equations of mass, momentum, and energy create a weak compressible flow modeling in the porous sample and. According to 
the nature of these equations concerning parabolic form of fluid energy equation, also, low range of Darcian velocity within the porous media, the flow does not contain any discontinuities. A second order finite difference approximation (i.e. Eq. (14)) is applied to Eqs.5 and 6 so that the velocity and pressure fields are achieved, respectively.

$\frac{\partial \varphi_{i}^{n}}{\partial r}=\frac{-3 \varphi_{i}^{n}+4 \varphi_{i+1}^{n}-\varphi_{i+2}^{n}}{2 \Delta r} \quad i=1,2, \ldots, N-2$

Equation (14) can only be run for the specified nodes (i.e. $i=1,2, \ldots, N-1$ ), since it is a second order forward difference approximation. To take the node $\mathrm{N}-1$ into consideration, a linear interpolation between node $\mathrm{N}-2$ and cold boundary (i.e. node $\mathrm{N}$ ) is made (see Fig.4).

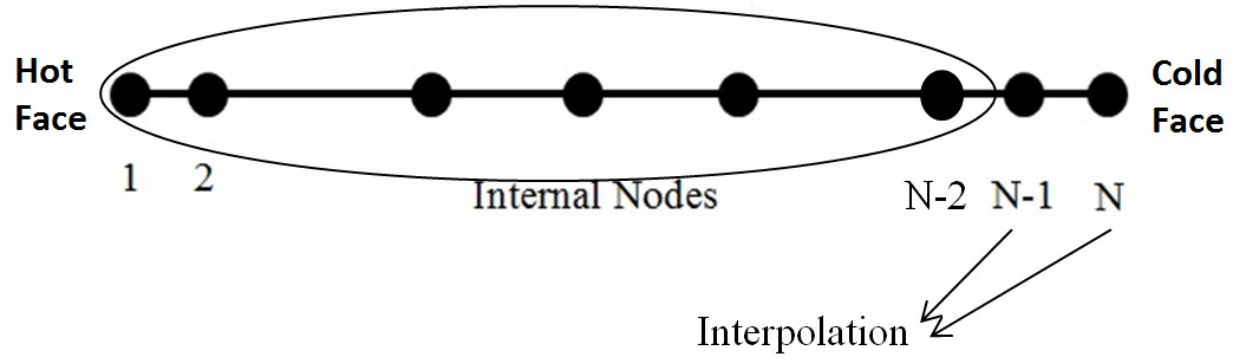

Figure 5: Grid points within the porous medium, internal and interpolated nodes.

Subsequently, a second order central difference approximations (i.e. Eq. (15)) is used to discretize the energy equation (i.e. Eq. (7)) for both convective and conductive terms [25]. This approximation can be performed assuming two nodes $\mathrm{i}-1 / 2$ and $\mathrm{i}+1 / 2$ and applying to the first and second order derivative terms of Eq. (7). As to the first order derivative term (i.e. convective term), Eq. (14) can be expressed as 
$\frac{\partial T}{\partial r}=\frac{T_{i+1 / 2}-T_{i-1 / 2}}{\Delta r} \quad i=2, \ldots, N-1$

and for second order derivatives (i.e. conductive term) is expressed as

$\frac{\partial}{\partial r}\left(k_{A} r \frac{\partial T}{\partial r}\right)=\frac{\left(k_{A} r \frac{\partial T}{\partial r}\right)_{i+1 / 2}-\left(k_{A} r \frac{\partial T}{\partial r}\right)_{i-1 / 2}}{\Delta r} \quad i=2, \ldots, N-1$

This approximation method imports the conductivity change with respect to the temperature at each node as well as the other terms. Equation (16) could be then simplified to

$\frac{\left(k_{A} r \frac{\partial T}{\partial r}\right)_{i+1 / 2}-\left(k_{A} r \frac{\partial T}{\partial r}\right)_{i-1 / 2}}{\Delta r}=\frac{\left[k_{A i+1 / 2} \cdot r_{i+1 / 2} \cdot \frac{T_{i+1}-T_{i}}{\Delta r}\right]-\left[k_{A i-1 / 2} \cdot r_{i-1 / 2} \cdot \frac{T_{i}-T_{i-1}}{\Delta r}\right]}{\Delta r}$

Therefore, Eq. (7) can be discretized as

$\varepsilon_{A} r c_{p f} \rho_{f} u_{f} \frac{T_{i+1}-T_{i-1}}{2 \Delta r}=\frac{\left[k_{A i+1 / 2} \cdot r_{i+1 / 2} \cdot \frac{T_{i+1}-T_{i}}{\Delta r}\right]-\left[k_{A i+1 / 2} \cdot r_{i-1 / 2} \cdot \frac{T_{i}-T_{i-1}}{\Delta r}\right]}{\Delta r}$

Finally, the approximations lead the energy equation to the following tri-diagonal form as

$$
\begin{aligned}
& -\left(\varepsilon_{A} r c_{p f} \rho_{f} u_{f}+\frac{2 k_{A i-1 / 2} \cdot r_{i-1 / 2}}{\Delta r}\right) T_{i-1}+\left(\frac{2 k_{A_{i+\frac{1}{2}}} \cdot r_{i+\frac{1}{2}}}{\Delta r}+\frac{2 k_{A i-1 / 2} \cdot r_{i-1 / 2}}{\Delta r}\right) T_{i}+\left(\varepsilon_{A} r c_{p f} \rho_{f} u_{f}-\right. \\
& \left.\frac{2 k_{A i+1 / 2} \cdot r_{i+1 / 2}}{\Delta r}\right) T_{i+1}=0
\end{aligned}
$$

Equation (19) is to be solved for the internal nodes of $i=2,3, \ldots, N-1$.

\subsection{Initial and Boundary Conditions}

The sample is assumed to be a hollow cylinder where the coolant is flowing radially through. As shown in Fig.2, the coolant is maintained at a specified pressure $\mathrm{P}_{\mathrm{c}}$ and 
temperature of $T_{c}$ and injected at the outer surface (i.e. cold face) of the cylinder. Therefore, the pressure and temperature at the cold face can be directly obtained from these conditions, while, the velocity comes from continuity equation. Density at the cold face is, then, calculated from the equation of state. The boundary conditions at the cold face can be presented as

$$
\begin{aligned}
& P\left(r_{o}\right)=P_{c} \\
& T\left(r_{o}\right)=T_{c} \\
& \rho_{f}\left(r_{o}\right)=\frac{P\left(r_{o}\right)}{R T\left(r_{o}\right)} \\
& u_{f}\left(r_{o}\right)=\frac{\dot{m}_{c}}{\rho\left(r_{o}\right) \cdot\left(A_{c}\right)}
\end{aligned}
$$

In the equations above, $P_{c}$ and $T_{c}$ are specified pressure and temperature of the coolant at the injection surface (cold face), respectively. Also, $A_{c}=2 \pi r_{o}$ is the side area of the porous hollow cylinder at the outer (cold) face, where the coolant is injected.

On the other hand, at the inner surface (i.e. hot face) the pressure can be obtained from solving equation of Mach number (i.e. Eq. (39)) within the thrust chamber. Solution of momentum equation in the thrust chamber is presented in the next section.

$P\left(r_{i}\right)=P_{h g}$

$\mathrm{P}_{\mathrm{hg}}$ is the local gas flow pressure in the thrust chamber which is generally obtained from Eq. (39). Temperature can also be found from the energy balance at the interface as 
$-k_{A} \frac{d T}{d r}=h_{g}\left(T\left(r_{i}\right)-T_{h g}\right)$

In Eq. (25), $T_{\text {hg }}$ is the local gas temperature in thrust chamber which is also obtained from Eq. (39) in thrust chamber. Parameter $h_{g}$ is the coefficient of heat transfer at the hot face which is obtained from Eq. (29) proposed by Kays [1]. Kays derived a correlation for Stanton number at the thrust chamber wall which is exposed to transpired cooling flow. The relation is expressed as

$\frac{S t_{h g}}{S t_{h g, 0}}=\frac{b_{h}}{e^{b_{h-1}}}$

$b_{h}=\frac{F}{S t_{h g, 0}}$

where, $\mathrm{St}_{\mathrm{hg}, 0}$ denotes the Stanton number for the case of no transpiration cooling and F represents blowing ratio $\left(F=\frac{(\rho u)_{\text {coolant }}}{(\rho u)_{\text {main flow }}}\right)$. It can be estimated through a correlation presented by Dittus, for the case of heat transfer of fully developed turbulent flow in ducts as follows [26]

$S t_{h g, 0}=0.026 R e_{h g}^{-0.2} P r_{h g}^{-0.6}$

when the Stanton number is calculated and the heat transfer coefficient can be obtained from

$h_{g}=S t_{h g} \cdot \rho_{h g} \cdot c_{p, h g} \cdot V_{g}$

Finally, density and Darcian velocity can be calculated as

$$
\begin{aligned}
& \rho_{f}\left(r_{i}\right)=\frac{P\left(r_{i}\right)}{R T\left(r_{i}\right)} \\
& u_{f}\left(r_{i}\right)=\frac{\dot{m}_{c}}{\rho\left(r_{i}\right) \cdot\left(A_{\text {exit }}\right)}
\end{aligned}
$$


In Eq. (31), $A_{\text {exit }}=2 \pi r_{i}$ denotes the side area of the porous hollow cylinder at the inner face.

\subsection{Mathematical Modeling in Cartesian System and Benchmarking}

The experimental results, as pointed out earlier, are obtained from an experimental study on transpiration cooling in Cartesian coordinates for C/C liners, which were prepared as rectangular plates, by Langener $[15,16]$. Therefore, prior to solving the equations in cylindrical coordinates, they are solved in Cartesian coordinates for comparing their results with the experimental results. The goal of their study was to test and qualify $\mathrm{C} / \mathrm{C}$ ceramic matric materials (i.e. CMC) for the application in rocket or scramjet engines. A part of their study was to obtain the temperature profiles and cooling efficiencies for different coolant mass flow rates for the case of subsonic main flow. Also, they assumed thermal equilibrium condition between solid and fluid within the porous medium. It means that the internal convective heat transfer coefficient is at its maximum level maintaining the solid and fluid temperatures almost equal. In this case the energy equation within the porous medium can be expressed as

$\varepsilon_{A} \rho_{f} u_{f} c_{p f} \frac{\partial T}{\partial x}=\frac{\partial}{\partial x}\left(k_{A} \frac{\partial T}{\partial x}\right)$

The term $\mathrm{k}_{\mathrm{A}}$ denotes the effective thermal conductivity of the solid and fluid phases in local thermal equilibrium. In this research, an approximation was used according to the model proposed by Chi [27] as

$k_{A}=\frac{k_{f}\left[\left(2 k_{f}+k_{S}\right)-2\left(1-\varepsilon_{A}\right)\left(k_{f}-k_{S}\right)\right]}{2 k_{f}+k_{S}+\left(1-\varepsilon_{A}\right)\left(k_{f}-k_{S}\right)}$

\subsection{Experimental Study}


According to the experimental study $[15,16]$, the inner face is exposed to convective heat flux caused by the main hot flow and the hot face temperature is $T_{\mathrm{w}}$. Also, the coolant flow which is maintained at $T_{c}$, is injected from the outer face called cold face (see Fig.5).

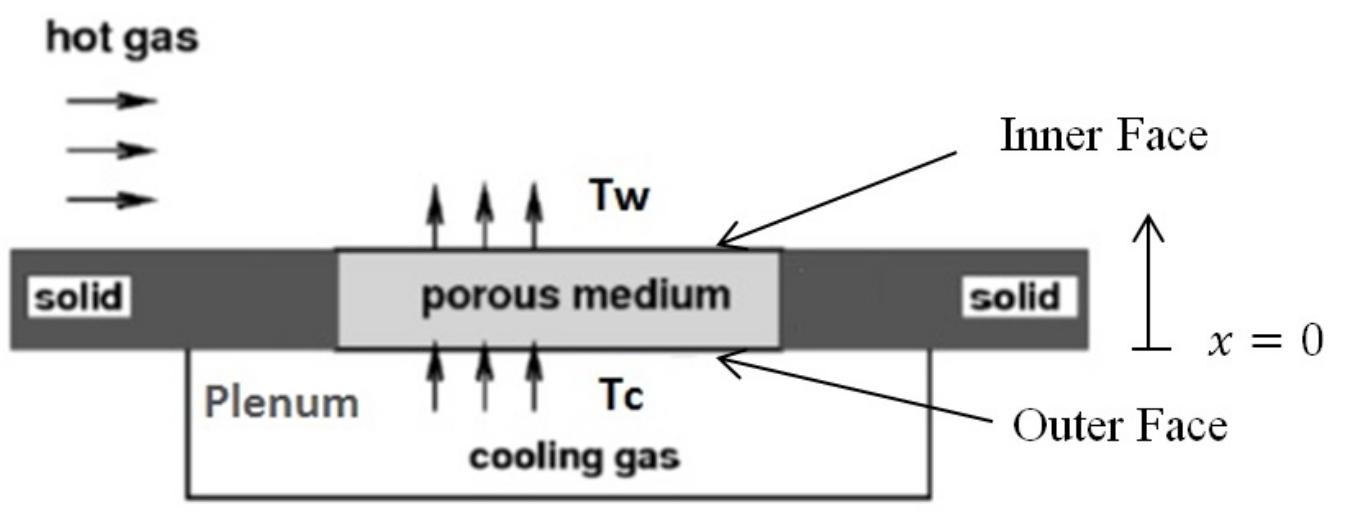

Figure 6: Experimental Setup.

They measured the hot face temperature at the wall (i.e. hot wall temperature) at different points and took the average of them as the constant wall temperature. Thus, their hot face boundary condition was a constant temperature $\mathrm{T}_{\mathrm{w}}$. Therefore, the boundary conditions are then as

$$
\begin{aligned}
& x=0 \rightarrow T=T_{c} \\
& x=\mathrm{L} \rightarrow T=T_{w}
\end{aligned}
$$

Regarding their case of subsonic flow and Mach number equal to 0.5, they estimated a value of 0.0030 for $\mathrm{St}_{\mathrm{g}, 0}$ which is corresponding to the convection heat flux of $q_{0}=21083 \mathrm{~W} / \mathrm{m}^{2}$ (i.e. no transpiration cooling). They also defined a blowing ratio $\mathrm{F}$, which is the ratio of mass flux of the coolant to that of the hot gases. A rectangular C/C sample, corresponding to the sample PH1606-1 and air was assumed to be the 
porous structure and the coolant respectively. The properties of the sample are demonstrated in the Table 1.

Table 1: Investigated Sample PH1606-1 [15].

\section{C/C Porous}

Property

\section{Material}

\begin{tabular}{lc}
\hline $\mathbf{L}[\mathbf{m}]$ & 0.015 \\
$\boldsymbol{\varepsilon}[\%]$ & 11.3
\end{tabular}

\begin{tabular}{cc}
$\mathbf{k}_{\mathbf{s}}[\mathbf{W} / \mathbf{m} . \mathbf{K}]$ & 1.4 \\
$\mathbf{K}_{\mathbf{D}}\left[\mathbf{m}^{2}\right]$ & $1.25 \cdot 10^{-13}$ \\
$\mathbf{K}_{\mathbf{F}}[\mathbf{m}]$ & $0.88 .10^{-8}$ \\
\hline
\end{tabular}

Five different blowing ratios of air, as the coolant, were applied to the sample. According to each mass flow rate, the cold and hot boundary temperatures were measured. Table 2 shows these mass flow rates (blowing ratios and mass flow rates) and the corresponding temperatures.

Table 2: Different mass flow rates and corresponding boundary temperatures [15].

\begin{tabular}{cccc}
\hline $\mathbf{F}$ & $\dot{\boldsymbol{m}}(\mathrm{gr} / \mathrm{s})$ & $\mathbf{T}_{\text {hot }}(\mathbf{K})$ & $\mathbf{T}_{\text {cold }}(\mathbf{K})$ \\
\hline 0 & 0.0 & 475.5 & 368.3 \\
0.001 & 0.555 & 453.5 & 336.3 \\
0.002 & 1.091 & 423.9 & 319.1 \\
0.003 & 1.616 & 399.2 & 315.5 \\
0.01 & 5.332 & 324.0 & 296.6 \\
\hline
\end{tabular}

\subsection{Comparison of Experimental and Numerical Results}


The temperature profiles of both numerical and experimental test versus the nondimensional sample length in Fig.6 demonstrates that the numerical results are in a very good agreement with that of experimental results.

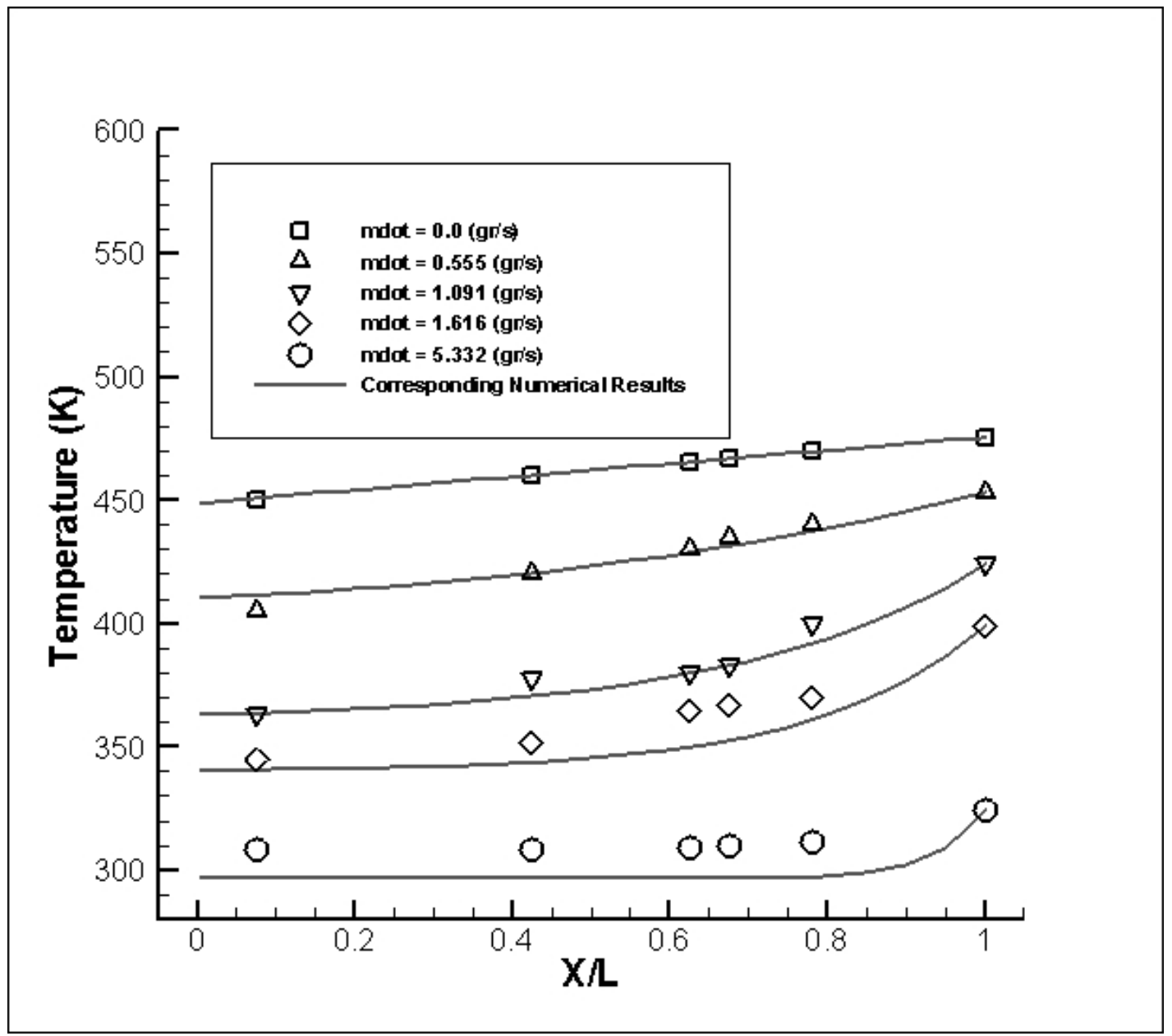

Figure 7: Temperature profile of the numerical solution of compressible flow in the Cartesian system (solid lines) compared with the experimental results (symbols) considering different Air mass flow rates.

Assuming that the properties are invariant of temperature, this is a linear second order ordinary differential equation which results the temperature as an exponential function of $x$. In Fig.6, the solid lines and the symbols represent the numerical solutions and experimental results respectively. For the especial case of $\left(\dot{m}_{c}=0\right)$ in which there is no coolant flow, the convective term in the energy equation is 
vanished. Under such a condition, conduction is the only mechanism of heat transfer and the temperature profile is nearly linear which is modeled by the numerical results very well. As the mass flow rate of the coolant increases, the effect of transpiration cooling becomes more obvious. Regarding the last mass flow rate of the coolant, the temperature is obtained almost at the temperature of the cold face, except for the points near the hot face. 


\section{Chapter 4}

\section{THRUST CHAMBER: SYSTEM DESCRIPTION \& EQUATIONS}

As the main part of the propulsion system of a rocket engine, gas products are flowing and accelerating within the nozzle in order to provide its thrust force. In this section, a converging-diverging part of a sample thrust chamber is considered (see Fig.7). To obtain the velocity (Mach number), pressure, and temperature fields within the nozzle, one has to solve the equations of continuity, momentum, and energy. Different methods can be used to solve these equations, for instance, the simplest way is to assume the whole engine space as a control volume and apply the corresponding continuity and momentum equations. This method yields the average pressure and thrust of the rocket engine, but, does not provide the detailed properties of the hot-gas flow. 


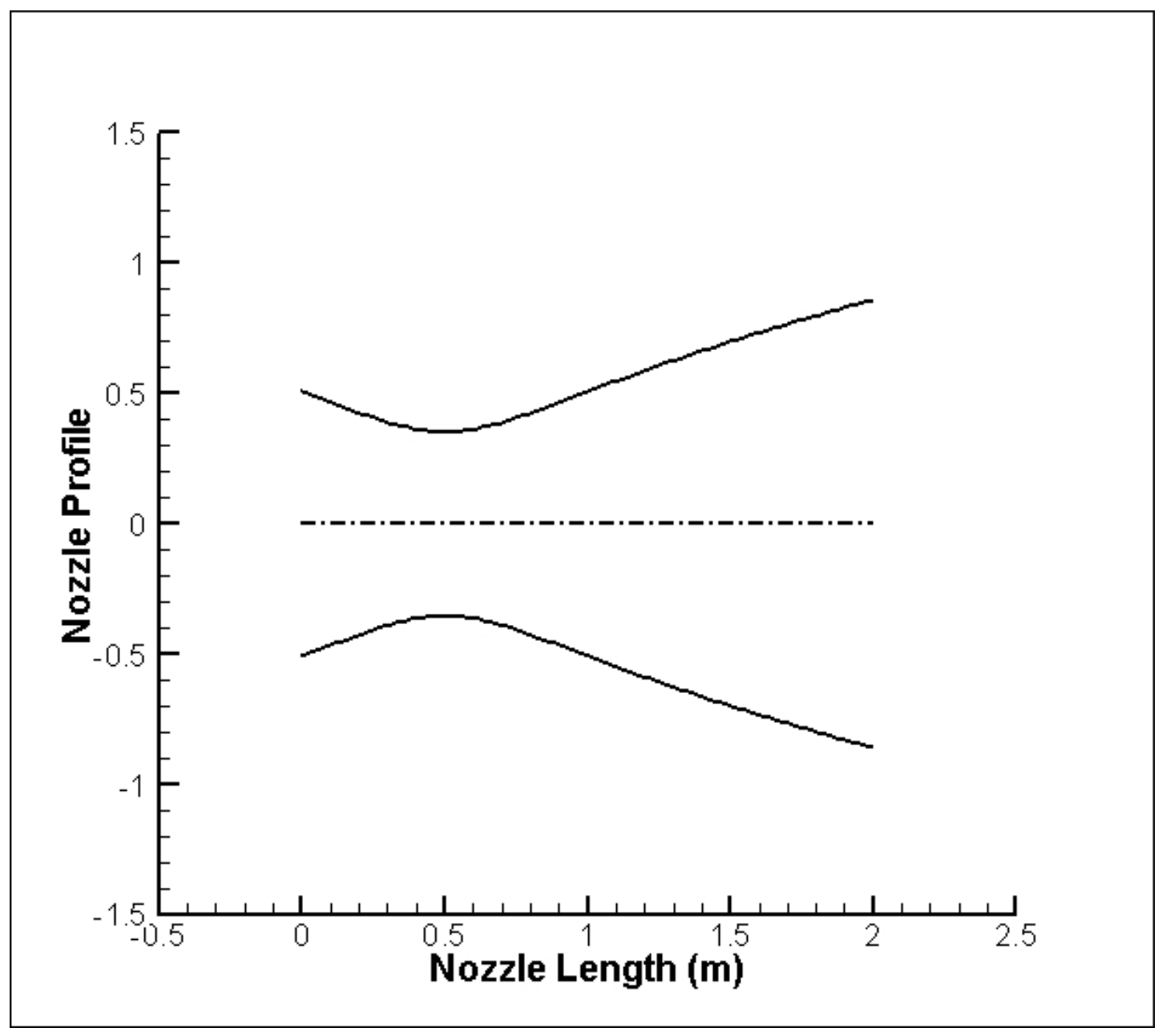

Figure 8: Profile of the converging-diverging nozzle.

\subsection{Mathematical modeling}

As the transpiration cooling occurs, the coolant enters the nozzle while absorbing the incoming heat flux to the walls. According to the interaction between the nozzle and the porous media, it is assumed that there is mass addition, friction, and heat transfer to the nozzle (work addition to the control volume is neglected). An infinitesimal control volume of the flow within the thrust chamber is considered and shown in Fig.8. The effects of heat and mass addition, friction, and area change are indicated in this figure [28]. 


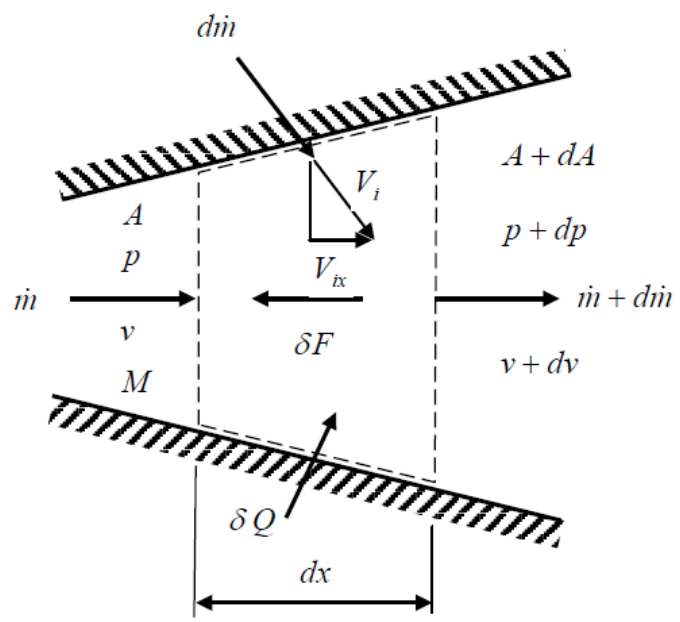

Figure 9: An infinitesimal control volume within the nozzle.

Applying mass, momentum, and energy conservation equations, the following differential equation can be obtained. It yields Mach number differential change as a function of area variation, friction factor, stagnation temperature and mass flow rate differential changes [28].

$\frac{d M}{M}=\frac{\psi}{1-M^{2}}\left[-\frac{d A}{A}+\left(\frac{\gamma M^{2}}{2}\right)\left(\frac{4 f d x}{D_{h}}\right)+\frac{\left(1+\gamma M^{2}\right)}{2} \frac{d T_{t}}{T_{t}}+\left(1+\gamma M^{2}\right) \frac{d \dot{m}}{\dot{m}}\right]$

In the equation above, parameter $\gamma$ is the gas constant and $\psi$ can be obtained from $\psi=1+\frac{\gamma-1}{2} \cdot M^{2}$

Also, $T_{t}$ represents stagnation temperature and the term $\frac{d T_{t}}{T_{t}}$ can be calculated from [28]

$$
\begin{aligned}
& \frac{d T_{t}}{T_{t}}=\frac{1}{\psi} \cdot\left(\frac{\delta Q-d H_{i}}{H}\right) \\
& d H_{i}=\left(H-H_{i}\right) \frac{d \dot{m}}{\dot{m}}
\end{aligned}
$$


In Eq. (37), $\delta Q$ is the differential heat transfer to the control volume, $H$ is enthalpy of the main flow which is $H=C_{p} T$. Also, $d H_{i}$ is the net specific enthalpy entering the control volume and $\mathrm{T}$ is the local gas temperature. In order to have a mathematical analysis of the heat transfer to the porous wall (as the boundary condition), a onedimensional solution method is chosen which yields reliable results. From a onedimensional viewpoint, the nozzle can be divided to some control volume elements to apply Eq. (36). Figure.7 illustrates the schematic of a converging-diverging nozzle in which the flow properties are assumed to vary in axial direction. The profile of the nozzle is expressed in the section 3.3. In other words, property variation in lateral direction is neglected. Therefore, the distribution of the properties is stored in the cell center of each cell. Rearranging Eq. (36) with respect to the nozzle length, gives the following form as

$\frac{d M}{d x}=\frac{\psi M}{1-M^{2}}\left[-\frac{1}{A} \frac{d A}{d x}+\left(\frac{\gamma M^{2}}{2}\right)\left(\frac{4 f}{D_{h}}\right)+\frac{\left(1+\gamma M^{2}\right)}{2 T_{t}} \frac{d T_{t}}{d x}+\left(1+\gamma M^{2}\right) \frac{1}{\dot{m}} \frac{d \dot{m}}{d x}\right]=f(M, x)$

Equation (39) needs to be integrated along x which yields the distribution of Mach number in the nozzle. Recalling that, Eq. (39), which is a first order ordinary differential equation with respect to the length of the nozzle, is an initial value problem (IVP). Also, for calculating the stagnation temperature distribution along the nozzle, Eq. (37) should be integrated. Having Mach number and stagnation profile determined in the nozzle, one may calculate the temperature distribution using the following temperature ratio as a function of Mach number and stagnation temperature [28].

$$
\frac{T_{2}}{T_{1}}=\frac{T_{t 2}\left(1+\frac{\gamma-1}{2} M_{1}^{2}\right)}{T_{t 1}\left(1+\frac{\gamma-1}{2} M_{2}^{2}\right)}
$$


Equation (40), represents the relation between temperatures of two adjacent grid points in nozzle (i.e. subscriptions 1 and 2) according to their Mach numbers.

\subsection{Numerical Method}

Eq. (39) is a general equation including all the terms of area change, skin friction, stagnation temperature change, and mass addition to the nozzle in ' $\mathrm{x}$ ' direction. Therefore, to integrate this equation, the mentioned terms have to either be expressed as a function of ' $x$ ' or difference form. Since the upstream flow condition in the nozzle is known, a backward difference approximation can be used to calculate the derivative terms. For instance, the term $\frac{d A}{d x}$ and $\frac{d \dot{m}}{d x}$ can be calculated as

$$
\begin{aligned}
& \frac{d \dot{m}}{d x}=\frac{\dot{m}_{i}-\dot{m}_{i-1}}{\Delta x} \\
& \frac{d A}{d x}=\frac{A_{i}-A_{i-1}}{\Delta x}
\end{aligned}
$$

Uniform grid division is then applied to the nozzle which points are used to store the properties of each. Assuming that the first grid point is the upstream point at which all the properties such as Mach number, temperature, and pressure are given. Then a $4^{\text {th }}$ order Runge-Kutta method is used to integrate Eq. (39) along the nozzle. To implement the RK4 method on this IVP (i.e. Initial Value Problem), initial conditions are needed in order to start the solution. By integrating this equation, Mach number of each point can be determined from its previous Mach number. Finally, the Mach number in the entire entire nozzle is calculated. During this procedure, the stagnation temperature and temperature of each point can be found from Eqs.37 and 40.

\subsection{Transonic Region}

Reconsidering Eq. (39) reveals that in the case of Mach number approaching unity, the value of $d M / d x$ rises infinitely and creates difficulties in the solution process. 
Thus, using a specific technique is necessary to pass the transonic region successfully without experiencing any discontinuities in the solution. An effective method, explained in the literature is using the L'Hopital's rule [28]. In this method, the derivative term of Mach number can be expressed as [28]

$\frac{d M}{d x}=\frac{\Lambda(x)}{1-M^{2}}$

where the term $\Lambda(\mathrm{x})$ is as

$\Lambda(x)=\psi M\left[-\frac{1}{A} \frac{d A}{d x}+\left(\frac{\gamma M^{2}}{2}\right)\left(\frac{4 f}{D_{h}}\right)+\frac{\left(1+\gamma M^{2}\right)}{2 T_{t}} \frac{d T_{t}}{d x}+\left(1+\gamma M^{2}\right) \frac{1}{\dot{m}} \frac{d \dot{m}}{d x}\right]$

Then, the L'Hopital rule can be applied toEq. (43) as

$\left(\frac{d M}{d x}\right)^{*}=\lim _{M \rightarrow 1}\left(\frac{d M}{d x}\right)=\frac{\frac{d}{d x}(\Lambda(x))^{*}}{-2\left(\frac{d M}{d x}\right)^{*}}$

In the above equation, the terms with the superscript of star $\left(^{*}\right)$, are the parameters calculated in the transonic region. Eq. (45) can be rearranged as

$\left[\left(\frac{d M}{d x}\right)^{*}\right]^{2}+\frac{1}{2}\left(\frac{d \Lambda}{d x}\right)^{*}=0$

The term $\frac{d \Lambda}{d x}$, includes the first derivative of Mach number and thus, Eq. (46) forms a quadratic equation of

$\left[\left(\frac{d M}{d x}\right)^{*}\right]^{2}+B\left(\frac{d \mathrm{M}}{d x}\right)^{*}+C=0$

where, the coefficients $\mathrm{B}$ and $\mathrm{C}$ are obtained from the derivative term of $\frac{d \Lambda}{d x}$. To choose the appropriate solution of the above equation, the downstream flow conditions have to be concerned.

\subsection{Verification}


In order to validate the results of this method, different cases are selected to be solved accordingly. A sample converging-diverging nozzle, having circular cross section and length of $2 \mathrm{~m}$, is chosen with the profile of

$y(x)=0.07-\left(\frac{0.1+(x-0.5)^{2}}{\pi}\right)^{0.25}$

As mentioned earlier, the nozzle profile is illustrated in Fig.8, and the throat is located at $\mathrm{x}=0.5 \mathrm{~m}$. Three cases are defined and studied for result verification.

First, the simple case of isentropic flow within the nozzle is considered. In this case, the variation of the Mach number is only because of area variation. This means that all parameters related to friction, stagnation temperature change, and mass additions are ignored. Thus, Eq. (39) can be rearranged as

$\frac{d M}{d x}=\frac{\psi M}{1-M^{2}}\left[-\frac{1}{A} \frac{d A}{d x}\right]=f(M, x)$

Also, cross sectional area of the nozzle can be calculated as $\mathrm{A}=\pi \mathrm{y}^{2}$. As explained before, Eq. (49) has to be integrated with a RK4 method. To do this, the initial conditions have to be determined.

Regarding the nozzle profile, the ratio of inlet area to the area of throat (i.e. $A_{i} / A^{*}$ ) can be determined as

$\frac{A_{i}}{A^{*}}=\frac{\pi y_{i}^{2}}{\pi y_{\text {throat }}^{2}}=2.054$

Water vapor $(\gamma=1.33)$ is assumed to be flowing within the nozzle, and then, from isentropic tables the inlet Mach number and the other ratios are obtained as

$M_{1}=0.301, \frac{T_{1}}{T_{t}}=0.98668, \quad \frac{P_{1}}{P_{t}}=0.94355$ 
The other initial conditions of the nozzle at the inlet (i.e. nozzle inlet conditions) as

$T_{1}=986.68 \mathrm{~K}, P_{1}=9.44 \times 10^{5} \mathrm{~Pa}$

Using a uniform grid size of 121 nodes, the integration of Eq. (49) can be performed to obtain the Mach number distribution. Subsequently, applying Eq. (40) besides the isentropic relation between pressure and temperature, the temperature and pressure profiles can also be calculated. Imposing the above initial conditions establishes design conditions of the nozzle in which the Mach number is unity at the throat. The results of Mach number, temperature and pressure profiles are obtained and, then, converted to non-dimensional form regarding their values at the throat. Fig.9 presents these results and shows the variation of the Mach number, temperature, and pressure with respect to the non-dimensional nozzle length. 


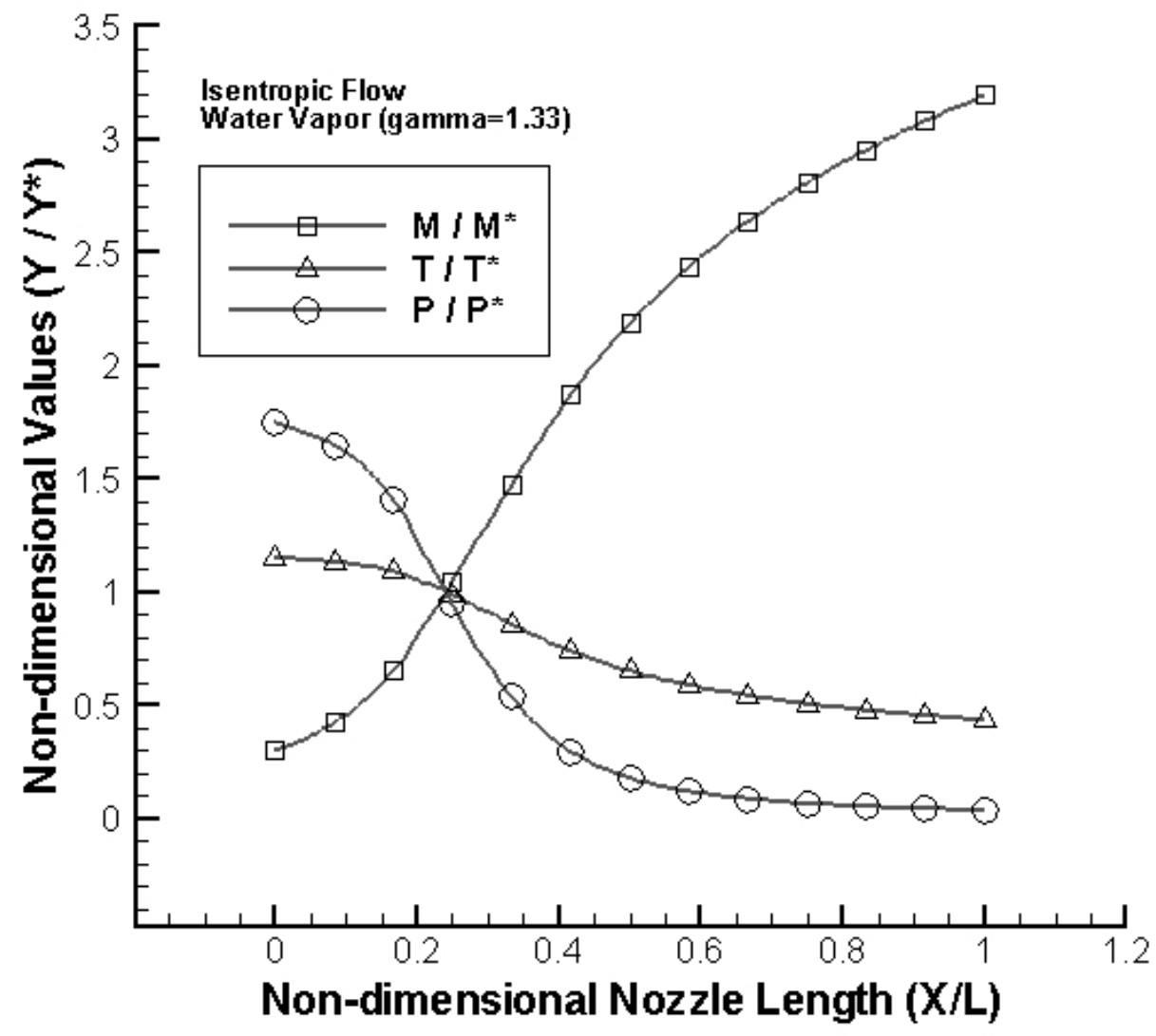

Figure 10: Non-dimensional values of Mach number, temperature, and pressure for isentropic flow along the nozzle.

As shown in Fig.9, the Mach number increases in the converging, reaches unity, and again increases in the diverging section. Conversely, both of temperature and pressure decrease in the both convergence and divergence sections. The results are in good agreement with either the equations of isentropic flow or the values of isentropic tables for $\gamma=1.33$ [29].

Second, the case of a flow in an adiabatic constant area duct in the existence of friction is studied. As to this case, the variation of area, stagnation temperature, and mass addition in Eq. (39) can be neglected. A sample circular duct of diameter $1 \mathrm{~cm}$ is assumed through which air $(\gamma=1.4)$ is flowing. The flow is assumed to enter the 
duct at a Mach number of 0.2. Also, a friction coefficient of $(f=0.02)$ is assumed to be between the flow and the surface of the duct. Since the flow enters the duct in subsonic condition, according to the Fanno line, its velocity (and Mach number) increases as it moves along the duct [29]. This increase can be continued until the flow reaches the sonic velocity [29].The important parameter of the flow, when there is friction in the duct, is ( $\mathrm{L}_{\max } / \mathrm{D}$ ), which is a function of $\gamma$ and Mach number. The parameter $\mathrm{L}_{\max }$ is the total length of the duct needed to increase the velocity of the flow to the sonic speed. Therefore, at the duct inlet, from the Fanno table, it can be obtained that [29]

$$
M_{1}=0.2 \rightarrow\left(\frac{f L_{\max }}{D}\right)_{1}=14.5333
$$

Thus, for instance, it can be concluded that if the duct length $7.0213 \mathrm{~m}$, then

$$
\left(\frac{f L_{\max }}{D}\right)_{2}=\left(\frac{f L_{\max }}{D}\right)_{1}-\frac{f L_{\text {total }}}{D}=14.5333-\frac{(0.02)(7.0213)}{0.01}=14.0426
$$

This parameter represents (fL/D) at the duct exit which can be used to find the Mach number at the exit. Therefore it can be obtained that

$$
\left(\frac{f L_{\max }}{D}\right)_{2}=14.0426 \rightarrow M_{2}=0.6
$$

Also, it can be demonstrated that if the duct length is $7.2666 \mathrm{~m}$, the Mach number at the exit equals one. To solve these cases numerically with the specified method, Eq. (39) can be rearranged as

$$
\frac{d M}{d x}=\frac{\psi M}{1-M^{2}}\left[\left(\frac{\gamma M^{2}}{2}\right)\left(\frac{4 f}{D_{h}}\right)\right]
$$


After initiating the problem regarding the above case, the grid size is chosen to be uniform 121 nodes. The Mach number distribution for the case of the duct of 7.2666 $\mathrm{m}$ is illustrated in Fig.10. It can be found out from this figure that the Mach number at $\mathrm{L}_{1}=7.0213 \mathrm{~m}$ and $\mathrm{L}_{2}=7.2666 \mathrm{~m}$, are about 0.6 and 1.0, respectively. The curve demonstrated in Fig.10 is the subsonic part of the Fanno line [29].

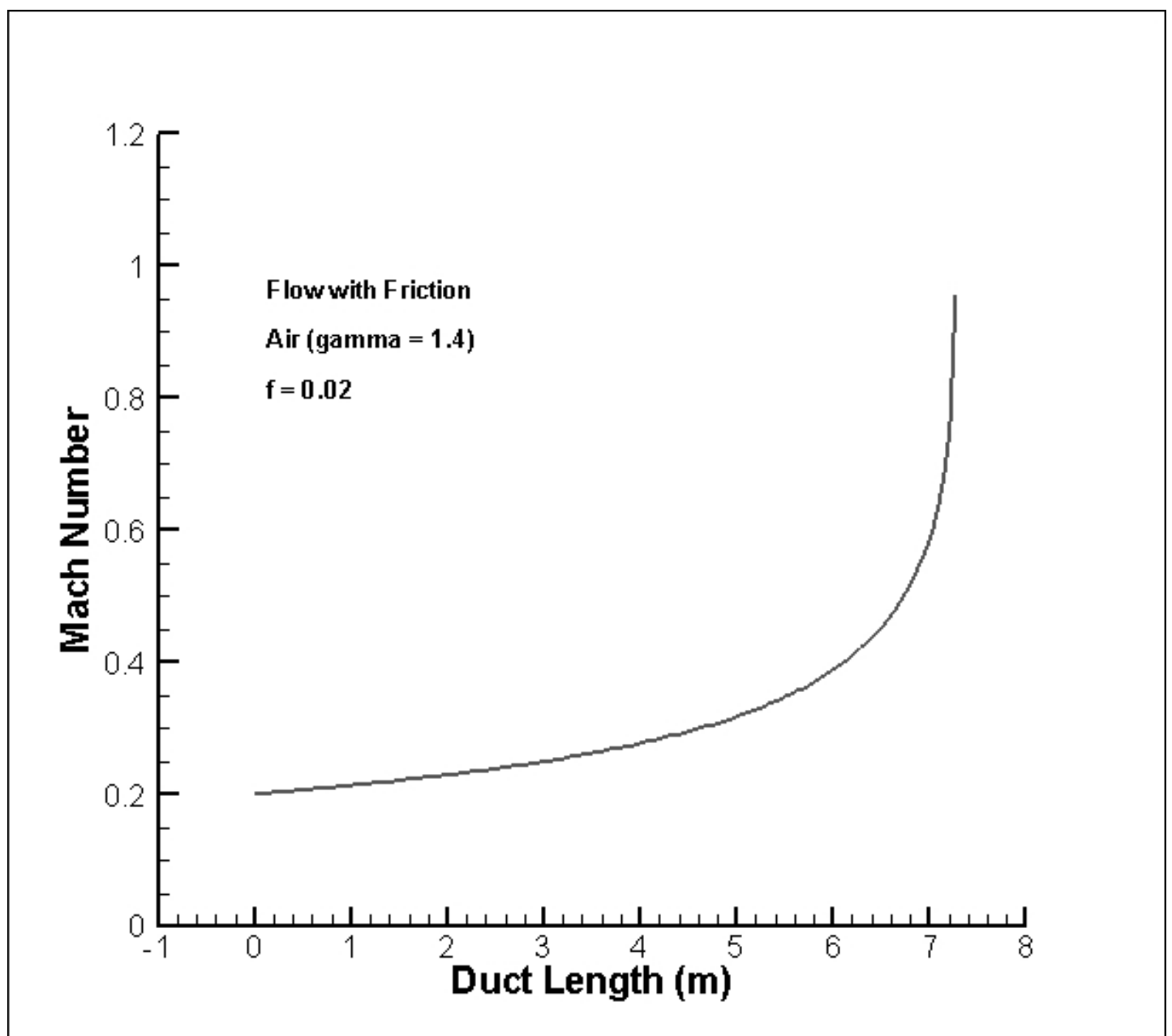

Figure 11: Mach number distribution of a flow with friction in an adiabatic constant area duct.

Finally, a case of a frictionless flow through a constant area duct is considered while the duct is subjected to a constant heat addition. Air $(\gamma=1.4, \mathrm{R}=287 \mathrm{~J} / \mathrm{kg} . \mathrm{K})$ is flowing through the duct, where a constant heat at a rate of $30 \mathrm{~kJ} / \mathrm{kg} \cdot \mathrm{m}$ is added. The 
inlet Mach number and temperature of the flow are 0.2 and $300 \mathrm{~K}$, respectively. According to the Rayleigh line, since the subsonic flow enters the duct, heat addition increases the velocity of the flow and thus the Mach number [29]. This velocity increase, without changing the mass flow rate, continues until the flow is sonic at the end of the duct [29]. Therefore, the maximum length of the duct in this case can be calculated as following. The stagnation temperature can be calculated from the isentropic tables as

$M_{1}=0.2 \rightarrow T_{t, 1}=302.3889 \mathrm{~K}$

Then, from the Rayleigh line, the maximum stagnation point can be obtained as $\mathrm{T}_{\mathrm{t}, 1}{ }^{\mathrm{N}}$ $=1741.871 \mathrm{~K}$. This increase in the stagnation temperature is because of the heat addition. Therefore, it is appropriate to write

$q=\bar{q} \cdot L_{\max }=c_{p}\left(T_{t, 1}^{*}-T_{t, 1}\right)=\frac{\gamma R}{\gamma-1} \cdot\left(T_{t, 1}^{*}-T_{t, 1}\right)$

Therefore, the maximum length can be calculated from above as $\mathrm{L}_{\max }=48.1987$.

In order to solve this case with the explained numerical methods, Eq. (39) needs to be simplified as

$\frac{d M}{d x}=\frac{\psi M}{1-M^{2}}\left[\frac{\left(1+\gamma M^{2}\right)}{2 T_{t}} \frac{d T_{t}}{d x}\right]$

In the equation above, the term of stagnation temperature can be expressed from Eq. (37) as

$\frac{d T_{t}}{d x}=\frac{\frac{T_{t}}{\psi} \cdot\left(\frac{\delta Q}{C_{p} \cdot T}\right)}{\Delta x}$

Setting a uniform grid size of 121 nodes and the given initial conditions, the Mach number distribution can be achieved. Figure.11 demonstrates the Mach number 
distribution along the duct. The Much number at the end of the duct can be obtained to be about one. The curve in Fig.11 is the subsonic part of the Rayleigh line [29].

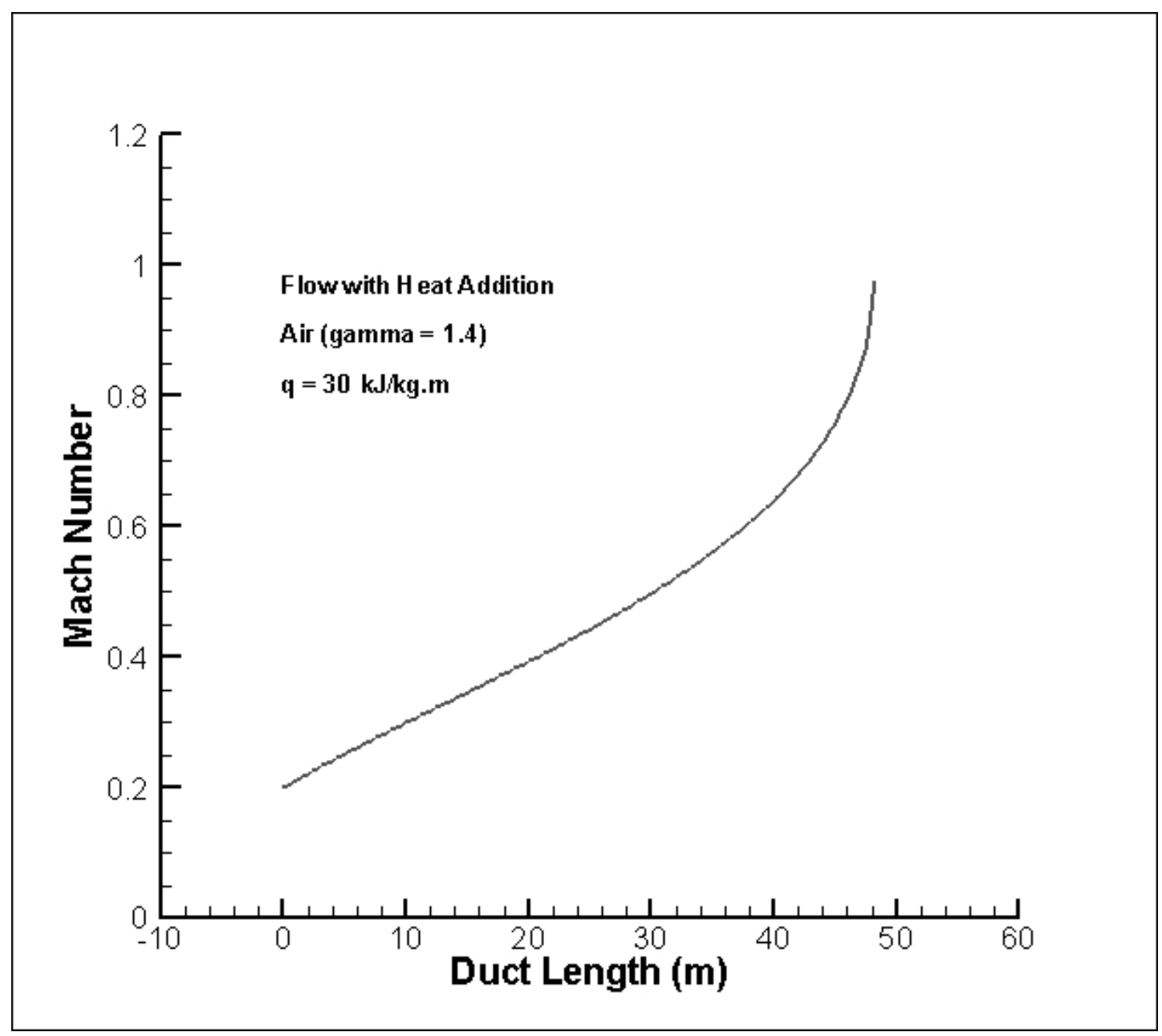

Figure 12: Mach number distribution of a flow with heat addition in a frictionless constant area duct. 


\section{Chapter 5}

\section{ACCURACY AND SENSITIVITY}

\subsection{Grid Sensitivity}

A grid sensitivity analysis shows the effect of grid size on the accuracy of the results.

It is assumed that the accuracy of the results rises as the number of grid points increases indicating that the most accurate results are those with the highest grid points. Both explicit and implicit models are tested to obtain the steady situation, and the results are then expressed and compared for five different grid points applied to the radial distance as $6,11,21$, and 31 nodes. A Si-C sample is assumed to have the thickness of $6 \mathrm{~cm}$ and porosity of $\varepsilon=50 \%$ through which Hydrogen flows at a rate of $5 \mathrm{gr} / \mathrm{s}$ as the coolant. The heat flux of $5000 \mathrm{~W} / \mathrm{m}^{2}$ is applied to the hot face, and the cold face pressure and temperature are set to $1.35 \mathrm{MPa}$ and $290 \mathrm{~K}$, respectively. Figures.12 and 13 illustrate the temperature distributions for these nodes for explicit and implicit methods. An identical time step equal to 0.1 (i.e. $\Delta \mathrm{t}=0.1$ ) is applied to all the grid points. 


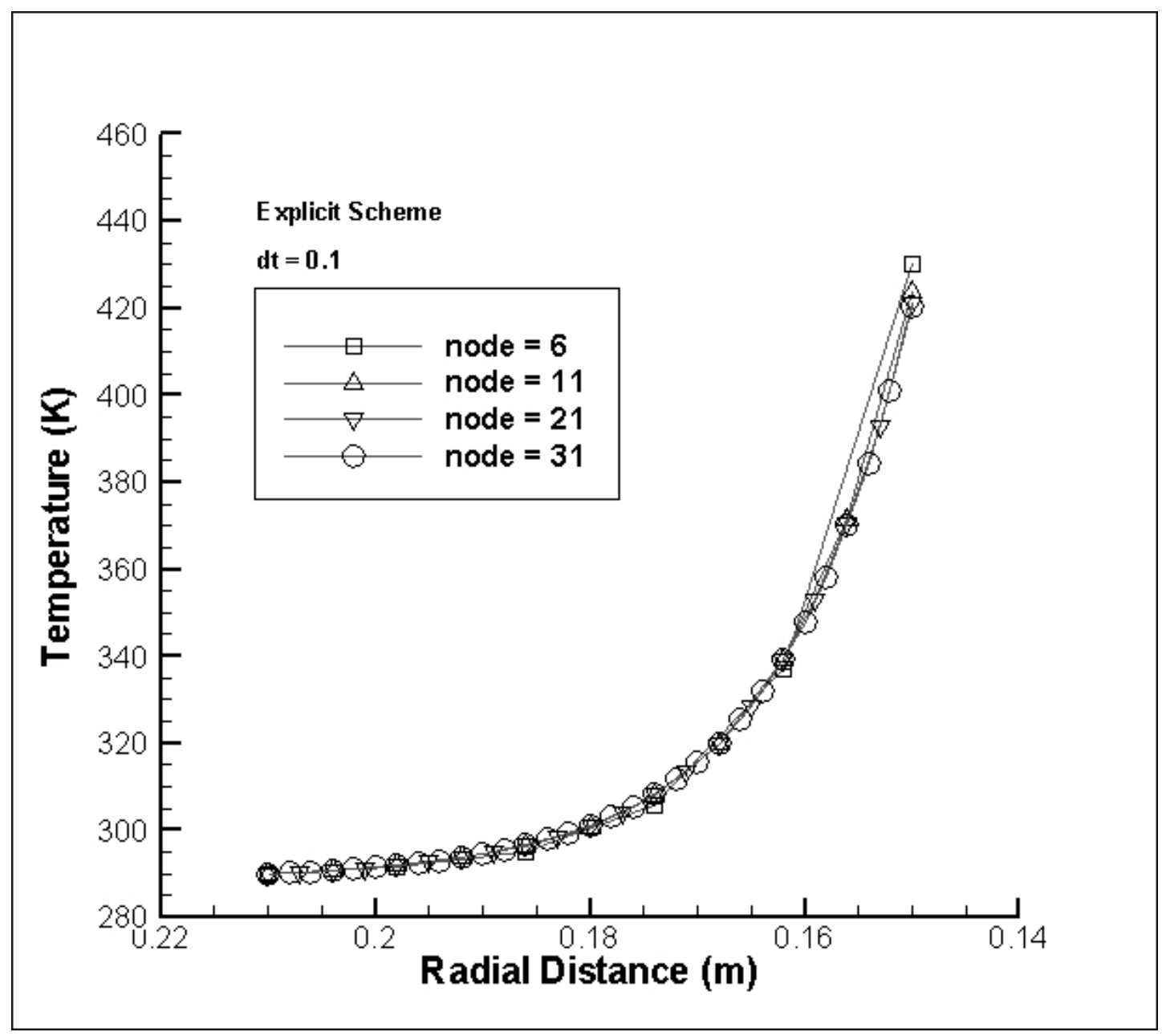

Figure 13: Temperature profile and grid sensitivity of explicit scheme for different nodes of $6,11,21$, and 31 and $\mathrm{dt}=0.1$. 


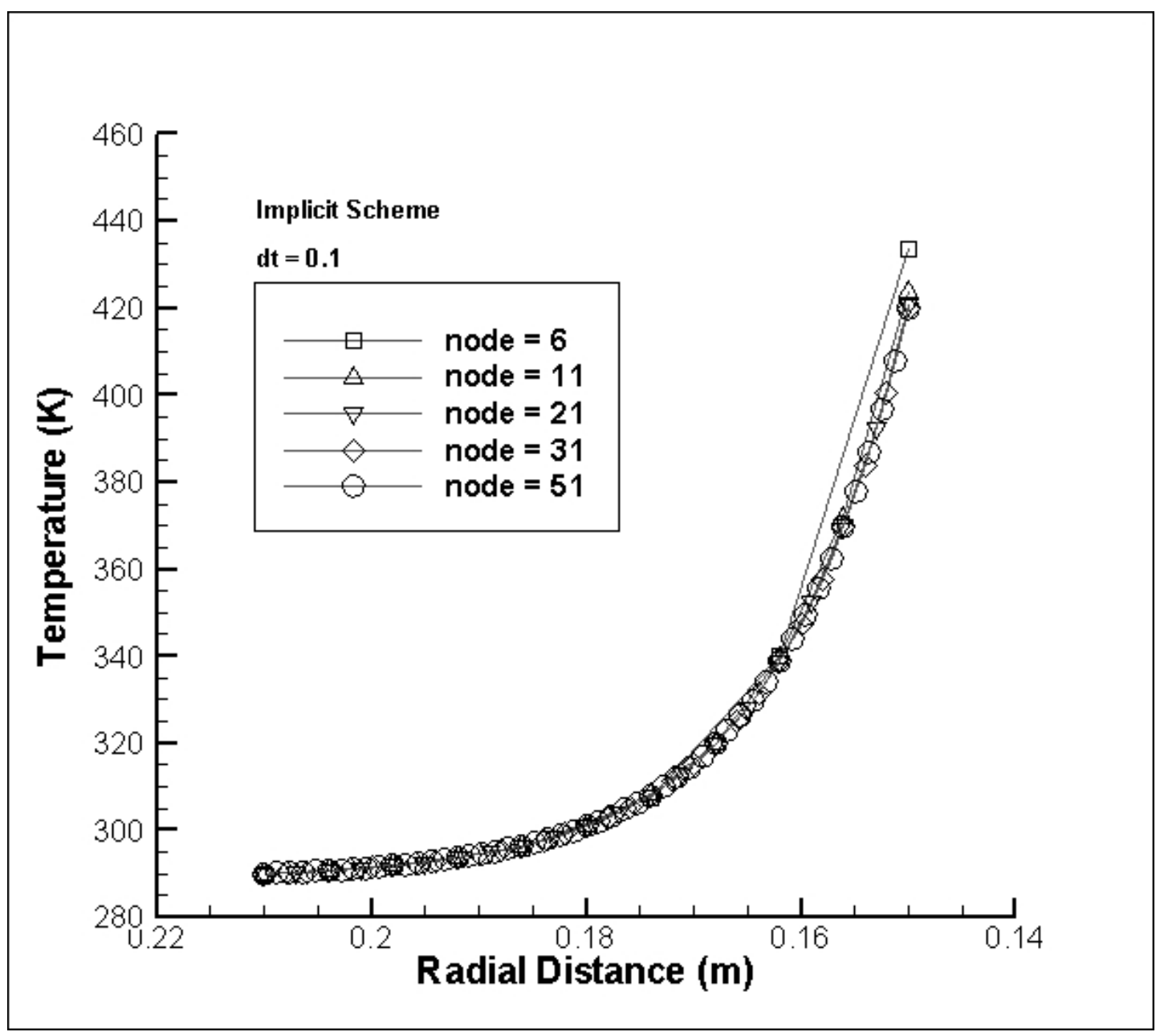

Figure 14: Temperature profile and grid sensitivity of implicit scheme for different nodes of $6,11,21$, and 31 and $\mathrm{dt}=0.1$.

Acceptable accuracies are gained with respect to the grid changes for both schemes. As both models show, more deviation in the temperature profile appears for the case of 6 nodes, and for larger number of nodes the results become closer. As these figures show, the results of both numerical schemes are very similar because the discretization methods applied to the both models are of second order in space. Also, an error analysis is developed compared to the hot face temperature of the finest grid (i.e. 31 nodes) in order to clarify the grid dependency of both models (i.e. Tables 3, 4). The errors presented in Tables 3 and 4 are relative errors and were calculated as

$$
\text { error }=\frac{\mathrm{T}-\mathrm{T}_{31}}{\mathrm{~T}_{31}} \times 100
$$


where $\mathrm{T}$ and $\mathrm{T}_{31}$ are the hot face temperatures of each case and the case with 31 nodes, respectively.

Table 3: Relative error analysis of hot face temperature for explicit method for grid

sensitivity $\left(\right.$ error $\left.=\frac{\mathbf{T}-\mathbf{T}_{\mathbf{3 1}}}{\mathbf{T}_{\mathbf{3 1}}} \times \mathbf{1 0 0}\right)$.
\begin{tabular}{|c|c|c|}
\hline Node & Hot Face Temp. & Error(\%) \\
\hline 6 & 430.03 & 2.3 \\
\hline 11 & 423.64 & 0.76 \\
\hline 21 & 421.03 & 0.14 \\
\hline 31 & 420.43 & - \\
\hline
\end{tabular}

Table 4: Relative error analysis of hot face temperature for Implicit Method for grid

\begin{tabular}{|c|c|c|}
\hline Node & Hot Face Temp. & Error(\%) \\
\hline 6 & 433.63 & 3.24 \\
\hline 11 & 423.92 & 0.93 \\
\hline 21 & 420.65 & 0.15 \\
\hline 31 & 420.01 & - \\
\hline
\end{tabular}

\subsection{Time Step Sensitivity}

The size of the time step in the explicit method must be selected so that the stability criterion is satisfied. Furthermore, different time steps will cause different accuracies in the results depending on the applied numerical method. To observe the time step sensitivity of the models, similar porous sample type, coolant and boundary conditions to the section 4.5 are defined and applied to the codes. Figures.14 and 15 illustrate the temperature comparison of the both methods for six different time steps 
of 5.0, 1.0, 0.2, 0.04, 0.008 and 0.0016. All of these time steps are applied to a model with the grid points of 21 (i.e. node $=21$ ).

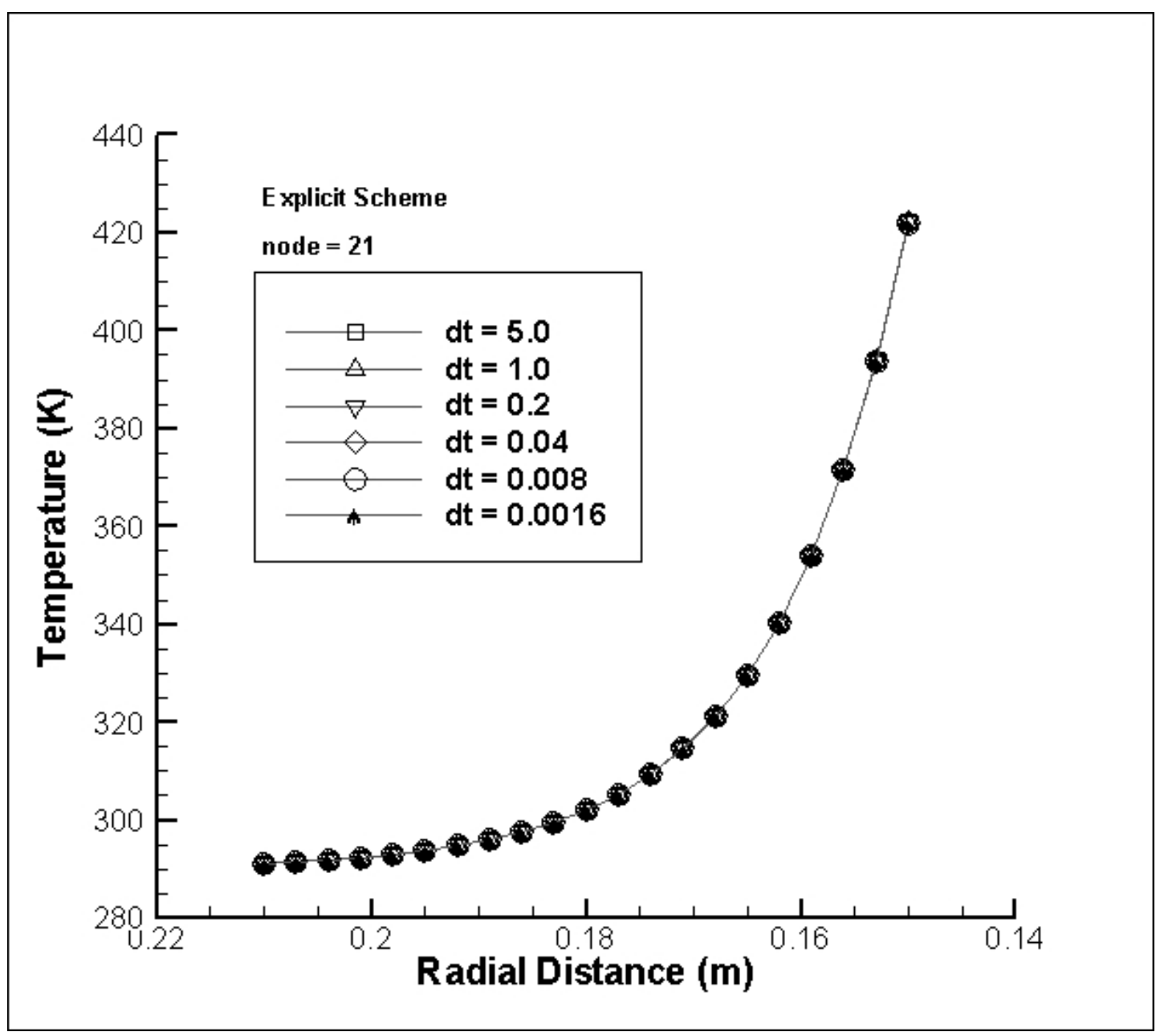

Figure 15: Temperature profile and time step sensitivity of explicit scheme for different time steps of 5.0, 1.0, 0.2, 0.04, 0.008 and 0.0016 and 21 nodes. 


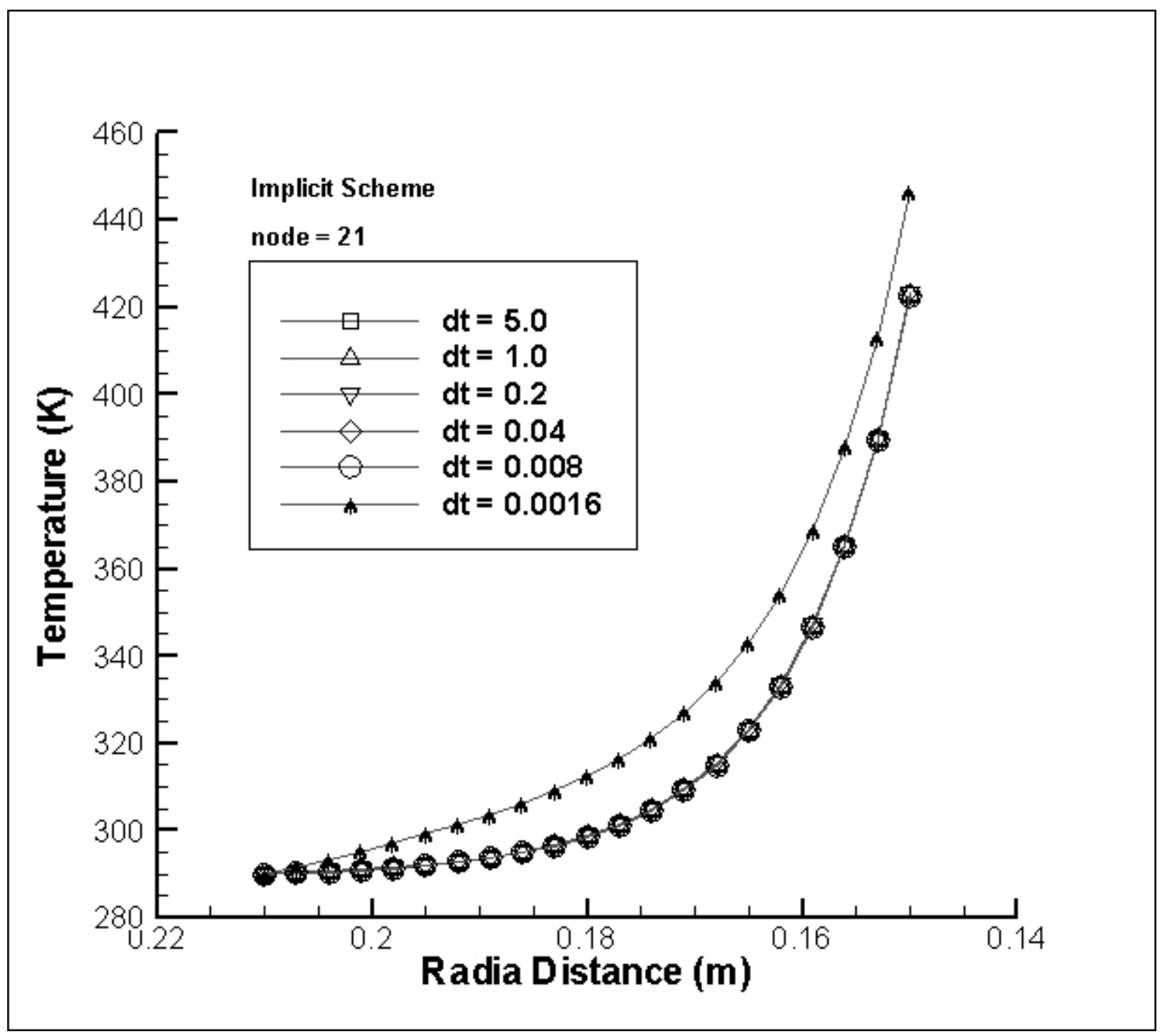

Figure 16: Temperature profile and time step sensitivity of implicit scheme model for different time steps of 5.0, 1.0, 0.2, 0.04, 0.008 and 0.0016 and 21 nodes.

The results of the explicit model indicate smaller changes, as the time step decreases, which imply more accurate and stable results for a wide range of time steps. As to the implicit model, the results remain almost constant as the time step narrows except for the case of $\Delta t=0.0016$. Thus, for the lower time steps, the accuracy of the implicit method decreases. It can also be concluded that the implicit model is more sensitive than the explicit scheme, whereas, the explicit model reveals more reliable results. 
The running time of each code varies according to the grid size and the time step chosen in each solution. As a test, both implicit and explicit codes are run for the specified time steps and 21 nodes. Table 5 shows the running time of each code for the time steps of 5.0, 1.0, 0.2, 0.04, 0.008 and 0.0016 .

Table 5: Running time for each model (in seconds) for different time steps using 21

\begin{tabular}{c|cccccc}
\multicolumn{7}{c}{ nodes. } \\
\hline Implicit & $\Delta \boldsymbol{t}=\mathbf{5 . 0}$ & $\mathbf{1 . 0}$ & $\mathbf{0 . 2}$ & $\mathbf{0 . 0 4}$ & $\mathbf{0 . 0 0 8}$ & $\mathbf{0 . 0 0 1 6}$ \\
Method & 0.15 & 0.9 & 3.4 & 14.9 & 55.3 & 233.2 \\
\hline Explicit & 0.06 & 0.3 & 1.01 & 4.36 & 17.42 & 67.31 \\
Method & & & & & & \\
\hline
\end{tabular}

The explicit code shows about three times faster runs for all the time steps than the implicit code.

\subsection{Gas Flow Equations}

As previously expressed, a $4^{\text {th }}$ order Runge-Kutta method was applied to solve the differential equation of Mach number distribution. The stability analysis of a $4^{\text {th }}$ order Runge-Kutta method was discussed elaborately in the literature [30]. The solution method of the differential equation of Mach number (i.e. Eq. (39)) was presented earlier can generally be expressed as

$M_{i+1}=M_{i}+d x_{i}\left(\sum_{j=1}^{j=4} b_{j} k_{i, j}\right)$

where, the values of $b_{j}$ and $k_{i, j}$ are weights and stage values, respectively. Difficulties occur in obtaining the criterion of stability of the Runge-Kutta method due to the fact that the Mach number distribution in Eq. (39) is a multivariable function. Another 
method of analyzing the stability of this method can be studying the grid sensitivity of it. Grid sensitivity reveals whether or not the solution remains within its range of stability and consistency. However, it shows the domain of accuracy of the numerical solution.

\subsection{Grid Sensitivity}

As well as equations of porous media, a sample problem is specified to be solved in order to demonstrate the grid sensitivity of the Runge-Kutta method which is applied to the gas dynamic equations in the thrust chamber. Then, the Mach number distribution for different grid sizes is to be achieved from the solution. The sample nozzle of length $2 \mathrm{~m}$ and given profile, explained in section 3, is considered through which the hot gases flow in an isentropic manner. Uniform grids of $31,41,51,81$, 121, and 201 nodes are applied to the model and the flow initial conditions (i.e. flow properties of the nozzle inlet) are provided in section 3 as well. Figures.16 and 17 illustrate the Mach number profile for the specified grid points. 


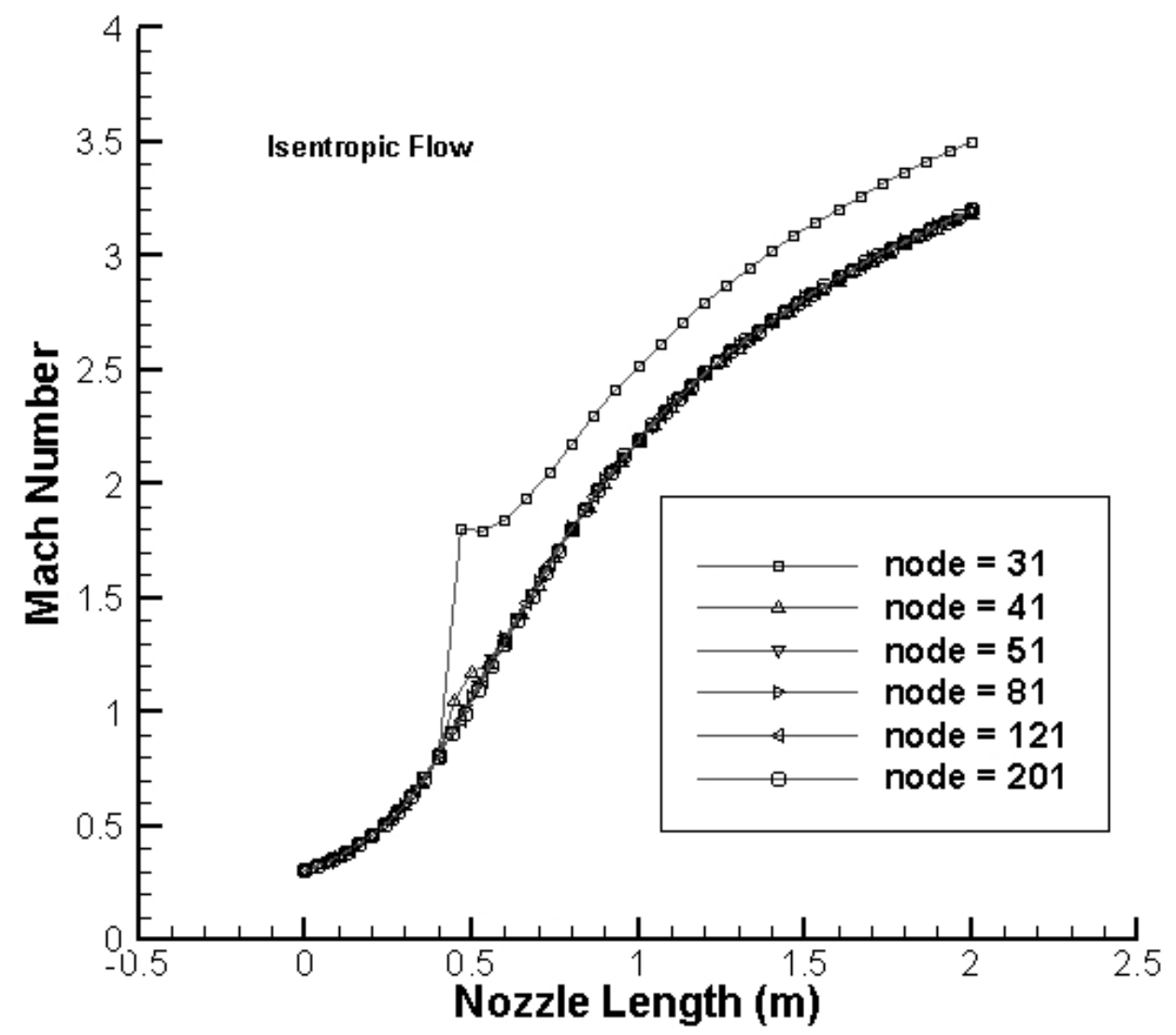

Figure 17: Mach number profile and grid sensitivity for different nodes of 31, 41, 51, 81, 121, and 201. 


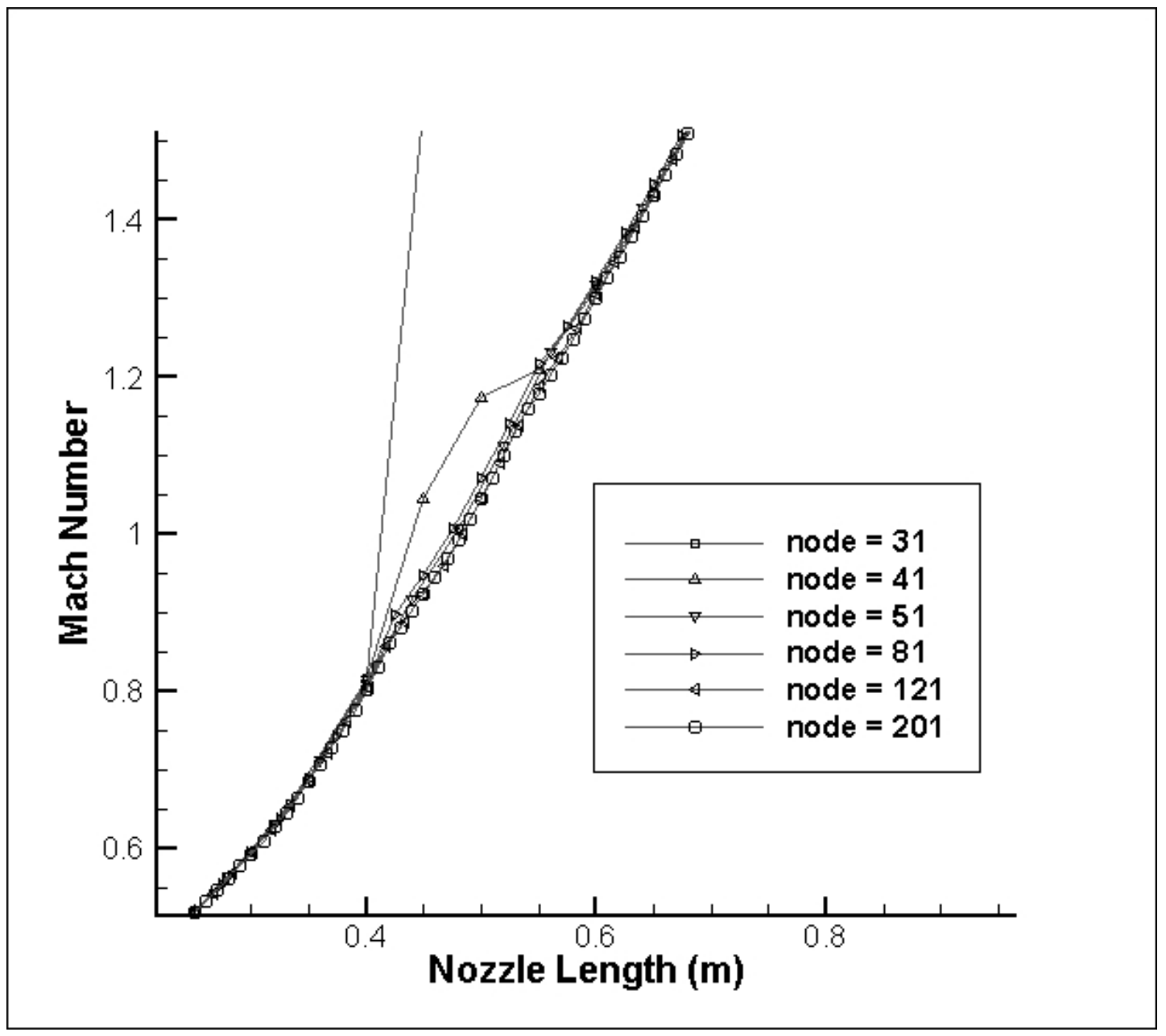

Figure 18: Mach number profile at the throat (i.e. $\mathrm{x}=0.5 \mathrm{~m}$ ) for different nodes of 31, 41, 51, 81, 121, and 201.

As it is shown in these figures, there are some irregularities about the nozzle throat where the Mach number approaches unity for a coarse grid size (i.e. 31 and 41 nodes). For these cases, the solution still remains stable but inaccurate, and for lower number of nodes, the solution diverges. On the other hand, as the number of nodes rises, the Mach number distributions show acceptable accuracies. However the Mach number values are very close for higher number of nodes, Fig.17 focuses on the Mach number of the throat region and demonstrates these differences more clearly. Therefore, the values of Mach number at the throat for different number of 
nodes and also the generated error, regarding the finest grid (i.e. 201 nodes), are tabulated and shown in Table 6.

Table 6: Mach number values at the throat and the relative error (error $=$ $\left.\frac{M_{\text {th }}-M_{\text {th,201 }}}{M_{\text {th,201 }}} \times 100\right)$.

\begin{tabular}{|c|c|c|}
\hline Node & Mach Number at Throat & Error(\%) \\
\hline $\mathbf{3 1}$ & 1.780 & 77 \\
\hline $\mathbf{4 1}$ & 1.040 & 3.89 \\
\hline $\mathbf{5 1}$ & 1.006 & 0.49 \\
\hline $\mathbf{8 1}$ & 1.005 & 0.39 \\
\hline $\mathbf{1 2 1}$ & 1.002 & 0.09 \\
\hline $\mathbf{2 0 1}$ & 1.001 & - \\
\hline
\end{tabular}




\section{Chapter 6}

\section{RESULTS AND CASE STUDY}

Parametric studies are developed in order to clarify the main factors dealing with transpiration cooling and also the importance of them during this process. As mentioned earlier, one of the significance of parametric studies on transpiration cooling process is determining and calculating the parameters which cannot directly be measured in experiments, such as heat flux within the porous layer. However, in some cases there are equipment specifically designed to measure a special parameter, but the range of that parameter is much far from the designed range of the device. Moreover, a wider range of variable effects on this process can be studied using a beneficial parametric study. In this section, first, a complete analysis is carried out on the main parameters within the porous medium, which have important roles on the cooling process. Subsequently, a comprehensive coupled model of transpiration cooling, considering the interactions between porous media and thrust chamber gases is presented.

\subsection{Flow in Porous Medium}

In this section effect of four parameters are studied: coolant mass flow rate, coefficient of heat transfer, coolant gas type and the sample thickness. First, assuming hydrogen as the coolant, transpiration cooling is considered and solved for different mass flow rates of it. A heat transfer analysis is also carried out which can illustrate the effective heat transfer mechanisms during transpiration cooling within the sample. Different boundary conditions of constant gas temperature and heat 
transfer coefficient at the hot face are considered, so that the temperature change at the hot and cold faces can be observed. Also, the state of thermal equilibrium between solid and fluid is assumed and energy equation (i.e. Eq. (3)) is solved. Second, transpiration cooling in the sample is modeled for different gases such as Air, Helium and Hydrogen to show the cooling capacity of each gas. Finally, the cooling process is simulated for different sample lengths so that the effect of porous thickness can be monitored.

\subsection{Effect of Coolant Mass Flow Rate}

The sample is assumed to be a $\mathrm{Si}-\mathrm{C}$ porous material having a thickness of $6 \mathrm{~cm}$. The properties of the sample are presented in Table 7.

Table 7: Properties of Si-C porous material.

\section{Si-C Porous}

Property

\section{Material}

\begin{tabular}{cc}
\hline $\mathbf{L}[\mathbf{m}]$ & 0.06 \\
$\boldsymbol{\varepsilon}[\%]$ & 50 \\
$\mathbf{d}_{\mathbf{p}}[\mathbf{m}]$ & 0.000635 \\
$\mathbf{k}_{\mathbf{s}}[\mathbf{W} / \mathbf{m . K}]$ & 1.0 \\
$\mathbf{c p}_{\mathbf{s}}[\mathbf{J} / \mathbf{k g . K}]$ & 1422.6 \\
\hline
\end{tabular}

The transpiration cooling problem is solved considering six different mass flow rates of liquid Hydrogen as 0.0, 0.1, 0.5, and 1.0, 2.0, and $5.0 \mathrm{gr} / \mathrm{s}$. The coolant is injected from the cold face at a pressure of $1.35 \mathrm{MPa}$ and temperature of $290 \mathrm{~K}$. Also, hot gases, having the temperature of $800 \mathrm{~K}$, are in contact with the hot face, where the convective heat transfer coefficient is $100 \mathrm{~W} / \mathrm{m}^{2} . \mathrm{K}$. Uniform grids corresponding to 
21 nodes and time steps of 1.e-3 are applied to the code. Both the residual and relative errors are restricted by the order of $1 . e-7$ in order to obtain the successful stability and convergence. Figures.18 shows the temperature profile within the pors layer for six mass flow rates of the coolant, accordingly.

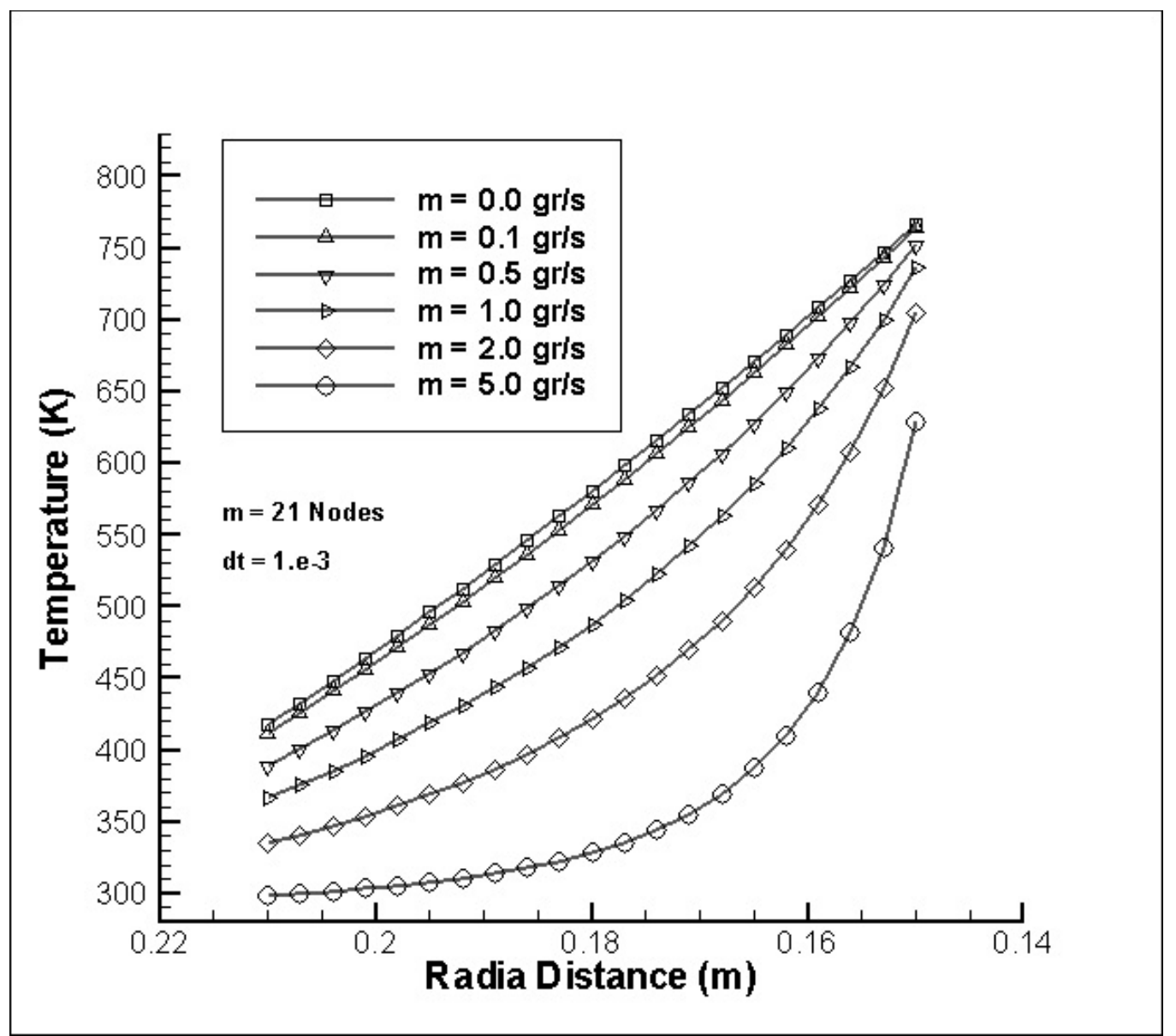

Figure 19: Temperature profile for different mass flow rates within the sample.

As to the lower mass flow rates, such as $0.0,0.1$, and even 0.5 , the convective term in the energy equation diminish and thus, the temperature distribution becomes almost linear. As the coolant flow rate rises, the temperature profiles form exponential distributions and the effect of cooling can be observed within the sample. As to the case of no cooling, (i.e. mass flow rate $=0$ ), the temperature reaches about $766 \mathrm{~K}$ at the hot face and $417 \mathrm{~K}$ at the cold face. Whereas, for the highest coolant 
flow rate, these temperatures are $629 \mathrm{~K}$ and $299 \mathrm{~K}$ at the hot and cold faces, respectively. The temperature of the hot and cold boundaries versus coolant flow rates are illustrated in Fig.19.

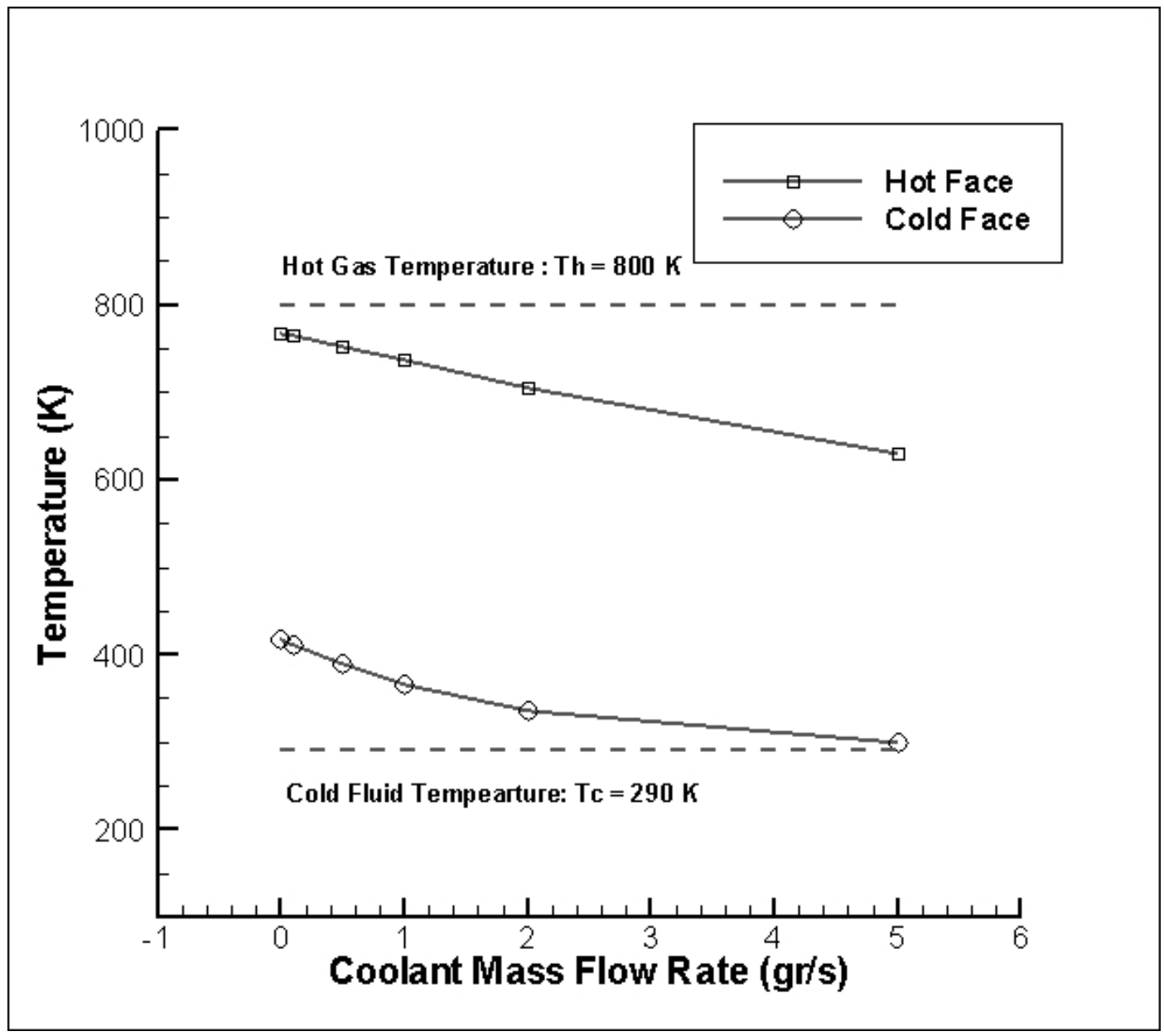

Figure 20: Temperature profile at the hot and cold faces for different mass flow rates.

Figure 20 demonstrates the absolute values of radial velocity of the coolant flow towards the hot face. As the cylindrical coordinates is defined from the hot face towards the cold face, and because of higher pressure applied to the cold face, the amounts of flow velocity have negative values. The important point is that the negative velocity values make the convective term of the energy equation negative, which demonstrates the contradiction of the convective and conductive heat transfer terms. As explained before, the velocity domain is obtained from the solution of 
continuity equation. Regarding Fig.20, for lower values of mass flow rate, the velocity profile is almost linear, while, as the flow rate rises, the velocity profile becomes a curve. This can be due to the decrease in the side area $(A=2 \pi r)$ of the porous layer and also, variation of density as it is predicted to decrease because of temperature rise. That is, for higher values of flow rate, the effect of density in the continuity equation reduces and thus, the velocity contribution in order to maintain a constant mass flow rate increases.

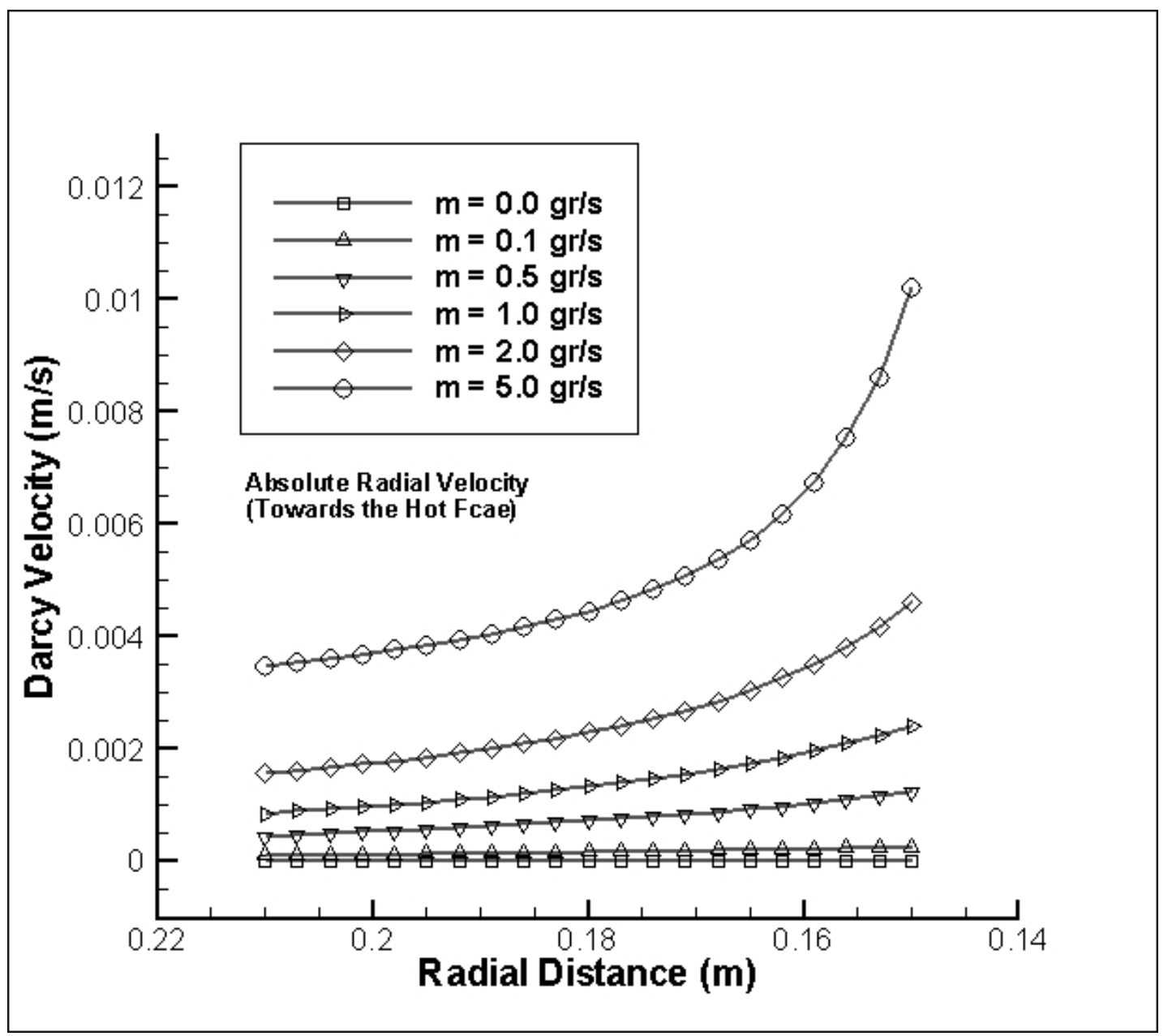

Figure 21: Radial velocity profile the coolant flow towards the hot face for different coolant flow rates within the sample. 
Also, density of the coolant is calculated from the equation of state and illustrated in Fig.21. Since density of a perfect gas varies due to the temperature and pressure changes, it is predictable that the density of the coolant decreases as it flows towards the hot face for a constant mass flow rate. According to Fig.21, density of the coolant has its maximum of $0.784 \mathrm{~kg} / \mathrm{m}^{3}$ at the cold face, and minimum of $0.427 \mathrm{~kg} / \mathrm{m}^{3}$ at the hot face for the case of no coolant mass flow rate. As the flow rate increases, the range of density values increases due to the temperature change and reaches to the maximum of $1.094 \mathrm{~kg} / \mathrm{m}^{3}$ at the cold face and $0.527 \mathrm{~kg} / \mathrm{m}^{3}$ at the hot face for the case of $5.0 \mathrm{gr} / \mathrm{s}$. Also, according to the radial velocity range, the Reynolds number based on the average pore diameter of $0.635 \mathrm{~mm}$, is determined for the model ( $R e=$ $\left.\frac{\rho|U| D_{p}}{\mu}\right)$. It is within the range of 0.04 and 0.18 , which implies that the flow regime is clearly laminar and thus the Darcy's law is applicable. 


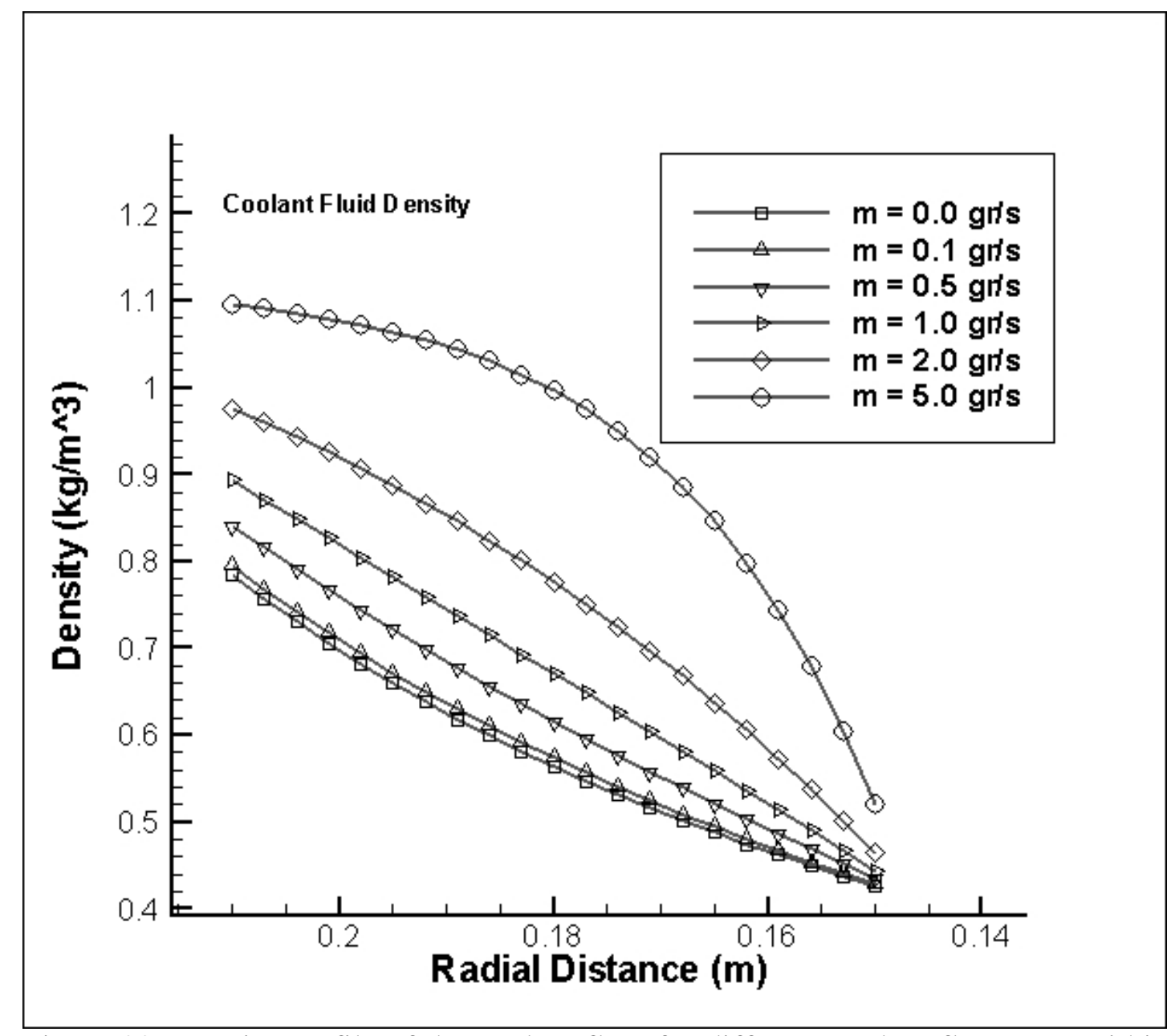

Figure 22: Density profile of the coolant flow for different coolant flow rates within the sample.

Heat flux within the porous media can also be calculated considering conduction and convection in the porous media as

$\dot{q}=-k_{A} \frac{d T}{d r}+\varepsilon_{A} \rho_{f} c_{p f} u_{f} T$

The heat flux at the hot face is equal to the convective heat flux between hot face and the gas due to the hot face boundary condition. The profile of heat flux of the model for different mass flow rates of coolant was demonstrated in Fig.22. 


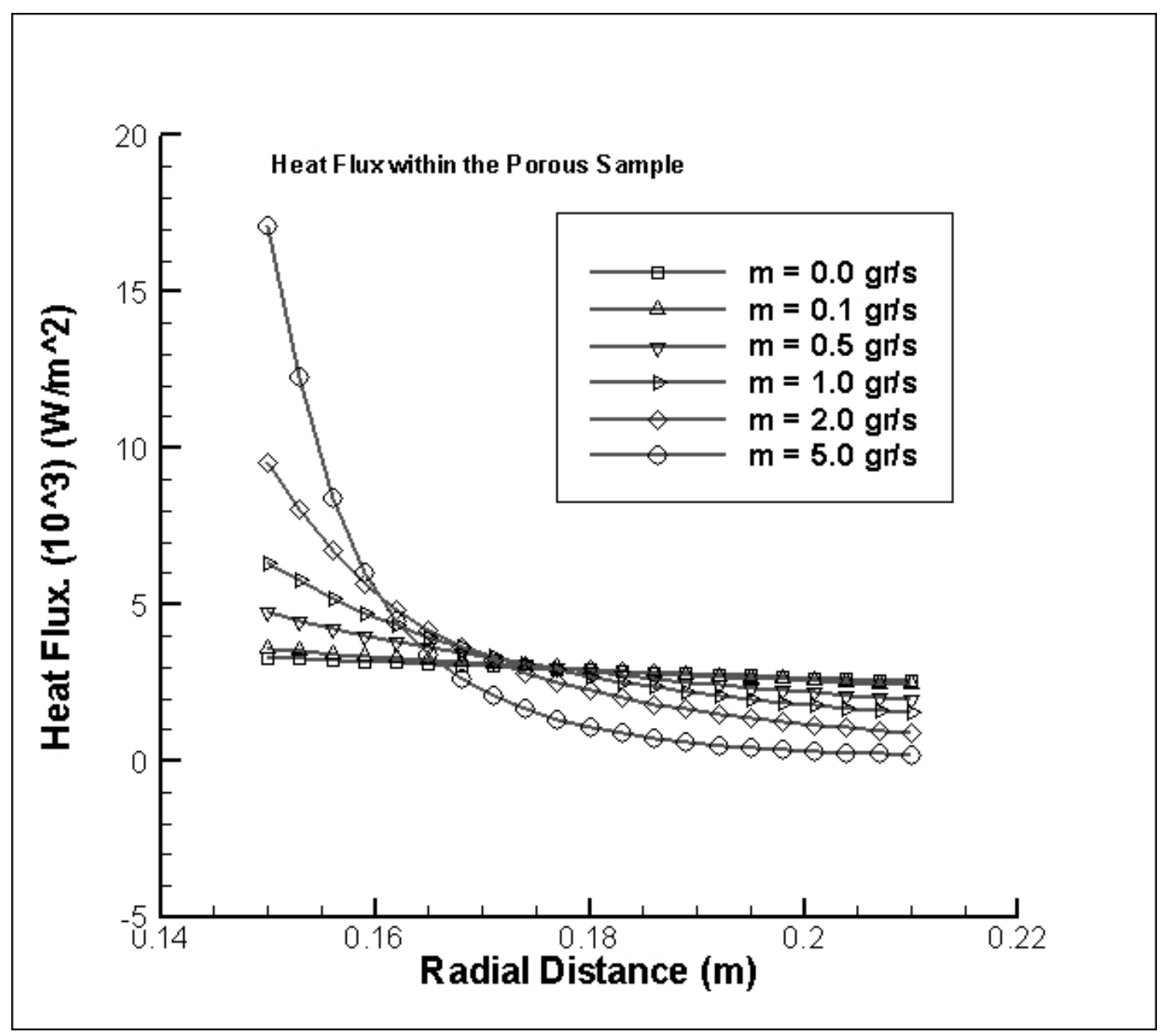

Figure 23: Heat flux distribution within the porous sample for different coolant mass flow rates.

As illustrated in this figure, the maximum and minimum heat fluxes occur at the hot and cold faces respectively. This reduction can be because of two issues: first, the effective area of heat transfer increases as the radius increases, and second, the temperature gradient decreases as the radius increases. Also, it can be observed that for the higher flow rates of coolant, the heat flux values are accumulated towards the hot face, while the values near the cold face diminish which implies the effectiveness of coolant flow through the sample.

\subsection{Cooling Effectiveness}


Figure.22 also shows the effect of coolant flow rate on the heat penetration into the sample. The heat-blocking effect of the transpiration cooling can be better observed by defining cooling effectiveness. This non-dimensional parameter can be expressed as the ratio of the net heat flux with cooling to that of without cooling as

$\epsilon=\frac{\text { Net Heat flux with transpiration cooling }}{\text { Net Heat flux without transpiration cooling }}=\frac{\left(\dot{q}_{\text {in }}-\dot{q}_{\text {out }}\right)_{\text {cooling }}}{\left(\dot{q}_{\text {in }}-\dot{q}_{\text {out }}\right)_{\text {no cooling }}}$

where the parameters $\dot{q}$ denote heat flux and can be obtained from Eq. (69). Also, the subscripts "in" and "out" denote the inner and outer faces of porous layer, respectively. Therefore, Eq. (70) can be calculated as

$\epsilon=\frac{\left[(\dot{q})_{r_{i}}-(\dot{q})_{r_{o}}\right] \text { for given coolant flow rates }}{\left[(\dot{q})_{r_{i}}-(\dot{q})_{r_{o}}\right] \text { for no coolant flow rate }}$

According to the definition above, the cooling effectiveness has its minimum value, which is "one" for the pure conduction case. It is predicted that this factor increases as the coolant flows through the sample and gets its maximum for the maximum coolant mass flow rate, thus maximum cooling effect. Therefore, the higher the coolant mass flow rate, the higher the cooling effectiveness. This parameter is calculated from the heat flux values (Fig.22) for different coolant mass flow rates (see Fig.23). 


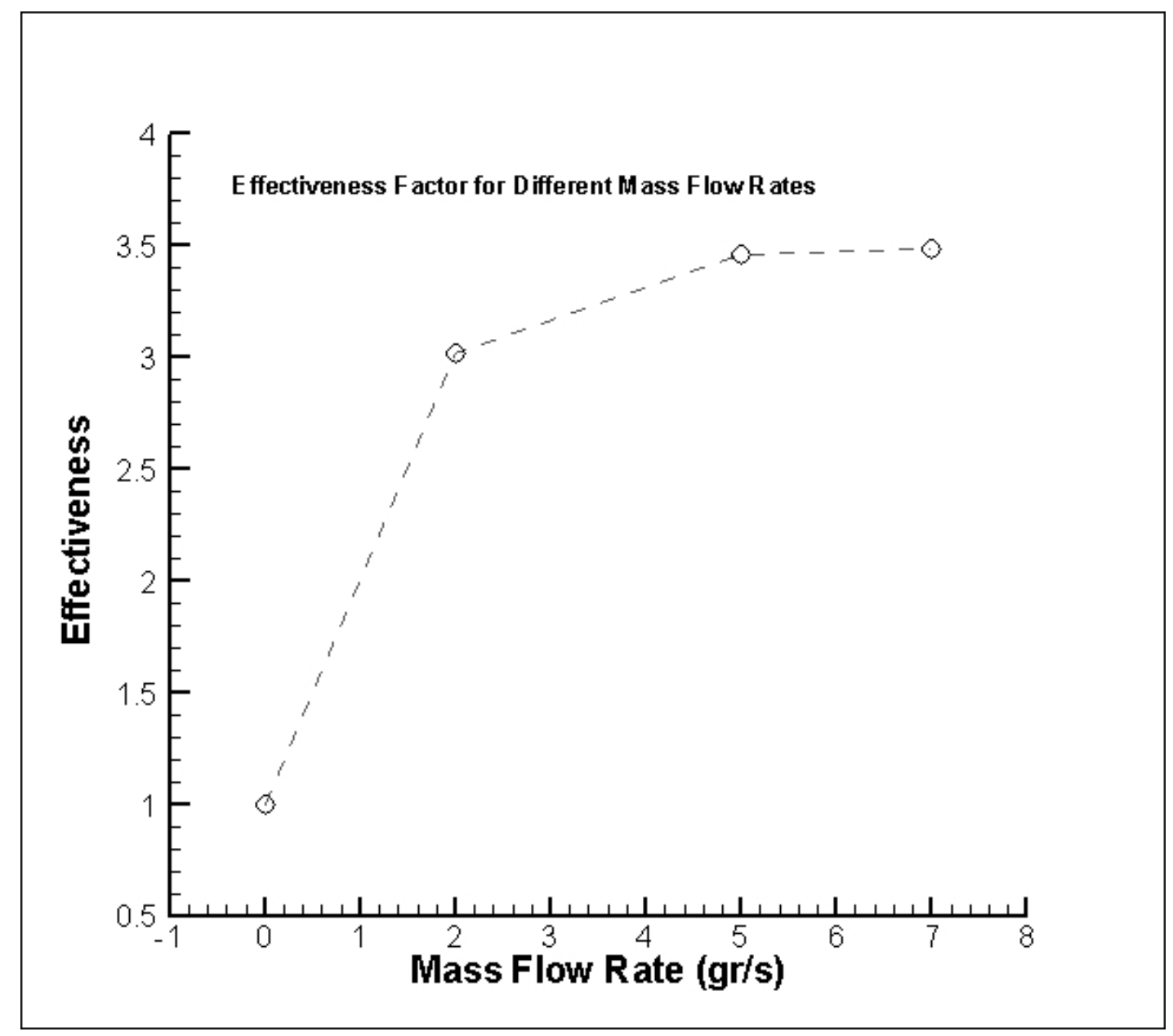

Figure 24: Effectiveness Factor for different mass flow rates.

According to this figure, the effectiveness factor is equal to one when the mass flow rate is $0.0 \mathrm{gr} / \mathrm{s}$, and it rises up as the mass flow rate increases. This factor for the mass flow rates of 2.0, 5.0, and 7.0 gr/s would be 3.07, 3.48, and 3.51, respectively. It can be concluded that the effectiveness factor approaches to a finite value as the coolant mass flow rate increases.

\subsection{Effect of Hot Boundary Condition}

During the transpiration cooling, two parameters at the hot face can remarkably interfere with this process: the coefficient of convective heat transfer at the hot boundary, and the hot gas temperature. In order to observe the effect of these parameters, the same porous sample is considered while, Hydrogen, as the coolant, 
flows at a rate of $7.0 \mathrm{gr} / \mathrm{s}$ through it. The coolant is injected from the same cold face condition. Two cases are considered with different hot face conditions: first, a hot gas temperature of $800 \mathrm{~K}$ with different coefficients of heat transfer of 100,500 , and $1000 \mathrm{~W} / \mathrm{m}^{2} . \mathrm{K}$. The results are arranged such that the temperature profiles within the sample can be observed. Second, the convective heat transfer of $300 \mathrm{~W} / \mathrm{m}^{2} . \mathrm{K}$ with the hot gas temperatures of 500, 700, and $900 \mathrm{~K}$. Figures.24 and 25 demonstrate the profiles of the first and second cases, respectively.

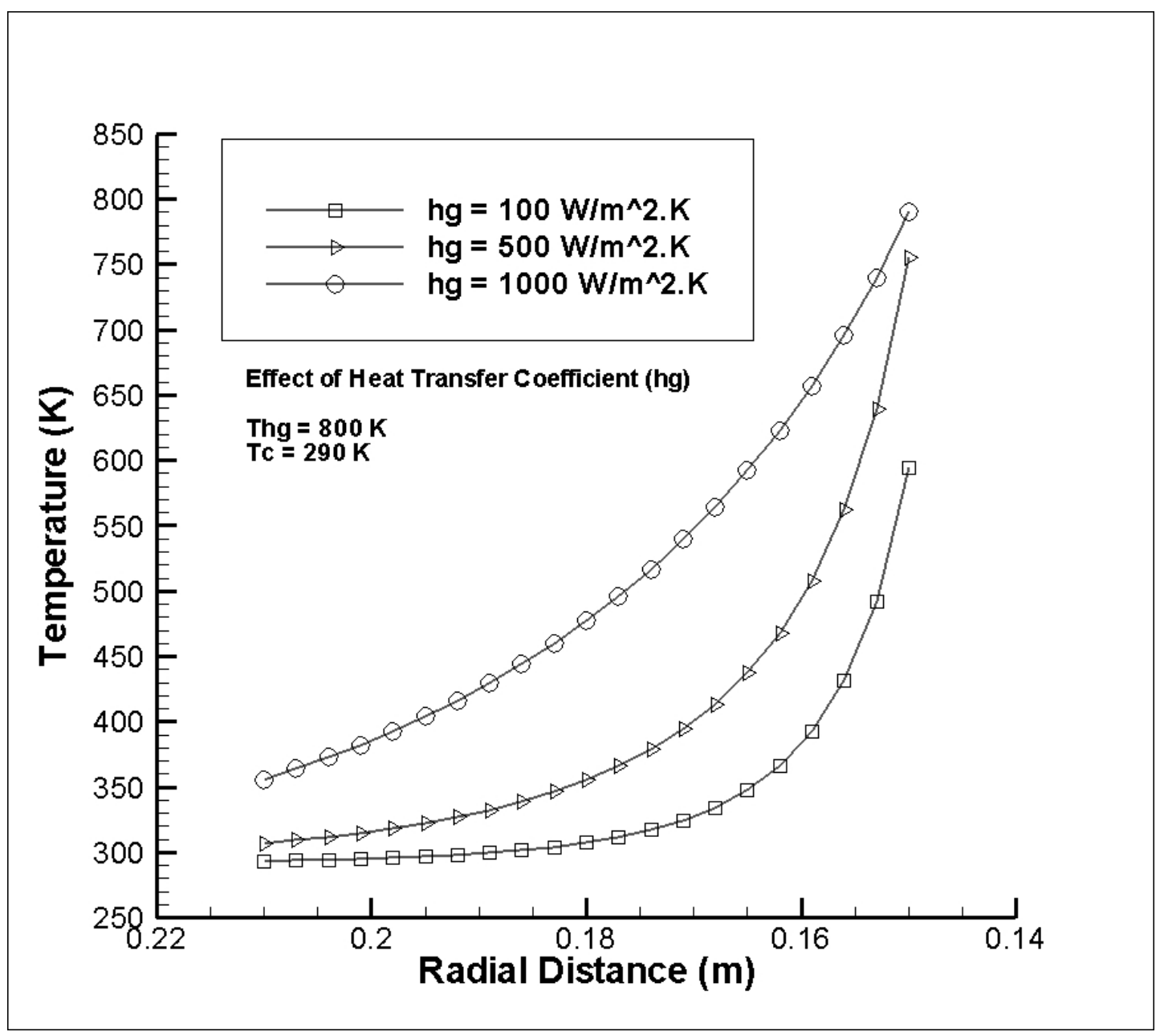

Figure 25: Effect of hot face heat transfer coefficient on temperature profile within the sample. 


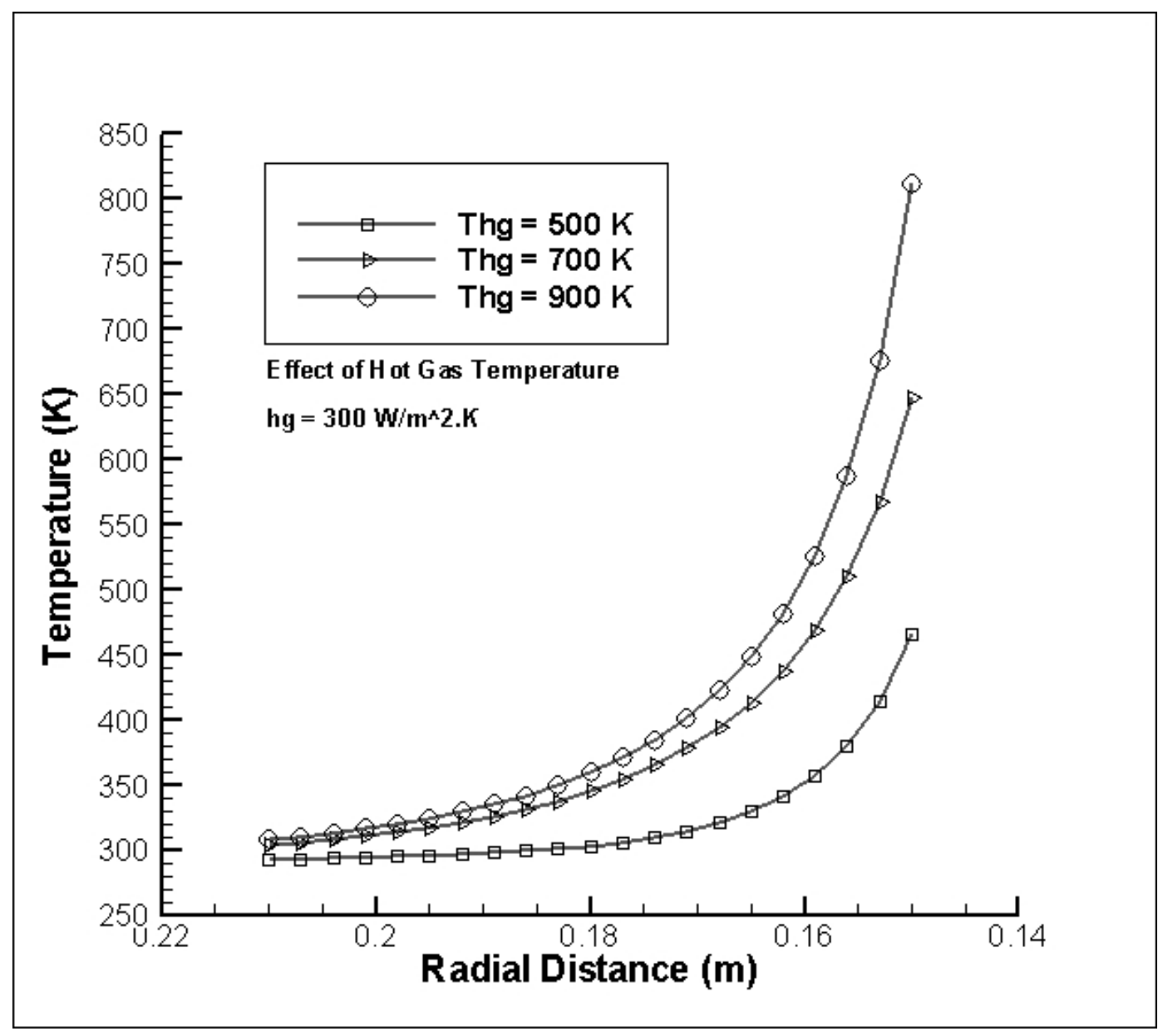

Figure 26: Effect of hot gas temperature on temperature profile within the sample.

\subsection{Effect of Coolant Gas Type}

Transpiration cooling problem was previously solved using Hydrogen as the coolant. In this part Air and Helium are tested to compare their cooling capacities with that of Hydrogen. These gases have different specific heats and thermal conductivities, hence, different cooling effects. It is assumed that a constant heat flux incoming to the sample at the hot face of $5000 \mathrm{~W} / \mathrm{m}^{2}$, and the mass flow rate of the coolants are taken $7.0 \mathrm{gr} / \mathrm{s}$. The porous material is assumed to be Si-C with the specified properties. Corresponding temperature distributions in the sample are obtained and shown in Fig.26. 


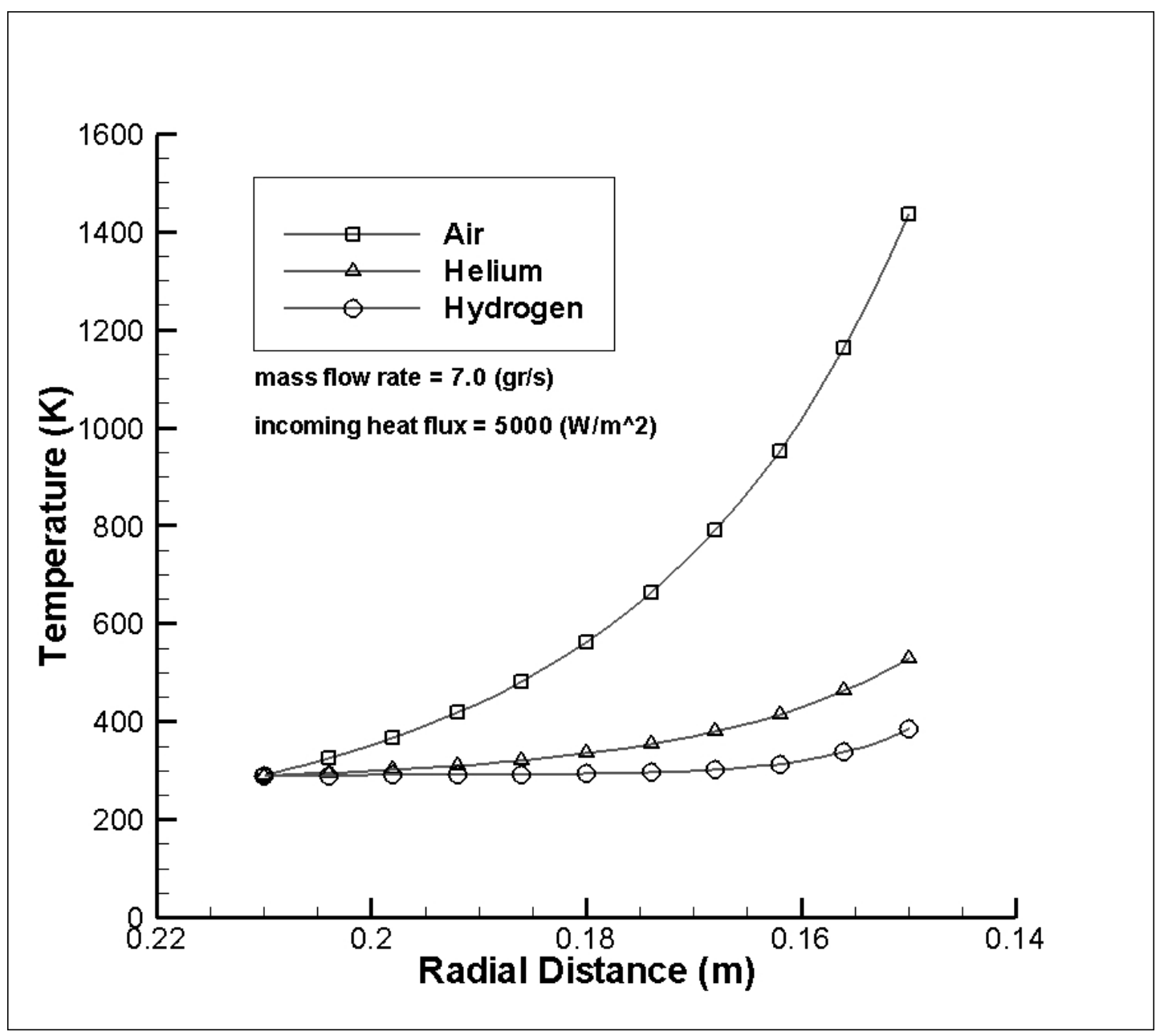

Figure 27: Temperature profile within the sample for different gases: Air, Helium, and Hydrogen.

According to this figure, the hot face temperature of Air reaches $1436 \mathrm{~K}$, which is beyond the temperatures of the other gases. Helium and Hydrogen yield 529.3 and 384.6 K respectively at the hot face of the sample. The cooling capacity of Hydrogen is observed to be higher than Helium and Air, implying that Hydrogen is the best coolant among these gases. The reason is the very high heat capacity of Hydrogen as well as its high thermal conductivity which can absorb and transfer the heat along the coolant flow. Moreover, the cooling capacity of Air is less than the other two gases, which make this gas a weak choice for using as the coolant. 


\subsection{Effect of Sample Thickness}

Another parameter is the sample length (radial thickness) which has a significant effect on transpiration cooling. Similar Si-C porous material is selected with different lengths of $6,8,12,16$, and $20 \mathrm{~cm}$. Incoming heat flux of $5000 \mathrm{~W} / \mathrm{m}^{2}$ and mass flow rate of $2 \mathrm{gr} / \mathrm{s}$ are applied to the problem. Temperature profiles are then compared and shown in Fig.27.

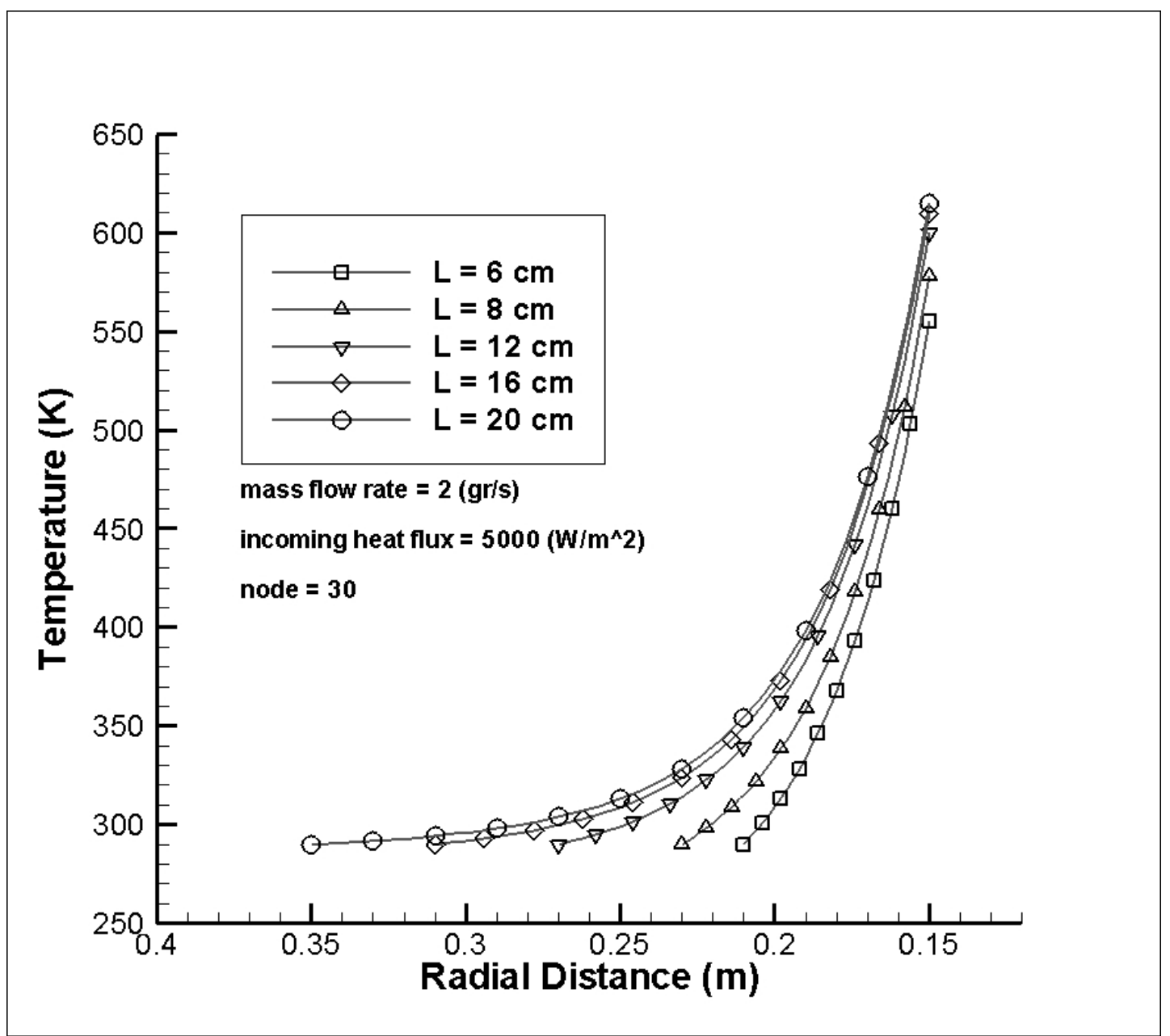

Figure 28: Temperature profile within the sample for different sample lengths of 6,8 , 12,16 , and $20 \mathrm{~cm}$.

According to this figure, the hot face temperature increases as the length increases. The obtained hot temperatures for the specified lengths are 555.01, 577.64, 600.14, 
609.85, and 614.8 K accordingly. As the length of the sample increases, the diffusive heat transfer toward the cold face decreases, which causes more heat storage within the sample and, therefore, higher temperature at each node.

\subsection{Hot Gas Flow}

In the previous section, a comprehensive analysis of the parameters in the coolant flow through the porous media was performed. However, it is more appropriate that these factors are shown in a more realistic condition occurred with the transpiration cooling. Therefore, in this part, transpiration cooling is studied, concerning its nature of blocking and carrying the incoming heat back into the hot gas stream. To achieve this goal, the equations of flow within the porous medium are solved coupled with the equations of hot gas in the thrust chamber. The nozzle profile explained in the section 4 is assumed to be in contact with a porous segment. The porous segment can be assumed as a hollow cylinder which covers the converging part of the nozzle until its throat. Therefore, it has a length of about $0.5 \mathrm{~m}$, which is the length of the converging part of the nozzle. The segment is made of Si-C porous material and has a uniform thickness of $6 \mathrm{~cm}$. The segment is assumed to be in the way that the radial channels are manufactured and attached together, through which, the coolant is injected towards the nozzle. That is, the segment can be considered as onedimensional cylindrical channels which are totally covering the converging part of the nozzle (see Fig.28). 


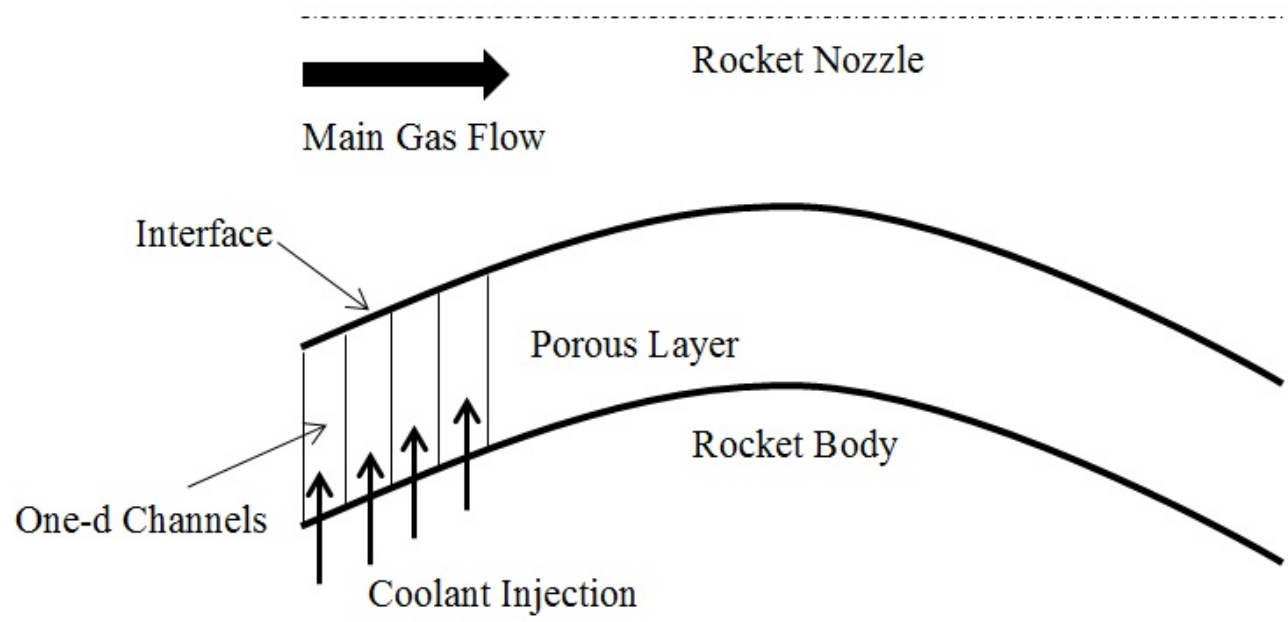

Figure 29: Porous one-d segments covering the converging part of nozzle.

The advantage of this model is that the one-dimensional equations of transpiration cooling (i.e. continuity, momentum, and energy) are applicable within the channels. According to the explained configuration, the coolant fluid has only radial paths to march to the nozzle wall. But, the conductive heat transfer within the segment can be in both radial and axial directions. However, due to the focus on the convective effect within the porous channels, special manufacturing can be carried out such that the axial term of the conductive heat transfer can be assumed to be negligible.

As mentioned above, the case is defined a set of a converging-diverging nozzle and a porous segment, covering the converging part of it. The coolant is assumed to be liquid Hydrogen which is the fuel of the liquid rocket and therefore, the combustion gas products (i.e. water vapor) are flowing through the nozzle. The inlet condition of the nozzle is assumed to be $\mathrm{M}_{\mathrm{i}}=0.301, \mathrm{~T}_{\mathrm{i}}=986.7 \mathrm{~K}, \mathrm{P}_{\mathrm{i}}=9.44 \mathrm{e} 5 \mathrm{~Pa}$.

The porous segment is a Si-C material with the given properties in the previous section. Also, the coolant has the initial pressure and temperature of $1.35 \mathrm{MPa}$ and 
$290 \mathrm{~K}$, respectively. The case can be set as controlling the temperature of the nozzle wall by means of transpiration cooling. Setting a limiting temperature, the solution procedure is as following

A - Solve the equation of hot gas for an isentropic flow (i.e. Eqs.39 and 40).

B - According to the Mach number profile, the velocity profile and then, the heat transfer coefficient can be calculated (i.e. Eq.29).

$\mathrm{C}$ - The gas temperature distribution obtain in the previous section is applied as the hot gas temperature (i.e. Eq.42).

$\mathrm{D}$ - The porous flow equations in all the channels are solved with an initial value of coolant mass flow rate (i.e. Eqs.1 to 3).

E - The wall temperatures obtained section D is compared with the allowed temperature.

F - If the wall temperature of a channel exceeds the allowed temperature, the coolant flow rate is raised.

$\mathrm{G}$ - The gas equations are solved with the new condition of coolant mass addition and the heat convection absorbed at the walls (i.e. Eqs.39 and 40).

$\mathrm{H}$ - Go to section B and continue the loop until the wall temperature reaches the desirable value.

The limiting temperature for this case is set to $750 \mathrm{~K}$. The results are obtained and demonstrated in the following figures. The results in the nozzle are shown comparing the initial solution (i.e. isentropic flow) and the final solution. 


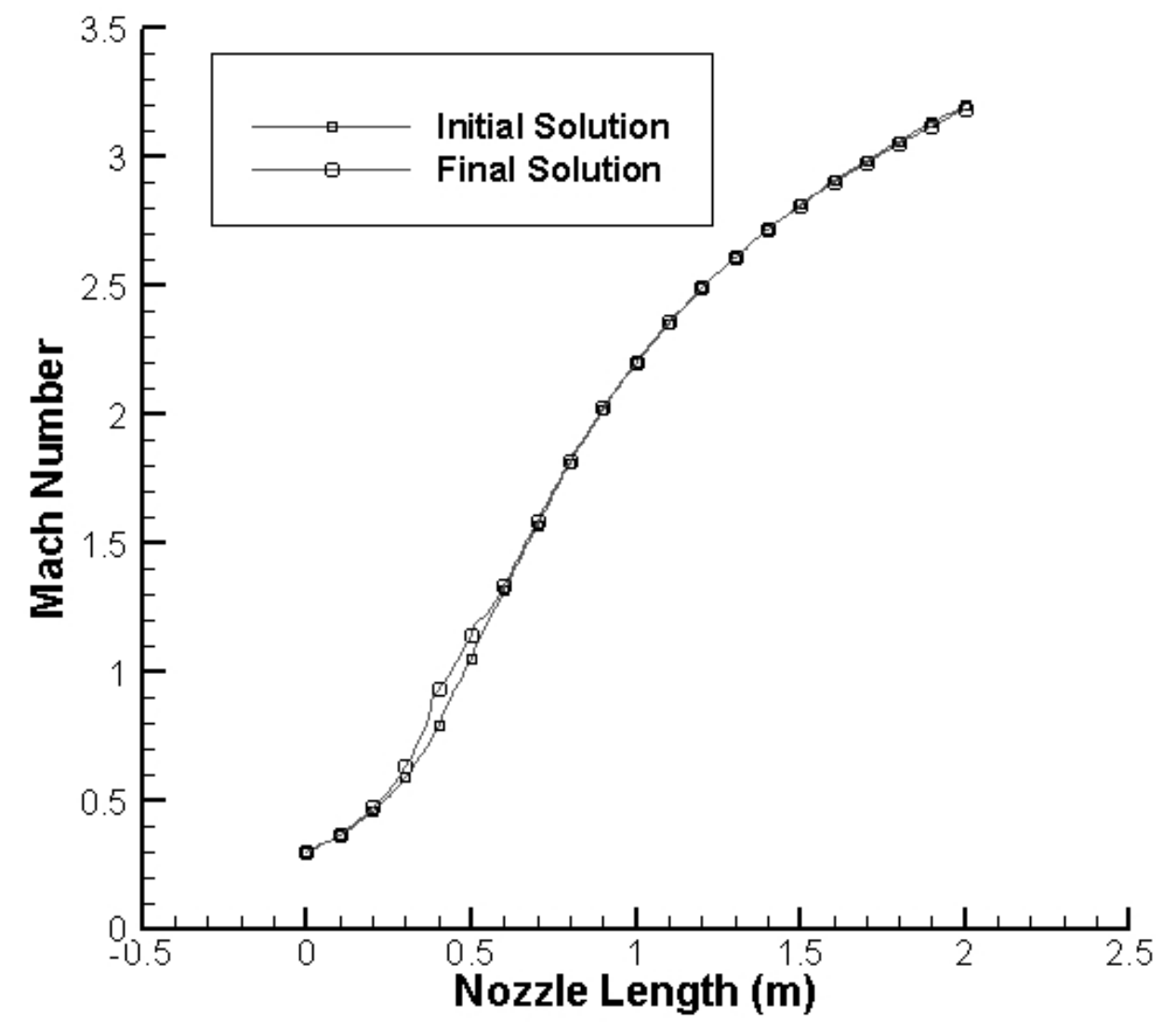

Figure 30: Mach number profile within the nozzle for initial and final solutions.

Figure.29 demonstrates the Mach number distribution. As to the isentropic flow, the Mach number reaches to one at the throat and then, increases to a value of three at the exit. It can be observed that the Mach number distribution does not change significantly. This means that the flow conditions in the converging, throat, and diverging sections maintain the standard working conditions. Hot gas temperature distribution is given in Fig.30. Unlike the Mach number, temperature of the gas shows an increase within the nozzle. In the isentropic flow, the gas temperature is about $980 \mathrm{~K}$ at the inlet, and due to the condition of flow, it decreases along the nozzle to $400 \mathrm{~K}$ at the outlet. After transpiration cooling, the temperature increases locally in the converging part on the nozzle and obtains a value of $994 \mathrm{~K}$, afterwards 
it decreases as it flows through the nozzle. Figure.30 shows that the transpiration cooling process shifts the temperature profile upward. This increase can be due to the heat blocking effect if the transpiration cooling.

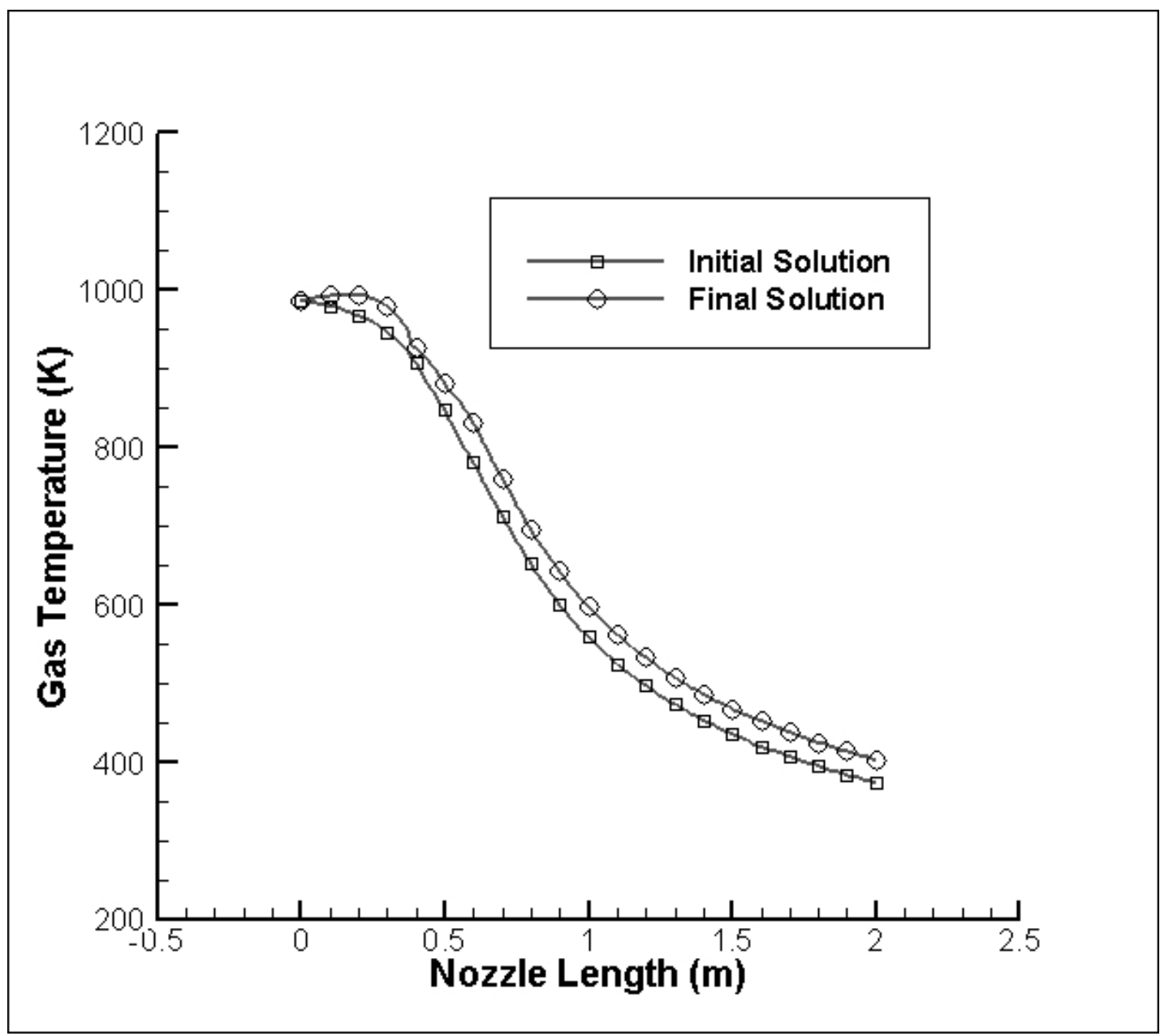

Figure 31: Gas temperature profile within the nozzle for initial and final solutions.

Also, the coefficient of heat transfer at the nozzle wall is illustrated in Fig.31. The coefficient of heat transfer is obtained from the state of heat balance at the hot face as $\left.-k \frac{\partial T}{\partial r}\right]_{\text {at hot face }}=h\left(T_{\text {wall }}-T_{\text {gas }}\right)$

The profile starts at about $600 \mathrm{~W} / \mathrm{m}^{2} . \mathrm{K}$ at the inlet and reaches to $1350 \mathrm{~W} / \mathrm{m}^{2} . \mathrm{K}$ at the throat. 


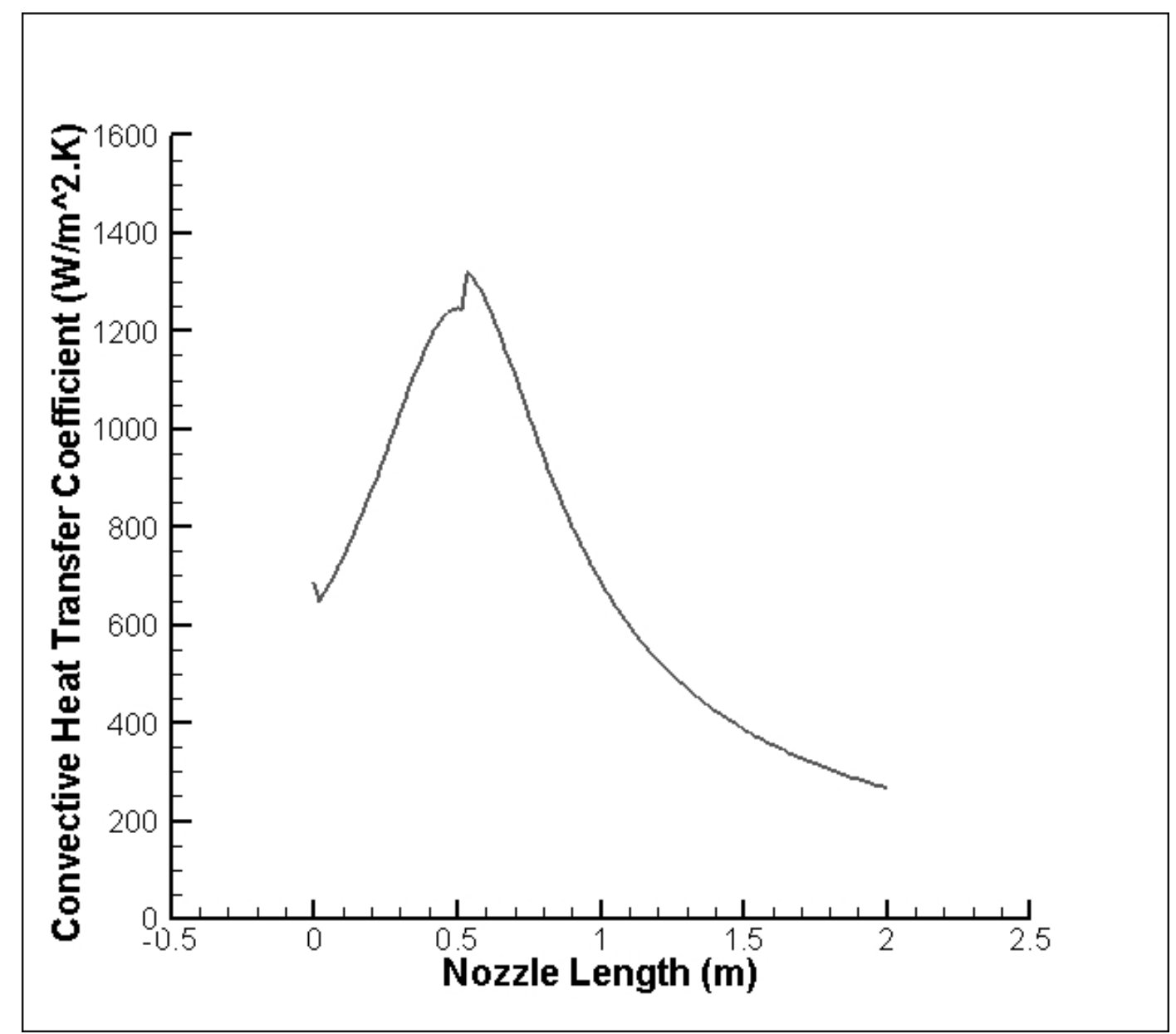

Figure 32: Coefficient of convective heat transfer at the wall for the final solution.

This profile has a maximum about the location of the throat. After the throat, the profile declines along the nozzle to the value of $300 \mathrm{~W} / \mathrm{m}^{2} . \mathrm{K}$ at the exit. The reason is that the coefficient of heat transfer is function of both velocity and temperature of the gas. Therefore, the coefficient is higher at the throat compare to the other points of the nozzle. Then, the convective heat transfer between the hot gases and the nozzle is also calculated and presented in Fig.32. 


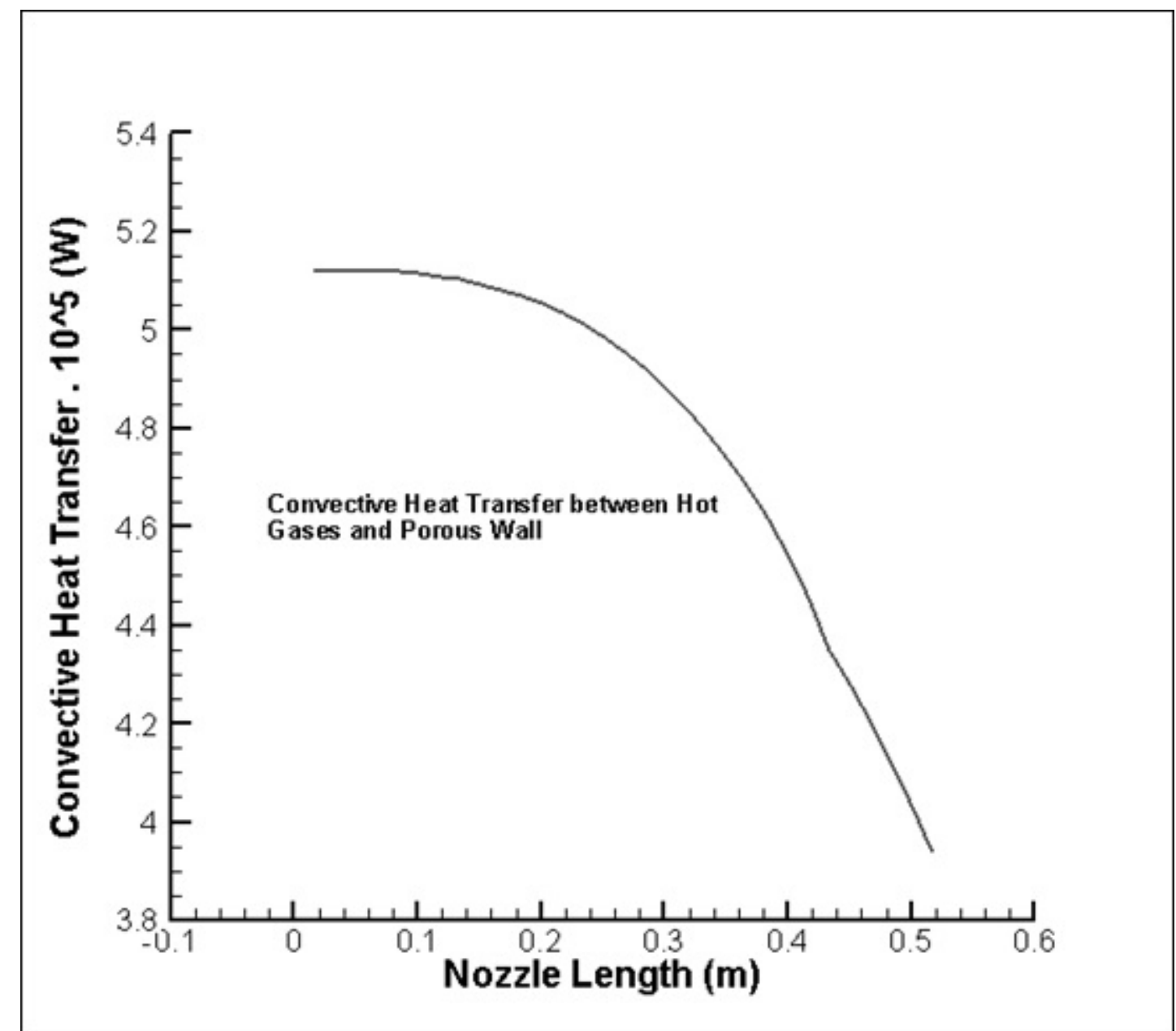

Figure 33: Convective heat transfer profile between the hot gases and the porous wall within the nozzle.

This figure shows that the convection has its maximum of 5.15e5 $\mathrm{W}$ at the beginning of the nozzle, and it goes down to $4 \mathrm{e} 5 \mathrm{~W}$ at the throat. Despite of this difference, the range of the heat transfer is at a similar order. The total mass flow rate of the coolant along the porous segment is calculated to be $6.0 \mathrm{~kg} / \mathrm{s}$. Also, the average mass flow rate of the coolant from each channel is about $0.2 \mathrm{~kg} / \mathrm{s}$. The distribution of the coolant mass flow rate is almost uniform. The reason is that at the beginning of the nozzle the gas temperature is higher than the other points, and at the throat the coefficient of heat transfer is higher than the other points. Therefore, both regions create an almost similar hot face condition at the nozzle walls. Finally, the hot face 
temperature, which is controlled by the coolant flow though the porous channels, is demonstrated in Fig.33. It shows the hot face temperatures at the beginning of the solution (i.e. initial solution) until the model can control the hot face temperature by transpiration cooling (i.e. final solution). All the values are below $750 \mathrm{~K}$, as presented in this figure. Also, the curve shows that the transpiration cooling is more effective at the beginning and the throat of the nozzle compare to the other points.

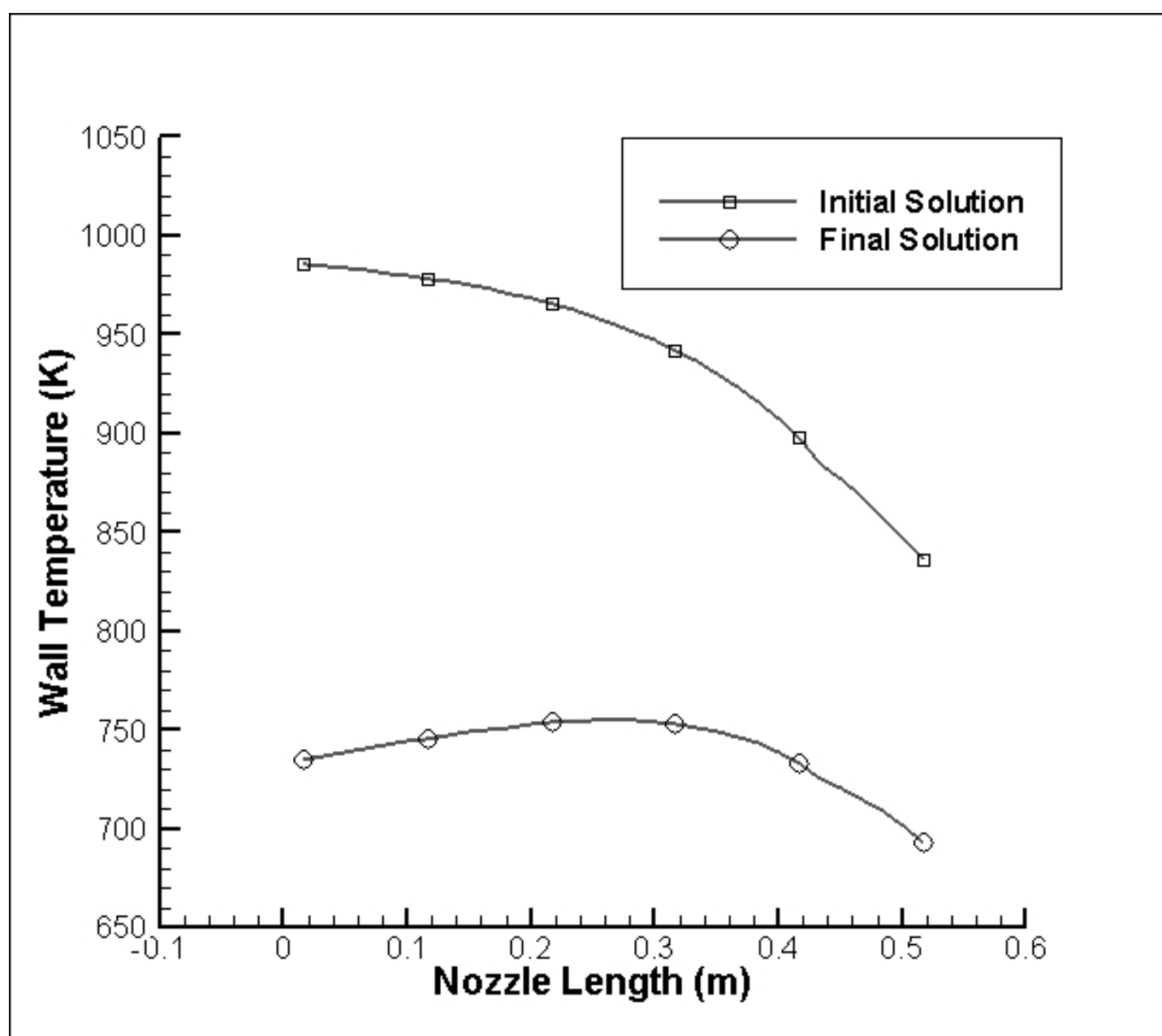

Figure 34: Wall temperature distribution within the nozzle for the initial and final solutions. 


\section{Chapter 7}

\section{CONCLUSION AND FUTURE WORK}

In the current study, steady state one-dimensional transpiration cooling in cylindrical coordinates was modeled as a combination of engineering parametric study and realistic situations and boundary conditions. The obtained numerical results were validated with experimental results obtained from a $\mathrm{PhD}$ dissertation at Stuttgart University. The numerical results showed good accordance with the experimental results and could be validated very well. Mathematical modeling of flow within the porous media in cylindrical coordinates was also coupled with a quasi-twodimensional simulation of gas flow in the thrust chamber. The numerical investigation demonstrates that the finite difference method presented in this research is appropriate for modeling flow in porous media. Detailed temperature distribution of flow in the porous material can be obtained from the numerical results, as it is difficult to gain them from experimental results because of its limitations. It will cause difficulties to place measuring devices in a porous material at such small distances. Although good agreement has obtained with the experimental results, numerical results are strongly dependent on some parameters like heat transfer coefficient at the cold side which need to be determined from experimental data. However, assuming thermal equilibrium between solid and fluid does not yield much error, especially for higher coolant mass flow rates. 
The porous media model has main assumptions of one-dimensional cylindrical geometry, Darcian velocity conditions and thermal equilibrium between solid and fluid. Also, the injected coolant mass flow rate is to be prescribed. Then, the results of fluid density and velocity within the porous material can be interpreted. At the coolant entrance area and the beginning of the porous material, the fluid is at higher pressure and lower velocity. At the end of porous material, where the coolant is exiting, the velocity rises as result of two factors, first, decreasing density due to increasing temperature and getting close to the hot face, and last, decreasing cross sectional area of the porous material. Also, it can be concluded that this velocity rise, is more effective for higher coolant mass flow rates.

Moreover, effect of hot face conditions was investigated. It is shown as hot gas temperature and heat transfer coefficient change changes. The coefficient of heat transfer at the hot face is a affected by the main flow properties such as flow velocity (Mach number), local temperature, and some thermal properties. This comparison demonstrates that heat transfer coefficient has greater effect on incoming heat to the porous material. Therefore, controlling the main flow properties can help to reduce the incoming heat flux to the walls. Also, heat flux analysis was carried out in order to observe the total amount of the heat transfer which can be blocked through the porous medium towards the thrust chamber walls. In addition, a dimensionless parameter called cooling efficiency was defined and calculated, as it can better represent the effect of transpiration cooling on the temperature profile and heat flux in the porous material. It increases rapidly as the coolant flow rate increases, and seems to stabilize within an interval which can be defined as the maximum cooling efficiency. 
Then, parametric studies were carried out to observe the effect of gas type and sample thickness on the cooling process. Hydrogen showed a very perfect gas as the coolant while air could not stand as a proper cooling fluid. The effect of length change was also studied on the cooling. It was shown that the increase in the sample thickness increases the heat blocking effect and magnifies the cooling process, though with greater temperature values.

Coupling of porous medium flow to the fluid flow is a field of research which lacks in both theoretical investigations and practical application of numerical tools, that is, there has been no approach of performing a fully coupled simulation of transpiration cooling so far [19]. The problem was defined such that a porous segment surrounded the converging part of the nozzle, which has the highest heating load of the nozzle. In this case, two codes needed to be coupled and then solved alternatively. During this modeling, more parameters, such as heat transfer coefficient along the nozzle, convective heat transfer between the gas and the porous wall, and the porous wall temperature were obtained and studied. Also, the total and averaged coolant mass flow rate, and the effect of transpiration cooling on the nozzle flow properties, such as Mach number and gas temperature were obtained and demonstrated. The distribution of coefficient of heat transfer along the nozzle demonstrated that the major heat transfer to the thrust chamber wall occurs in the converging part of the nozzle, where the flow is subsonic, until the throat.

The numerical study of the transpiration cooling is an ongoing area of investigation. One of the primary assumptions in the modeling in this research was the illumination of coolant evaporation within the porous material. In reality, at the coolant entrance, 
there is evaporation occurring, and the latent heat and proper equations for two phases need to be assumed.

It is also recommended that a two-dimensional cylindrical transpiration cooling model is investigated to observe the axial temperature gradient and coolant flow within the porous material. This model, as the cooling part of a rocket engine, in order to obtain the optimum performance of the engine, needs further research and inquiry. In the limited experimental access to the subtle processes occurring at the interface between hot gas flow and porous medium, and also measuring device, reliable and accurate simulations become an increasingly important design tool. 


\section{REFERENCES}

[1] Kays W.M., Crawford M.E. and Weigand B., Convective heat and mass transfer. 4th Edition ed. 1993: McGraw-Hill, Inc., New York.

[2] Rannie W., A simplified theory of porous wall cooling. 1947, Technical Report, JPL California Institute of Technology: Pasadena, USA.

[3] Eckert E. and Livingood N., Comparison of effectiveness of convection-, transpiration-, and film-cooling methods with air as coolant. 1954, NACA: Technical Report, Lewis Flight Propulsion Laboratory,Cleveland, OH.

[4] Glass D.E., Dilley A.D., and H.N. Kelly, Numerical analysis of convection/transpiration cooling. Journal of Spacecraft and Rockets, 2001. 38(1): p. 15-20.

[5] Meinert Jens, Meinert, J-ograve, Huhn rg, Serbest Erhan, Haidn Oskar J., Turbulent boundary layers with foreign gas transpiration. Journal of Spacecraft and Rockets, 2001. 38(2): p. 191-198.

[6] Mathelin L., Bataille F., and Lallemand A., Blowing models for cooling surfaces. International journal of thermal sciences, 2001. 40(11): p. 969-980.

[7] Tully L.R., Numerical Modeling of Transpiration Cooled Rocket Injectors. 2005, University of Florida. 
[8] Lezuo M. and Haidn O.J, Transpiration cooling using gaseous hydrogen. in 33rd joint propulsion conference and exhibit. 1997.

[9] Hannemann V., Numerical investigation of an effusion cooled thermal protection material, in Computational Fluid Dynamics 2006. 2009, Springer. p. 671-676.

[10] Wang J. and Wang H., A discussion of transpiration cooling problems through an analytical solution of local thermal nonequilibrium model. Journal of heat transfer, 2006. 128(10): p. 1093-1098.

[11] Greuel D., Herbertz Armin, Haidn OJ, Ortelt Markus and Hald Hermann. Transpiration cooling applied to C/C liners of cryogenic liquid rocket engines. in 40th Joint Propulsion Conference. 2004. Capua, Italy: AIAA 2005-3229.

[12] Steelant J., ATLLAS: Aero-thermal loaded material investigations for highspeed vehicles, in 15th AIAA International Space Planes and Hypersonic Systems and Technologies Conference. 2008: Dayton, OH.

[13] Sözen M. and Davis P.A., Transpiration Cooling of a Liquid Rocket Thrust Chamber Wall. AIAA Paper, 2008. 4559: p. 2008.

[14] Cheuret F., Steelant Johan, Langener T, Von Wolfersdorf J. Simulations of Transpiration Cooling for Supersonic Flow. in Proceedings of the CEAS 2009 European Air and Space Conference. 2009. Manchester, UK. 
[15] Langener T., A contribution to transpiration cooling for aerospace applications using CMC walls, in PhD Thesis, Institute of Aerospace Thermodynamics. University of Stuttgart, 2011.

[16] Langener T., Wolfersdorf J.V. and Steelant J., Experimental investigations on transpiration cooling for scramjet applications using different coolants. AIAA journal, 2011. 49(7): p. 1409-1419.

[17] Gascoin N., Romagnosi Luigi, Fedioun Ivan, Fau Guillaume, Gillard Philippe, Steelant Johan. Pyrolysis in Porous Media: Numerical Analysis and Comparison to Experiments. in 18th AIAA/3AF International Space Planes and Hypersonic Systems and Technologies Conference. 2012.

[18] Romagnosi L., Gascoin Nicolas, El-Tabach Eddy, Fedioun Ivan, Bouchez Marc, Steelant, Johan, Pyrolysis in porous media: Part 1. Numerical model and parametric study. Energy Conversion and Management, 2013. 68: p. 6373.

[19] Dahmen W., Gotzen Thomas, Müller Siegfried, Rom M., Numerical simulation of transpiration cooling through porous material. International Journal for Numerical Methods in Fluids, 2014. 76(6): p. 331-365.

[20] Dahmen W., Gotzen Thomas, Melian Sorana, Müller Siegfried, Numerical simulation of cooling gas injection using adaptive multiresolution techniques. Computers \& Fluids, 2013. 71: p. 65-82. 
[21] Alazmi B. and Vafai K., Analysis of fluid flow and heat transfer interfacial conditions between a porous medium and a fluid layer. International Journal of Heat and Mass Transfer, 2001. 44(9): p. 1735-1749.

[22] Ferziger J.H. and Perić M., Computational methods for fluid dynamics. 1996: Springer, Inc., New York.

[23] Cengel Y.A., Boles M.A. and Kanoglu M., Thermodynamics: an engineering approach. Vol. 1056. 1998: McGraw-Hill New York.

[24] Çengel Y.A. and Ghajar A.J., Heat and mass transfer: fundamentals \& applications. 2011: McGraw-Hill.

[25] Patankar S., Numerical heat transfer and fluid flow. 1980: CRC Press, NY, US.

[26] Dittus F. and Boelter L., Heat transfer in automobile radiators of the tubular type. International Communications in Heat and Mass Transfer, 1985. 12(1): p. 3-22.

[27] Chi S., Heat pipe theory and practice: a sourcebook. 1976.

[28] Zucrow M.J. and Hoffman J.D., Gas dynamics. Volume 1. New York, John Wiley and Sons, Inc., 1976. 1.

[29] John J. and Keith T., Gas dynamics. 1984. 
[30] Dekker K. and Verwer J.G., Stability of Runge-Kutta methods for stiff nonlinear differential equations. 1984. 